\title{
Supporting Information for \\ Discovery of the First Potent IDO1/IDO2 Dual Inhibitors: A Promising Strategy for Cancer Immunotherapy
}

Xin $\mathrm{He}^{\mathrm{a}, \S}$, Guangchao $\mathrm{He}^{\mathrm{a}, \S}$, Zhaoxing $\mathrm{Chu}^{\mathrm{b}}$, Huanhuan $\mathrm{Wu}^{\mathrm{b}}$, Junjie Wang ${ }^{\mathrm{b}}$, Yiran $\mathrm{Ge}^{\mathrm{b}}$, Hui Shen ${ }^{\mathrm{b}}$, Shan Zhang ${ }^{\mathrm{b}}$, Jinxi Shan ${ }^{\mathrm{b}}$, Kewen Peng ${ }^{\mathrm{b}}$, Zhifeng Weic, Yi Zou ${ }^{\mathrm{a}}$, Yungen $\mathrm{Xu}^{\mathrm{a}, \mathrm{b},{ }^{*}}$ and Qihua $\mathrm{Zhu}^{\mathrm{a}, \mathrm{b},{ }^{*}}$

${ }^{a}$ State Key Laboratory of Natural Medicines, China Pharmaceutical University, Nanjing, 210009, China

${ }^{b}$ Jiangsu Key Laboratory of Drug Design and Optimization, Department of Medicinal Chemistry, China Pharmaceutical University, Nanjing 211198, China

${ }^{c}$ Department of Pharmacology of Chinese Materia Medica, School of Traditional Chinese Pharmacy, China Pharmaceutical University, 24 Tong Jia Xiang, Nanjing 210009, China

\section{Corresponding Authors}

Yungen Xu, Email: xyg@cpu.edu.cn

Qihua Zhu, Email: zhuqihua@vip.126.com

\section{Contents}

1. Homology modeling of 3D structure of IDO2 protein...........................S3

2. The dose-response curves of compounds in enzyme assay......................S4

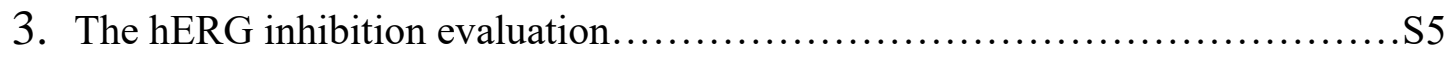

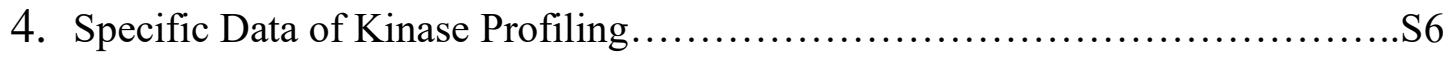

5. Cell-based assay of IDO1 inhibitors in HeLa cell..............................S15

6. Antitumor activity assay of candidate in vivo................................... 15

7. ${ }^{1} \mathrm{H}$ NMR and ${ }^{13} \mathrm{C}$ NMR Spectra of All Target Compounds.......................... 16

8. HRMS Spectra of All Target Compounds................................... 38 


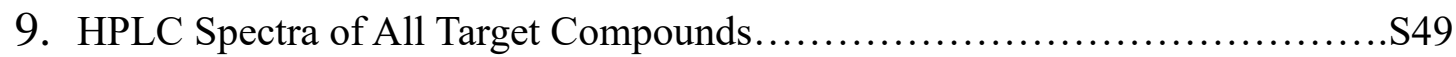


1. Homology modeling of $3 \mathrm{D}$ structure of IDO2 protein

Firstly, module protein was obtained by sequence alignment of IDO2 and IDO1 followed by search the IDO1 complex (PDB code: 5WN8) (Figure S1 A). Secondly, modeler 9.17 (Sali, A.; Blundell, T. L. Comparative protein modeling by satisfaction of spatial restraints. J. Mol. Biol. 1993, 234, 779-815.) was utilized to homology modeling, two consensus models feathered with max GA341, min molpdf and DOPE score, were used as the candidate 3D structures of IDO2 (Figure S1 B).

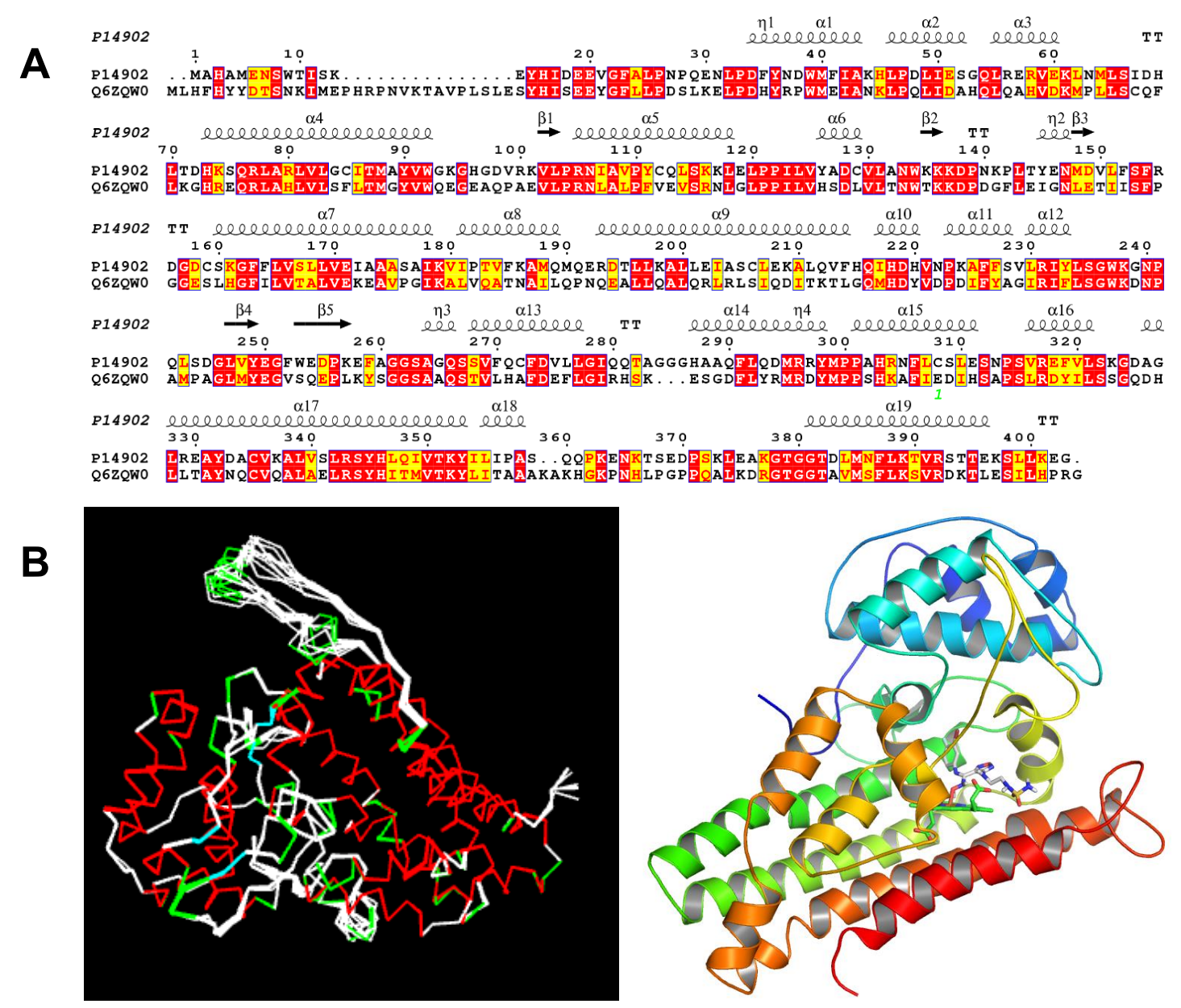

Figure S1 (A) Sequence alignments of IDO1 and IDO2 (B) IDO2 homology model based on a holo X-ray

structure of the IDO1 
2. The dose-response curves of compounds in enzyme assay
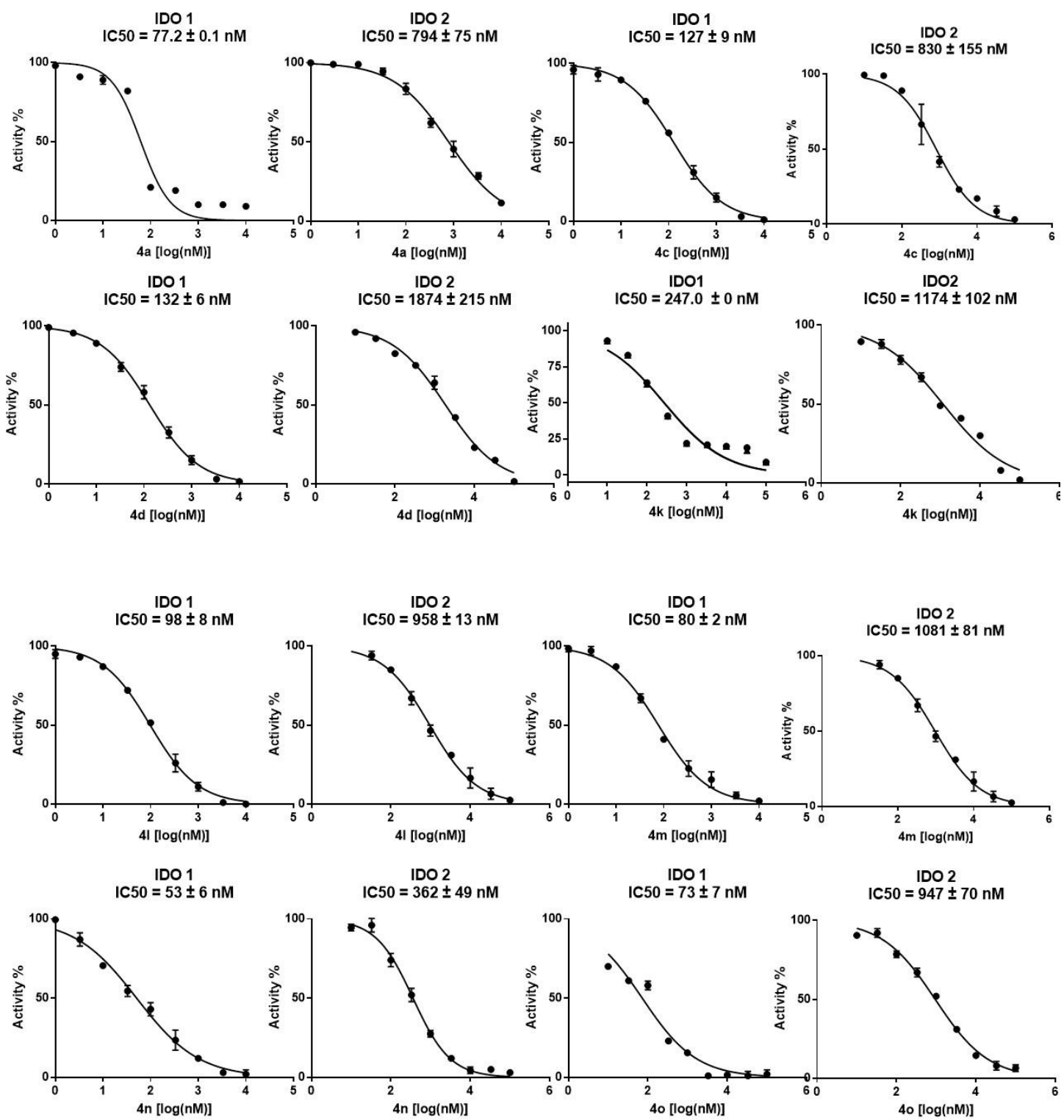

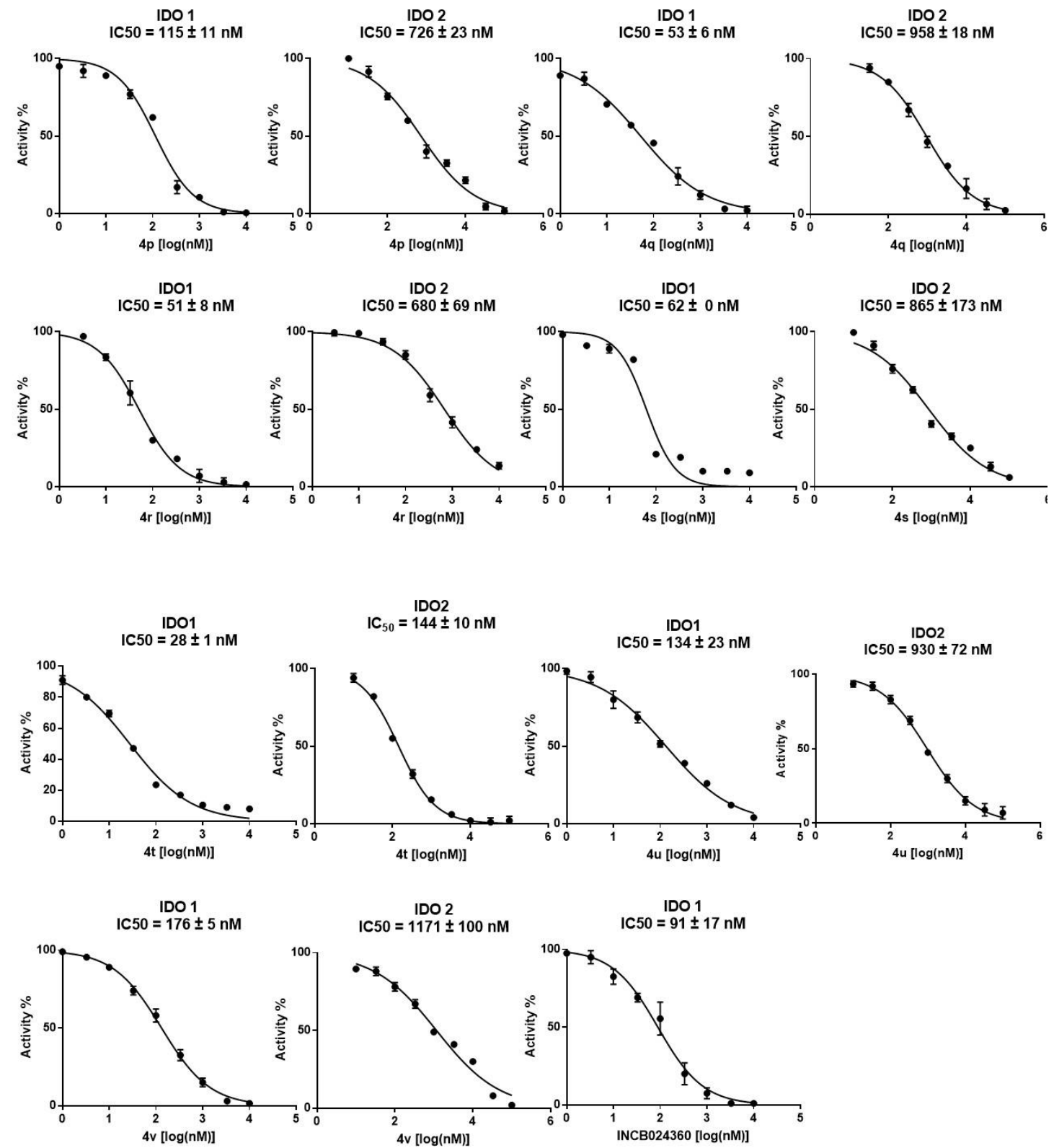

Figure S2 The dose-response curves of representative compounds in enzyme assay

\section{The evaluation of cardiotoxicity assay}

Table S1 The hERG inhibition of compounds $4 \mathrm{n}$ and $4 \mathrm{t}$

\begin{tabular}{|c|c|c|c|c|c|c|c|}
\hline \multirow{2}{*}{ Compd. } & \multicolumn{5}{|c|}{ hERG inhibition (\%) } & \multirow{2}{*}{$\mathrm{n}$} & \multirow{2}{*}{$\begin{array}{l}\mathrm{IC}_{50} \\
(\mu \mathrm{M})\end{array}$} \\
\hline & $0.3 \mu \mathrm{M}$ & $1 \mu \mathrm{M}$ & $3 \mu \mathrm{M}$ & $10 \mu \mathrm{M}$ & $30 \mu \mathrm{M}$ & & \\
\hline $4 n$ & $0.64 \pm 0.30$ & $4.82 \pm 2.00$ & $10.13 \pm 0.45$ & $18.03 \pm 1.85$ & $40.09 \pm 3.12$ & 3 & $>30$ \\
\hline $4 \mathrm{t}$ & $-4.92 \pm 1.37$ & $1.05 \pm 0.40$ & $7.12 \pm 0.96$ & $9.89 \pm 0.80$ & $-12.12 \pm 2.35$ & 3 & $>30$ \\
\hline
\end{tabular}




\section{Specific Data of Kinase Profiling}

Table S2. Specific Data of Kinase Profiling of Compound 4t

\begin{tabular}{|c|c|c|c|c|}
\hline \multirow{3}{*}{ Kinase } & $\begin{array}{l}\text { Enzyr } \\
\text { (relative }\end{array}$ & $\begin{array}{l}\text { ty }(\%) \\
\text { controls) }\end{array}$ & \multirow{3}{*}{$\begin{array}{l}\text { Control Compd } \\
\mathrm{IC}_{50}(\mathrm{M})\end{array}$} & \multirow{3}{*}{$\begin{array}{l}\text { Control Compd } \\
\text { ID }\end{array}$} \\
\hline & \multicolumn{2}{|c|}{$4 \mathrm{t}(1 \mu \mathrm{M})$} & & \\
\hline & Data 1 & Data 2 & & \\
\hline ABL1 & 98.88 & 93.59 & $6.13 \mathrm{E}-08$ & Staurosporine \\
\hline ABL2/ARG & 102.23 & 101.99 & $1.77 \mathrm{E}-08$ & Staurosporine \\
\hline ACK1 & 104.85 & 101.95 & $2.13 \mathrm{E}-08$ & Staurosporine \\
\hline AKT1 & 111.02 & 109.87 & 7.30E-09 & Staurosporine \\
\hline AKT2 & 95.38 & 93.47 & 7.90E-09 & Staurosporine \\
\hline AKT3 & 84.74 & 84.10 & $2.11 \mathrm{E}-09$ & Staurosporine \\
\hline ALK & 88.45 & 87.21 & $1.35 \mathrm{E}-09$ & Staurosporine \\
\hline ALK1/ACVRL1 & 90.58 & 89.67 & $1.63 \mathrm{E}-08$ & LDN193189 \\
\hline ALK2/ACVR1 & 102.56 & 101.37 & 2.01E-08 & LDN193189 \\
\hline ALK3/BMPR1A & 106.25 & 104.41 & $3.72 \mathrm{E}-08$ & LDN193189 \\
\hline ALK4/ACVR1B & 97.95 & 95.52 & $2.57 \mathrm{E}-07$ & LDN193189 \\
\hline ALK5/TGFBR1 & 101.09 & 101.04 & $3.81 \mathrm{E}-07$ & LDN193189 \\
\hline ALK6/BMPR1B & 95.06 & 93.63 & $1.60 \mathrm{E}-08$ & LDN193189 \\
\hline ARAF & 97.28 & 95.53 & $2.48 \mathrm{E}-08$ & GW5074 \\
\hline ARK5/NUAK1 & 108.54 & 108.26 & $5.92 \mathrm{E}-10$ & Staurosporine \\
\hline ASK1/MAP3K5 & 120.69 & 116.15 & $2.67 \mathrm{E}-08$ & Staurosporine \\
\hline Aurora A & 93.00 & 92.18 & $9.76 \mathrm{E}-10$ & Staurosporine \\
\hline Aurora B & 99.81 & 99.37 & $6.84 \mathrm{E}-09$ & Staurosporine \\
\hline Aurora C & 87.33 & 86.20 & 4.20E-09 & Staurosporine \\
\hline AXL & 82.14 & 81.85 & $3.20 \mathrm{E}-09$ & Staurosporine \\
\hline BLK & 99.68 & 99.02 & $1.47 \mathrm{E}-09$ & Staurosporine \\
\hline BMPR2 & 94.51 & 93.49 & $3.29 \mathrm{E}-07$ & Staurosporine \\
\hline BMX/ETK & 96.43 & 93.92 & $5.86 \mathrm{E}-09$ & Staurosporine \\
\hline BRAF & 97.67 & 97.34 & 4.32E-08 & GW5074 \\
\hline BRK & 100.83 & 100.67 & $2.38 \mathrm{E}-07$ & Staurosporine \\
\hline BRSK1 & 87.30 & 85.76 & 4.15E-10 & Staurosporine \\
\hline BRSK2 & 85.95 & 85.73 & $1.68 \mathrm{E}-09$ & Staurosporine \\
\hline BTK & 86.79 & 84.91 & $2.05 \mathrm{E}-08$ & Staurosporine \\
\hline c-Kit & 82.21 & 82.20 & $6.17 \mathrm{E}-08$ & Staurosporine \\
\hline c-MER & 97.90 & 97.26 & $2.05 \mathrm{E}-08$ & Staurosporine \\
\hline c-MET & 82.85 & 82.17 & $1.74 \mathrm{E}-07$ & Staurosporine \\
\hline $\mathrm{c}-\mathrm{Src}$ & 93.60 & 92.96 & $1.91 \mathrm{E}-09$ & Staurosporine \\
\hline CAMK1a & 98.72 & 97.70 & 4.88E-09 & Staurosporine \\
\hline CAMK1b & 94.27 & 92.29 & $8.12 \mathrm{E}-09$ & Staurosporine \\
\hline
\end{tabular}




\begin{tabular}{|c|c|c|c|c|}
\hline CAMK1d & 94.26 & 93.28 & 4.61E-10 & Staurosporine \\
\hline CAMK1g & 93.89 & 89.48 & $5.55 \mathrm{E}-09$ & Staurosporine \\
\hline CAMK2a & 96.67 & 96.23 & $2.32 \mathrm{E}-11$ & Staurosporine \\
\hline CAMK2b & 95.74 & 93.50 & $5.96 \mathrm{E}-11$ & Staurosporine \\
\hline CAMK2d & 96.59 & 94.78 & $5.26 \mathrm{E}-11$ & Staurosporine \\
\hline CAMK2g & 89.06 & 81.10 & $5.31 \mathrm{E}-10$ & Staurosporine \\
\hline CAMK4 & 101.61 & 101.42 & $1.50 \mathrm{E}-07$ & Staurosporine \\
\hline CAMKK1 & 93.81 & 93.31 & $6.66 \mathrm{E}-08$ & Staurosporine \\
\hline CAMKK2 & 85.87 & 79.17 & $1.61 \mathrm{E}-08$ & Staurosporine \\
\hline CDC7/DBF4 & 99.97 & 98.89 & $1.65 \mathrm{E}-08$ & Staurosporine \\
\hline CDK1/cyclin A & 94.97 & 94.80 & $3.26 \mathrm{E}-09$ & Staurosporine \\
\hline CDK1/cyclin B & 91.89 & 90.24 & $1.98 \mathrm{E}-09$ & Staurosporine \\
\hline CDK1/cyclin E & 94.27 & 93.45 & $9.46 \mathrm{E}-09$ & Staurosporine \\
\hline CDK14/cyclin Y (PFTK1) & 101.85 & 98.75 & $1.34 \mathrm{E}-07$ & Staurosporine \\
\hline CDK16/cyclin Y (PCTAIRE) & 102.72 & 100.37 & $2.12 \mathrm{E}-08$ & Staurosporine \\
\hline CDK17/cyclin Y (PCTK2) & 102.34 & 101.27 & $1.22 \mathrm{E}-08$ & Staurosporine \\
\hline CDK18/cyclin Y (PCTK3) & 96.98 & 95.09 & 3.37E-08 & Staurosporine \\
\hline CDK19/cyclin C & 102.65 & 102.00 & $1.74 \mathrm{E}-10$ & Staurosporine \\
\hline CDK2/cyclin A & 85.55 & 84.81 & $4.35 \mathrm{E}-10$ & Staurosporine \\
\hline CDK2/Cyclin A1 & 93.18 & 90.74 & $1.48 \mathrm{E}-09$ & Staurosporine \\
\hline CDK2/cyclin E & 105.53 & 102.00 & $3.48 \mathrm{E}-09$ & Staurosporine \\
\hline CDK2/cyclin E2 & 98.74 & 86.64 & $2.88 \mathrm{E}-09$ & Staurosporine \\
\hline CDK2/cyclin $\mathrm{O}$ & 103.45 & 102.57 & $2.09 \mathrm{E}-09$ & Staurosporine \\
\hline CDK3/cyclin E & 96.71 & 94.85 & 2.97E-09 & Staurosporine \\
\hline CDK3/cyclin E2 & 88.01 & 86.80 & $2.03 \mathrm{E}-09$ & Staurosporine \\
\hline CDK4/cyclin D1 & 84.16 & 81.20 & $1.23 \mathrm{E}-08$ & Staurosporine \\
\hline CDK4/cyclin D3 & 99.12 & 98.75 & $3.96 \mathrm{E}-08$ & Staurosporine \\
\hline $\mathrm{CDK} 5 / \mathrm{p} 25$ & 97.59 & 96.89 & $2.53 \mathrm{E}-09$ & Staurosporine \\
\hline CDK5/p35 & 93.57 & 93.06 & $2.04 \mathrm{E}-09$ & Staurosporine \\
\hline CDK6/cyclin D1 & 95.10 & 94.78 & 4.98E-09 & Staurosporine \\
\hline CDK6/cyclin D3 & 86.35 & 86.12 & $1.78 \mathrm{E}-08$ & Staurosporine \\
\hline CDK7/cyclin H & 101.50 & 101.48 & 4.94E-08 & Staurosporine \\
\hline CDK9/cyclin K & 100.60 & 98.40 & $1.62 \mathrm{E}-08$ & Staurosporine \\
\hline CDK9/cyclin T1 & 97.87 & 94.52 & $1.22 \mathrm{E}-08$ & Staurosporine \\
\hline CDK9/cyclin T2 & 103.07 & 102.80 & 4.73E-09 & Staurosporine \\
\hline CHK1 & 101.54 & 101.16 & $1.48 \mathrm{E}-10$ & Staurosporine \\
\hline CHK2 & 107.41 & 104.65 & $5.81 \mathrm{E}-09$ & Staurosporine \\
\hline CK1a1 & 100.56 & 99.83 & $4.55 \mathrm{E}-06$ & Staurosporine \\
\hline CK1a1L & 92.09 & 90.97 & $1.53 \mathrm{E}-06$ & Staurosporine \\
\hline CK1d & 95.42 & 94.22 & $3.25 \mathrm{E}-07$ & D4476 \\
\hline CK1epsilon & 89.13 & 88.01 & $3.09 \mathrm{E}-07$ & D4476 \\
\hline CK1g1 & 106.50 & 101.50 & $5.25 \mathrm{E}-06$ & Staurosporine \\
\hline
\end{tabular}




\begin{tabular}{|c|c|c|c|c|}
\hline CK1g2 & 100.48 & 99.95 & $3.70 \mathrm{E}-06$ & Staurosporine \\
\hline CK1g3 & 95.40 & 93.46 & $2.43 \mathrm{E}-06$ & Staurosporine \\
\hline $\mathrm{CK} 2 \mathrm{a}$ & 102.43 & 101.31 & $2.61 \mathrm{E}-07$ & GW5074 \\
\hline CK2a2 & 75.56 & 74.65 & $1.58 \mathrm{E}-07$ & Staurosporine \\
\hline CLK1 & 104.54 & 103.30 & $9.10 \mathrm{E}-09$ & Staurosporine \\
\hline CLK2 & 114.35 & 113.96 & $6.58 \mathrm{E}-09$ & Staurosporine \\
\hline CLK3 & 108.67 & 100.74 & $1.42 \mathrm{E}-06$ & Staurosporine \\
\hline CLK4 & 94.95 & 92.69 & $1.51 \mathrm{E}-08$ & Staurosporine \\
\hline СОТ1/MAP3К8 & 95.78 & 94.44 & $1.43 \mathrm{E}-05$ & Ro-31-8220 \\
\hline CSK & 102.38 & 100.32 & $1.33 \mathrm{E}-08$ & Staurosporine \\
\hline CTK/MATK & 76.70 & 76.65 & $1.20 \mathrm{E}-06$ & Staurosporine \\
\hline DAPK1 & 96.83 & 96.62 & $2.90 \mathrm{E}-08$ & Staurosporine \\
\hline DAPK2 & 95.05 & 94.58 & $3.01 \mathrm{E}-08$ & Staurosporine \\
\hline DCAMKL1 & 100.48 & 99.40 & $1.93 \mathrm{E}-07$ & Staurosporine \\
\hline DCAMKL2 & 87.89 & 86.73 & $4.68 \mathrm{E}-08$ & Staurosporine \\
\hline DDR1 & 82.06 & 80.79 & $2.06 \mathrm{E}-09$ & Staurosporine \\
\hline DDR2 & 111.45 & 107.70 & $1.92 \mathrm{E}-10$ & Staurosporine \\
\hline DLK/MAP3K12 & 86.90 & 85.92 & $5.60 \mathrm{E}-08$ & Staurosporine \\
\hline DMPK & 100.54 & 98.86 & $1.92 \mathrm{E}-08$ & Staurosporine \\
\hline DMPK2 & 104.47 & 104.24 & $2.72 \mathrm{E}-10$ & Staurosporine \\
\hline DRAK1/STK17A & 102.51 & 99.81 & $5.24 \mathrm{E}-08$ & Staurosporine \\
\hline DYRK1/DYRK1A & 99.18 & 96.46 & 3.09E-09 & Staurosporine \\
\hline DYRK1B & 99.17 & 93.21 & $8.82 \mathrm{E}-10$ & Staurosporine \\
\hline DYRK2 & 94.26 & 91.65 & $1.29 \mathrm{E}-07$ & Staurosporine \\
\hline DYRK3 & 103.12 & 102.51 & $2.90 \mathrm{E}-08$ & Staurosporine \\
\hline DYRK4 & 105.04 & 104.55 & $3.63 \mathrm{E}-06$ & GW5074 \\
\hline EGFR & 103.31 & 98.59 & $2.19 \mathrm{E}-07$ & Staurosporine \\
\hline EPHA1 & 93.51 & 92.31 & $1.74 \mathrm{E}-07$ & Staurosporine \\
\hline EPHA2 & 97.37 & 95.94 & $5.17 \mathrm{E}-08$ & Staurosporine \\
\hline EPHA3 & 103.40 & 102.11 & 4.81E-08 & Staurosporine \\
\hline EPHA4 & 102.15 & 101.99 & $2.03 \mathrm{E}-08$ & Staurosporine \\
\hline EPHA5 & 98.55 & 98.32 & $2.15 \mathrm{E}-08$ & Staurosporine \\
\hline ЕРНА6 & 95.01 & 94.95 & $5.47 \mathrm{E}-08$ & Staurosporine \\
\hline EPHA7 & 90.00 & 88.83 & $3.16 \mathrm{E}-08$ & Staurosporine \\
\hline EPHA8 & 103.09 & 103.02 & $2.42 \mathrm{E}-07$ & Staurosporine \\
\hline EPHB1 & 96.94 & 96.51 & $3.87 \mathrm{E}-08$ & Staurosporine \\
\hline EPHB2 & 97.69 & 96.79 & $8.22 \mathrm{E}-08$ & Staurosporine \\
\hline EPHB3 & 94.38 & 94.13 & $1.08 \mathrm{E}-06$ & Staurosporine \\
\hline EPHB4 & 84.94 & 82.98 & $1.91 \mathrm{E}-07$ & Staurosporine \\
\hline ERBB2/HER2 & 92.93 & 92.24 & $2.51 \mathrm{E}-07$ & Staurosporine \\
\hline ERBB4/HER4 & 89.14 & 88.66 & $2.63 \mathrm{E}-07$ & Staurosporine \\
\hline ERK1 & 92.88 & 92.81 & $1.74 \mathrm{E}-08$ & SCH772984 \\
\hline
\end{tabular}




\begin{tabular}{|c|c|c|c|c|}
\hline ERK2/MAPK1 & 99.07 & 98.80 & 1.39E-08 & SCH772984 \\
\hline ERK5/MAPK7 & 99.70 & 97.06 & $2.49 \mathrm{E}-05$ & Staurosporine \\
\hline ERK7/MAPK15 & 115.74 & 113.18 & $3.20 \mathrm{E}-09$ & Staurosporine \\
\hline ERN1/IRE1 & 95.58 & 95.27 & $9.65 \mathrm{E}-08$ & Staurosporine \\
\hline ERN2/IRE2 & 107.74 & 103.44 & $1.91 \mathrm{E}-08$ & Staurosporine \\
\hline FAK/PTK2 & 99.73 & 97.49 & $1.52 \mathrm{E}-08$ & Staurosporine \\
\hline FER & 99.32 & 98.85 & $1.03 \mathrm{E}-09$ & Staurosporine \\
\hline FES/FPS & 99.02 & 98.91 & $2.50 \mathrm{E}-09$ & Staurosporine \\
\hline FGFR1 & 105.90 & 105.89 & $1.01 \mathrm{E}-08$ & Staurosporine \\
\hline FGFR2 & 93.22 & 93.21 & 3.91E-09 & Staurosporine \\
\hline FGFR3 & 99.84 & 98.62 & $2.06 \mathrm{E}-08$ & Staurosporine \\
\hline FGFR4 & 117.54 & 114.05 & $1.11 \mathrm{E}-07$ & Staurosporine \\
\hline FGR & 98.02 & 95.89 & $9.79 \mathrm{E}-10$ & Staurosporine \\
\hline FLT1/VEGFR1 & 79.74 & 79.50 & 8.33E-09 & Staurosporine \\
\hline FLT3 & 86.20 & 85.29 & $1.16 \mathrm{E}-09$ & Staurosporine \\
\hline FLT4/VEGFR3 & 101.08 & 100.53 & $2.99 \mathrm{E}-09$ & Staurosporine \\
\hline FMS & 88.28 & 86.36 & $2.06 \mathrm{E}-09$ & Staurosporine \\
\hline FRK/PTK5 & 99.52 & 98.63 & $1.39 \mathrm{E}-08$ & Staurosporine \\
\hline FYN & 91.84 & 91.67 & 1.69E-09 & Staurosporine \\
\hline GCK/MAP4K2 & 104.12 & 103.49 & $6.58 \mathrm{E}-10$ & Staurosporine \\
\hline GLK/MAP4K3 & 97.04 & 96.28 & $6.50 \mathrm{E}-11$ & Staurosporine \\
\hline GRK1 & 103.44 & 98.96 & 4.94E-08 & Staurosporine \\
\hline GRK2 & 103.59 & 103.38 & $1.01 \mathrm{E}-06$ & Staurosporine \\
\hline GRK3 & 106.94 & 106.49 & $1.03 \mathrm{E}-06$ & Staurosporine \\
\hline GRK4 & 106.55 & 105.62 & $1.38 \mathrm{E}-07$ & Staurosporine \\
\hline GRK5 & 109.97 & 107.04 & 4.57E-08 & Staurosporine \\
\hline GRK6 & 104.67 & 104.46 & $4.14 \mathrm{E}-08$ & Staurosporine \\
\hline GRK7 & 109.42 & 109.22 & 7.72E-09 & Staurosporine \\
\hline GSK3a & 100.95 & 100.79 & 8.57E-09 & Staurosporine \\
\hline GSK3b & 102.25 & 100.00 & $1.76 \mathrm{E}-08$ & Staurosporine \\
\hline Haspin & 104.27 & 100.92 & $3.41 \mathrm{E}-08$ & Staurosporine \\
\hline HCK & 93.91 & 93.33 & $1.56 \mathrm{E}-09$ & Staurosporine \\
\hline HGK/MAP4K4 & 105.38 & 102.02 & $4.43 \mathrm{E}-10$ & Staurosporine \\
\hline HIPK1 & 114.88 & 113.77 & $1.96 \mathrm{E}-06$ & Ro-31-8220 \\
\hline HIPK2 & 110.65 & 106.39 & $1.97 \mathrm{E}-06$ & Staurosporine \\
\hline HIPK3 & 110.09 & 108.38 & $7.25 \mathrm{E}-07$ & Staurosporine \\
\hline HIPK4 & 108.11 & 106.81 & 4.43E-07 & Staurosporine \\
\hline HPK1/MAP4K1 & 103.98 & 102.32 & $1.16 \mathrm{E}-07$ & Ro-31-8220 \\
\hline IGF1R & 92.54 & 91.68 & $5.49 \mathrm{E}-08$ & Staurosporine \\
\hline IKKa/CHUK & 102.20 & 100.70 & $3.46 \mathrm{E}-07$ & Staurosporine \\
\hline IKKb/IKBKB & 98.91 & 94.16 & $5.73 \mathrm{E}-07$ & Staurosporine \\
\hline IKKe/IKBKE & 95.25 & 95.24 & 4.19E-10 & Staurosporine \\
\hline
\end{tabular}




\begin{tabular}{|c|c|c|c|c|}
\hline IR & 103.90 & 102.29 & 2.63E-08 & Staurosporine \\
\hline IRAK1 & 107.89 & 107.17 & $3.08 \mathrm{E}-08$ & Staurosporine \\
\hline IRAK4 & 98.17 & 93.16 & 8.82E-09 & Staurosporine \\
\hline IRR/INSRR & 84.01 & 81.13 & $9.25 \mathrm{E}-09$ & Staurosporine \\
\hline ITK & 104.67 & 104.41 & $1.11 \mathrm{E}-08$ & Staurosporine \\
\hline JAK1 & 98.36 & 97.75 & $5.51 \mathrm{E}-10$ & Staurosporine \\
\hline JAK2 & 99.04 & 97.30 & $1.64 \mathrm{E}-10$ & Staurosporine \\
\hline JAK3 & 105.61 & 103.13 & $5.84 \mathrm{E}-11$ & Staurosporine \\
\hline JNK1 & 99.48 & 99.17 & $3.34 \mathrm{E}-06$ & Staurosporine \\
\hline JNK2 & 105.16 & 103.55 & $1.35 \mathrm{E}-06$ & Staurosporine \\
\hline JNK3 & 96.33 & 94.40 & $2.57 \mathrm{E}-07$ & JNKi VIII \\
\hline KDR/VEGFR2 & 103.87 & 103.45 & $1.38 \mathrm{E}-08$ & Staurosporine \\
\hline KHS/MAP4K5 & 103.58 & 100.46 & $4.21 \mathrm{E}-10$ & Staurosporine \\
\hline KSR1 & 87.52 & 85.46 & $9.80 \mathrm{E}-06$ & Staurosporine \\
\hline KSR2 & 95.42 & 94.78 & $6.68 \mathrm{E}-06$ & Staurosporine \\
\hline LATS1 & 99.38 & 98.61 & $1.48 \mathrm{E}-08$ & Staurosporine \\
\hline LATS2 & 101.55 & 98.86 & 2.07E-09 & Staurosporine \\
\hline LCK & 96.03 & 95.74 & $3.72 \mathrm{E}-09$ & Staurosporine \\
\hline LCK2/ICK & 101.98 & 100.45 & $1.08 \mathrm{E}-07$ & Staurosporine \\
\hline LIMK1 & 90.36 & 89.77 & $7.16 \mathrm{E}-10$ & Staurosporine \\
\hline LIMK2 & 94.80 & 94.51 & $6.48 \mathrm{E}-08$ & Staurosporine \\
\hline LKB1 & 83.65 & 82.72 & $1.12 \mathrm{E}-07$ & Staurosporine \\
\hline LOK/STK10 & 100.43 & 100.25 & $9.90 \mathrm{E}-08$ & Ro-31-8220 \\
\hline LRRK2 & 100.22 & 97.68 & 4.85E-09 & Staurosporine \\
\hline LYN & 93.70 & 92.60 & $9.82 \mathrm{E}-10$ & Staurosporine \\
\hline LYN B & 96.32 & 91.42 & $5.69 \mathrm{E}-09$ & Staurosporine \\
\hline MAK & 96.01 & 95.60 & $2.19 \mathrm{E}-08$ & Staurosporine \\
\hline MAPKAPK2 & 101.09 & 99.95 & $1.50 \mathrm{E}-07$ & Staurosporine \\
\hline MAPKAPK3 & 101.47 & 100.92 & $1.80 \mathrm{E}-06$ & Staurosporine \\
\hline MAPKAPK5/PRAK & 97.85 & 95.78 & $2.81 \mathrm{E}-07$ & Staurosporine \\
\hline MARK1 & 105.58 & 104.65 & $1.19 \mathrm{E}-10$ & Staurosporine \\
\hline MARK2/PAR-1Ba & 93.49 & 91.91 & $6.46 \mathrm{E}-11$ & Staurosporine \\
\hline MARK3 & 106.69 & 105.68 & $4.30 \mathrm{E}-10$ & Staurosporine \\
\hline MARK4 & 110.52 & 108.97 & $8.03 \mathrm{E}-11$ & Staurosporine \\
\hline MEK1 & 98.80 & 98.34 & $3.90 \mathrm{E}-08$ & Staurosporine \\
\hline MEK2 & 100.00 & 96.68 & $3.66 \mathrm{E}-08$ & Staurosporine \\
\hline MEK3 & 107.11 & 102.87 & $1.03 \mathrm{E}-08$ & Staurosporine \\
\hline MEK5 & 102.83 & 100.12 & $3.24 \mathrm{E}-08$ & Staurosporine \\
\hline MEKK1 & 112.94 & 107.40 & 7.03E-07 & Staurosporine \\
\hline MEKK2 & 99.48 & 98.79 & $3.80 \mathrm{E}-08$ & Staurosporine \\
\hline MEKK3 & 93.79 & 91.13 & 7.19E-08 & Staurosporine \\
\hline MEKK6 & 110.07 & 105.17 & $3.25 \mathrm{E}-07$ & Staurosporine \\
\hline
\end{tabular}




\begin{tabular}{|c|c|c|c|c|}
\hline MELK & 98.56 & 94.83 & $8.14 \mathrm{E}-10$ & Staurosporine \\
\hline MINK/MINK1 & 92.25 & 91.61 & $6.97 \mathrm{E}-10$ & Staurosporine \\
\hline MKK4 & 109.10 & 108.12 & $1.56 \mathrm{E}-06$ & Staurosporine \\
\hline MKK6 & 100.63 & 97.49 & $8.81 \mathrm{E}-09$ & Staurosporine \\
\hline MKK7 & 101.40 & 100.79 & $1.59 \mathrm{E}-06$ & Staurosporine \\
\hline MLCK/MYLK & 93.65 & 93.45 & $5.63 \mathrm{E}-08$ & Staurosporine \\
\hline MLCK2/MYLK2 & 98.10 & 94.36 & $1.62 \mathrm{E}-08$ & Staurosporine \\
\hline MLK1/MAP3K9 & 93.99 & 92.47 & $9.14 \mathrm{E}-10$ & Staurosporine \\
\hline MLK2/MAP3K10 & 87.67 & 87.52 & $2.59 \mathrm{E}-09$ & Staurosporine \\
\hline MLK3/MAP3K11 & 94.95 & 94.22 & $1.82 \mathrm{E}-09$ & Staurosporine \\
\hline MLK4 & 100.42 & 99.41 & $3.82 \mathrm{E}-06$ & Staurosporine \\
\hline MNK1 & 106.13 & 102.92 & $5.05 \mathrm{E}-08$ & Staurosporine \\
\hline MNK2 & 120.59 & 112.29 & $1.79 \mathrm{E}-08$ & Staurosporine \\
\hline MRCKa/CDC42BPA & 103.67 & 101.56 & 3.59E-09 & Staurosporine \\
\hline MRCKb/CDC42BPB & 92.13 & 87.79 & $2.62 \mathrm{E}-09$ & Staurosporine \\
\hline MSK1/RPS6KA5 & 98.37 & 98.19 & $5.09 \mathrm{E}-10$ & Staurosporine \\
\hline MSK2/RPS6KA4 & 99.39 & 97.15 & $3.10 \mathrm{E}-09$ & Staurosporine \\
\hline MSSK1/STK23 & 99.29 & 98.07 & $1.97 \mathrm{E}-06$ & Staurosporine \\
\hline MST1/STK4 & 104.01 & 103.13 & 4.39E-10 & Staurosporine \\
\hline MST2/STK3 & 92.97 & 91.92 & 5.34E-09 & Staurosporine \\
\hline MST3/STK24 & 121.28 & 120.62 & 4.12E-09 & Staurosporine \\
\hline MST4 & 78.34 & 76.55 & 7.04E-09 & Staurosporine \\
\hline MUSK & 112.54 & 110.13 & $3.15 \mathrm{E}-07$ & Staurosporine \\
\hline MYLK3 & 105.52 & 104.95 & $1.92 \mathrm{E}-07$ & Staurosporine \\
\hline MYLK4 & 101.97 & 100.26 & $8.42 \mathrm{E}-08$ & Staurosporine \\
\hline MYO3A & 99.75 & 99.11 & 3.57E-08 & Staurosporine \\
\hline MYO3b & 106.83 & 106.78 & $6.10 \mathrm{E}-09$ & Staurosporine \\
\hline NEK1 & 82.42 & 78.68 & 1.19E-08 & Staurosporine \\
\hline NEK11 & 85.02 & 84.20 & $2.54 \mathrm{E}-07$ & Staurosporine \\
\hline NEK2 & 106.85 & 104.72 & 4.81E-07 & Staurosporine \\
\hline NEK3 & 104.02 & 103.07 & $6.55 \mathrm{E}-05$ & Staurosporine \\
\hline NEK4 & 98.34 & 97.18 & $1.19 \mathrm{E}-07$ & Staurosporine \\
\hline NEK5 & 132.72 & 132.44 & $4.52 \mathrm{E}-08$ & Staurosporine \\
\hline NEK6 & 92.85 & 92.45 & $1.89 \mathrm{E}-05$ & PKR Inhibitor \\
\hline NEK7 & 94.36 & 91.24 & $1.93 \mathrm{E}-05$ & PKR Inhibitor \\
\hline NEK8 & 90.43 & 88.36 & $1.24 \mathrm{E}-08$ & Staurosporine \\
\hline NEK9 & 96.08 & 96.03 & $1.50 \mathrm{E}-07$ & Staurosporine \\
\hline NIM1 & 99.38 & 98.32 & $1.28 \mathrm{E}-07$ & Staurosporine \\
\hline NLK & 102.17 & 98.59 & 7.61E-08 & Staurosporine \\
\hline OSR1/OXSR1 & 99.25 & 94.29 & $9.93 \mathrm{E}-08$ & Staurosporine \\
\hline P38a/MAPK14 & 105.07 & 101.11 & $1.85 \mathrm{E}-08$ & SB202190 \\
\hline P38b/MAPK11 & 100.87 & 99.35 & $6.14 \mathrm{E}-08$ & SB202190 \\
\hline
\end{tabular}




\begin{tabular}{|c|c|c|c|c|}
\hline P38d/MAPK13 & 129.76 & 125.43 & $2.20 \mathrm{E}-07$ & Staurosporine \\
\hline P38g & 103.61 & 101.31 & $1.21 \mathrm{E}-07$ & Staurosporine \\
\hline p70S6K/RPS6KB1 & 99.47 & 99.45 & 4.61E-10 & Staurosporine \\
\hline p70S6Kb/RPS6KB2 & 103.68 & 103.19 & $3.15 \mathrm{E}-09$ & Staurosporine \\
\hline PAK1 & 90.91 & 87.98 & $3.27 \mathrm{E}-10$ & Staurosporine \\
\hline PAK2 & 98.92 & 97.68 & $1.45 \mathrm{E}-09$ & Staurosporine \\
\hline PAK3 & 101.24 & 99.19 & $6.70 \mathrm{E}-10$ & Staurosporine \\
\hline PAK4 & 111.06 & 110.22 & $2.34 \mathrm{E}-08$ & Staurosporine \\
\hline PAK5 & 86.77 & 85.93 & 5.04E-09 & Staurosporine \\
\hline PAK6 & 76.49 & 75.18 & 5.93E-08 & Staurosporine \\
\hline PASK & 94.39 & 93.58 & $1.59 \mathrm{E}-08$ & Staurosporine \\
\hline PBK/TOPK & 98.75 & 97.81 & 8.94E-08 & Staurosporine \\
\hline PDGFRa & 104.48 & 102.46 & 7.84E-10 & Staurosporine \\
\hline PDGFRb & 91.83 & 89.16 & 2.77E-09 & Staurosporine \\
\hline PDK1/PDPK1 & 100.84 & 99.95 & $7.60 \mathrm{E}-10$ & Staurosporine \\
\hline PEAK1 & 94.04 & 93.24 & $3.28 \mathrm{E}-09$ & Staurosporine \\
\hline PHKg1 & 81.72 & 80.93 & 2.07E-09 & Staurosporine \\
\hline PHKg2 & 87.51 & 82.02 & $4.91 \mathrm{E}-10$ & Staurosporine \\
\hline PIM1 & 91.01 & 90.75 & $3.44 \mathrm{E}-09$ & Staurosporine \\
\hline PIM2 & 92.42 & 91.37 & $3.66 \mathrm{E}-08$ & Staurosporine \\
\hline PIM3 & 92.99 & 92.31 & 8.97E-11 & Staurosporine \\
\hline PKA & 90.94 & 88.66 & $1.23 \mathrm{E}-09$ & Staurosporine \\
\hline PKAcb & 96.53 & 95.14 & $8.23 \mathrm{E}-10$ & Staurosporine \\
\hline PKAcg & 95.78 & 93.29 & $2.81 \mathrm{E}-09$ & Staurosporine \\
\hline $\mathrm{PKCa}$ & 92.66 & 91.11 & $4.25 \mathrm{E}-10$ & Staurosporine \\
\hline PKCb1 & 97.38 & 97.08 & 3.72E-09 & Staurosporine \\
\hline $\mathrm{PKCb} 2$ & 97.69 & 94.38 & $1.74 \mathrm{E}-09$ & Staurosporine \\
\hline PKCd & 94.79 & 93.37 & $1.21 \mathrm{E}-10$ & Staurosporine \\
\hline PKCepsilon & 96.12 & 93.83 & $2.02 \mathrm{E}-10$ & Staurosporine \\
\hline PKCeta & 99.18 & 98.09 & $6.84 \mathrm{E}-10$ & Staurosporine \\
\hline $\mathrm{PKCg}$ & 101.46 & 98.73 & 1.63E-09 & Staurosporine \\
\hline PKCiota & 112.76 & 111.56 & $2.43 \mathrm{E}-08$ & Staurosporine \\
\hline PKCmu/PRKD1 & 107.76 & 107.13 & $1.43 \mathrm{E}-09$ & Staurosporine \\
\hline PKCnu/PRKD3 & 92.50 & 91.76 & $1.14 \mathrm{E}-09$ & Staurosporine \\
\hline PKCtheta & 108.45 & 106.63 & $2.34 \mathrm{E}-09$ & Staurosporine \\
\hline PKCzeta & 100.21 & 98.97 & $6.26 \mathrm{E}-08$ & Staurosporine \\
\hline PKD2/PRKD2 & 95.54 & 94.90 & 1.13E-09 & Staurosporine \\
\hline PKG1a & 83.02 & 81.45 & $1.06 \mathrm{E}-09$ & Staurosporine \\
\hline PKG1b & 99.30 & 99.21 & $2.88 \mathrm{E}-09$ & Staurosporine \\
\hline PKG2/PRKG2 & 109.21 & 102.41 & 1.18E-09 & Staurosporine \\
\hline PKN1/PRK1 & 102.40 & 101.46 & $1.88 \mathrm{E}-10$ & Staurosporine \\
\hline PKN2/PRK2 & 104.07 & 103.59 & $3.14 \mathrm{E}-09$ & Staurosporine \\
\hline
\end{tabular}




\begin{tabular}{|c|c|c|c|c|}
\hline PKN3/PRK3 & 102.92 & 101.43 & $9.78 \mathrm{E}-09$ & Staurosporine \\
\hline PLK1 & 95.98 & 93.84 & $2.75 \mathrm{E}-07$ & Staurosporine \\
\hline PLK2 & 100.47 & 100.26 & $3.73 \mathrm{E}-07$ & Staurosporine \\
\hline PLK3 & 106.44 & 105.68 & $2.88 \mathrm{E}-08$ & BI2536 \\
\hline PLK4/SAK & 98.60 & 97.31 & $8.41 \mathrm{E}-09$ & Staurosporine \\
\hline PRKX & 100.54 & 98.25 & 2.03E-09 & Staurosporine \\
\hline PYK2 & 82.11 & 80.19 & $6.14 \mathrm{E}-09$ & Staurosporine \\
\hline RAF1 & 96.78 & 96.26 & $9.88 \mathrm{E}-09$ & GW5074 \\
\hline RET & 114.49 & 110.67 & 2.70E-09 & Staurosporine \\
\hline RIPK2 & 99.78 & 96.02 & $1.42 \mathrm{E}-07$ & Staurosporine \\
\hline RIPK3 & 103.39 & 101.36 & $2.96 \mathrm{E}-06$ & GW5074 \\
\hline RIPK4 & 101.73 & 98.10 & 8.57E-07 & Staurosporine \\
\hline RIPK5 & 102.00 & 100.78 & $1.00 \mathrm{E}-07$ & Staurosporine \\
\hline ROCK1 & 95.70 & 94.87 & 7.27E-10 & Staurosporine \\
\hline ROCK2 & 99.92 & 99.49 & $4.08 \mathrm{E}-10$ & Staurosporine \\
\hline RON/MST1R & 108.88 & 108.10 & $3.55 \mathrm{E}-07$ & Staurosporine \\
\hline ROS/ROS1 & 102.10 & 101.05 & $4.51 \mathrm{E}-10$ & Staurosporine \\
\hline RSK1 & 94.83 & 91.66 & $8.24 \mathrm{E}-11$ & Staurosporine \\
\hline RSK2 & 95.09 & 92.95 & $3.85 \mathrm{E}-10$ & Staurosporine \\
\hline RSK3 & 103.88 & 101.78 & $4.10 \mathrm{E}-10$ & Staurosporine \\
\hline RSK4 & 96.93 & 96.79 & $1.30 \mathrm{E}-10$ & Staurosporine \\
\hline SBK1 & 108.87 & 107.90 & $9.83 \mathrm{E}-08$ & Staurosporine \\
\hline SGK1 & 91.38 & 87.49 & 8.59E-09 & Staurosporine \\
\hline SGK2 & 90.43 & 89.23 & $1.36 \mathrm{E}-08$ & Staurosporine \\
\hline SGK3/SGKL & 91.79 & 86.69 & $5.82 \mathrm{E}-08$ & Staurosporine \\
\hline SIK1 & 98.80 & 93.63 & $6.69 \mathrm{E}-10$ & Staurosporine \\
\hline SIK2 & 114.70 & 105.38 & $1.17 \mathrm{E}-10$ & Staurosporine \\
\hline SIK3 & 96.46 & 95.08 & $6.44 \mathrm{E}-10$ & Staurosporine \\
\hline SLK/STK2 & 93.68 & 93.05 & $1.88 \mathrm{E}-08$ & Staurosporine \\
\hline SNARK/NUAK2 & 89.50 & 87.14 & $1.72 \mathrm{E}-09$ & Staurosporine \\
\hline SNRK & 106.77 & 106.68 & $1.48 \mathrm{E}-08$ & Staurosporine \\
\hline SRMS & 85.97 & 85.18 & $6.84 \mathrm{E}-06$ & Staurosporine \\
\hline SRPK1 & 112.72 & 111.59 & $1.29 \mathrm{E}-07$ & Staurosporine \\
\hline SRPK2 & 101.93 & 101.51 & $3.41 \mathrm{E}-07$ & Staurosporine \\
\hline SSTK/TSSK6 & 94.45 & 92.35 & $2.02 \mathrm{E}-07$ & Staurosporine \\
\hline STK16 & 121.67 & 117.38 & $2.97 \mathrm{E}-07$ & Staurosporine \\
\hline STK21/CIT & 107.64 & 106.36 & $5.20 \mathrm{E}-07$ & Staurosporine \\
\hline STK22D/TSSK1 & 97.65 & 96.68 & $5.65 \mathrm{E}-11$ & Staurosporine \\
\hline STK25/YSK1 & 97.03 & 96.09 & $3.88 \mathrm{E}-09$ & Staurosporine \\
\hline STK32B/YANK2 & 106.94 & 104.97 & $3.90 \mathrm{E}-08$ & Staurosporine \\
\hline STK32C/YANK3 & 114.48 & 110.53 & $3.68 \mathrm{E}-07$ & Staurosporine \\
\hline STK33 & 92.45 & 91.34 & $2.76 \mathrm{E}-08$ & Staurosporine \\
\hline
\end{tabular}




\begin{tabular}{|c|c|c|c|c|}
\hline STK38/NDR1 & 103.87 & 103.76 & $7.22 \mathrm{E}-10$ & Staurosporine \\
\hline STK38L/NDR2 & 96.93 & 96.04 & $9.62 \mathrm{E}-10$ & Staurosporine \\
\hline STK39/STLK3 & 101.44 & 100.68 & $2.74 \mathrm{E}-08$ & Staurosporine \\
\hline SYK & 96.95 & 96.60 & $2.27 \mathrm{E}-10$ & Staurosporine \\
\hline TAK1 & 101.01 & 99.59 & $6.05 \mathrm{E}-08$ & Staurosporine \\
\hline TAOK1 & 118.78 & 117.48 & $1.12 \mathrm{E}-09$ & Staurosporine \\
\hline TAOK2/TAO1 & 114.05 & 110.95 & 4.27E-09 & Staurosporine \\
\hline TAOK3/JIK & 100.07 & 98.93 & $4.26 \mathrm{E}-10$ & Staurosporine \\
\hline TBK1 & 90.92 & 88.38 & $2.50 \mathrm{E}-09$ & Staurosporine \\
\hline TEC & 93.37 & 93.06 & $9.14 \mathrm{E}-08$ & Staurosporine \\
\hline TESK1 & 112.81 & 110.19 & 2.91E-07 & Staurosporine \\
\hline TESK2 & 105.34 & 104.08 & $1.96 \mathrm{E}-05$ & Staurosporine \\
\hline TGFBR2 & 117.80 & 116.45 & $1.83 \mathrm{E}-07$ & LDN193189 \\
\hline TIE2/TEK & 98.95 & 98.87 & 8.92E-08 & Staurosporine \\
\hline TLK1 & 120.48 & 114.10 & $3.22 \mathrm{E}-08$ & Staurosporine \\
\hline TLK2 & 97.46 & 96.59 & $2.60 \mathrm{E}-09$ & Staurosporine \\
\hline TNIK & 79.15 & 77.50 & $3.84 \mathrm{E}-10$ & Staurosporine \\
\hline TNK1 & 105.69 & 104.59 & 4.05E-09 & Staurosporine \\
\hline TRKA & 93.21 & 91.08 & $3.24 \mathrm{E}-09$ & Staurosporine \\
\hline TRKB & 106.78 & 102.68 & $8.87 \mathrm{E}-11$ & Staurosporine \\
\hline TRKC & 83.09 & 82.24 & $1.99 \mathrm{E}-10$ & Staurosporine \\
\hline TSSK2 & 106.23 & 104.88 & $3.50 \mathrm{E}-08$ & Staurosporine \\
\hline TSSK3/STK22C & 94.07 & 93.44 & $1.37 \mathrm{E}-08$ & Staurosporine \\
\hline TTBK1 & 101.17 & 100.81 & $1.53 \mathrm{E}-05$ & SB202190 \\
\hline TTBK2 & 112.03 & 111.60 & $3.60 \mathrm{E}-06$ & SB202190 \\
\hline TXK & 91.49 & 89.67 & $2.43 \mathrm{E}-08$ & Staurosporine \\
\hline TYK1/LTK & 90.52 & 88.08 & $1.25 \mathrm{E}-08$ & Staurosporine \\
\hline TYK2 & 88.01 & 86.63 & $1.42 \mathrm{E}-10$ & Staurosporine \\
\hline TYRO3/SKY & 96.71 & 95.48 & $5.69 \mathrm{E}-09$ & Staurosporine \\
\hline ULK1 & 107.82 & 107.39 & $9.08 \mathrm{E}-09$ & Staurosporine \\
\hline ULK2 & 106.09 & 103.89 & $1.34 \mathrm{E}-09$ & Staurosporine \\
\hline ULK3 & 99.74 & 99.36 & $3.78 \mathrm{E}-09$ & Staurosporine \\
\hline VRK1 & 90.15 & 86.26 & $5.42 \mathrm{E}-07$ & Ro-31-8220 \\
\hline VRK2 & 103.01 & 102.06 & $2.63 \mathrm{E}-05$ & Ro-31-8220 \\
\hline WEE1 & 99.34 & 98.37 & $3.36 \mathrm{E}-08$ & Wee-1 Inhibitor \\
\hline WNK1 & 90.93 & 89.61 & $2.06 \mathrm{E}-05$ & Staurosporine \\
\hline WNK2 & 114.82 & 114.73 & $1.90 \mathrm{E}-06$ & Staurosporine \\
\hline WNK3 & 100.64 & 100.34 & $1.44 \mathrm{E}-06$ & Wee-1 Inhibitor \\
\hline YES/YES1 & 97.99 & 97.84 & $3.74 \mathrm{E}-09$ & Staurosporine \\
\hline YSK4/MAP3K19 & 102.30 & 98.31 & $1.42 \mathrm{E}-08$ & Staurosporine \\
\hline ZAK/MLTK & 106.56 & 106.43 & $1.34 \mathrm{E}-06$ & GW5074 \\
\hline ZAP70 & 97.36 & 96.26 & $5.05 \mathrm{E}-09$ & Staurosporine \\
\hline
\end{tabular}




\begin{tabular}{|l|l|l|l|l|}
\hline ZIPK/DAPK3 & 105.03 & 103.83 & $5.89 \mathrm{E}-09$ & Staurosporine \\
\hline
\end{tabular}

\section{Cell-based assay of IDO1 inhibitors in HeLa cell}

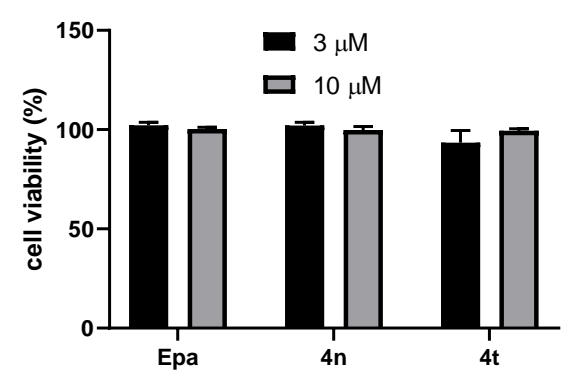

Figure S3. Cytotoxicity of representative compounds to HeLa cell

\section{Antitumor activity assay of candidate in vivo}

\section{(1) Antitumor results in CT26 mice xenograft model}

Table S3 TGI of Epa, 4n and 4t in a CT26 mice xenograft model (Day 28)

\begin{tabular}{cccc}
\hline Group & Tumor volumn $\left(\mathrm{mm}^{3}\right)$ & T/C $(\%)$ & TGI (\%) \\
\hline Ctrl & $1057 \pm 77$ & - & - \\
Epa $(50 \mathrm{mg} / \mathrm{kg})$ & $535 \pm 24$ & 50.6 & 49.4 \\
$\mathbf{4 n}(50 \mathrm{mg} / \mathrm{kg})$ & $356 \pm 9$ & 33.7 & 66.3 \\
$\mathbf{4 t}(50 \mathrm{mg} / \mathrm{kg})$ & $320 \pm 11$ & 30.3 & 69.7 \\
\hline
\end{tabular}

(2) Antitumor results of HCT116 nude mice xenograft model
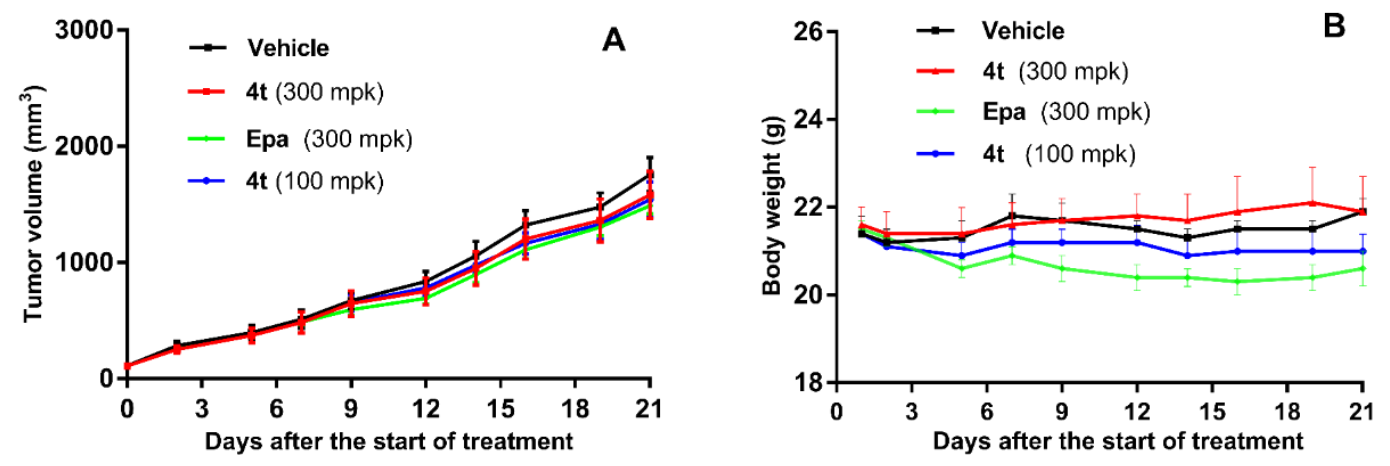

Figure S4. The effect of different compounds in HCT116 nude mice xenograft model. (A) The mouse body weight was measured every 3 days. compared with ctrl group. (B) The tumor volume for four groups of animals were compared. 
7. ${ }^{1} \mathrm{H}$ NMR, ${ }^{13} \mathrm{C}$ NMR Spectral of All Target Compounds

${ }^{1} \mathrm{H}$ NMR of $4 \mathrm{a}$
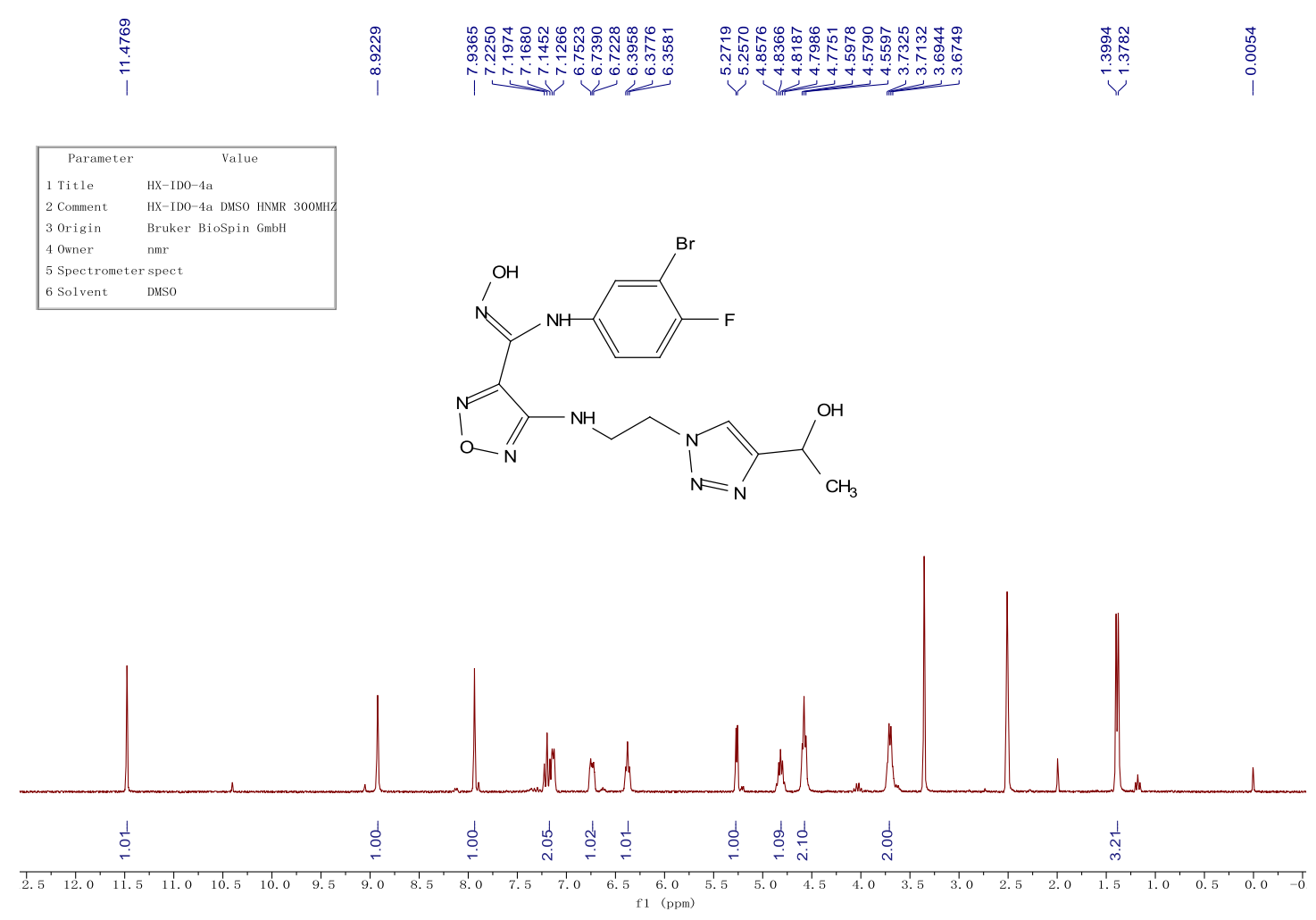

${ }^{13} \mathrm{C}$ NMR of $4 a$

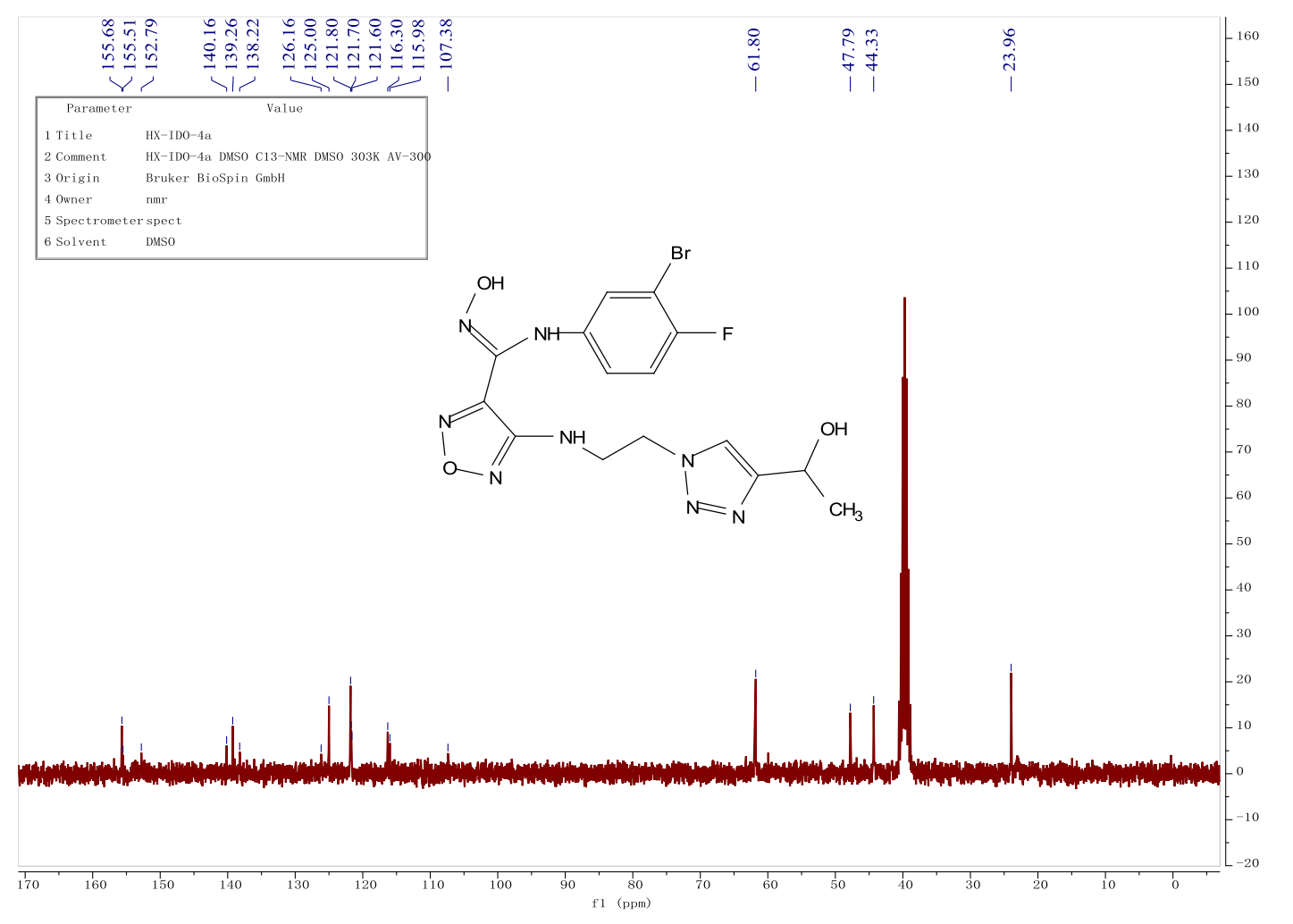


${ }^{1} \mathrm{H}$ NMR of $\mathbf{4 b}$

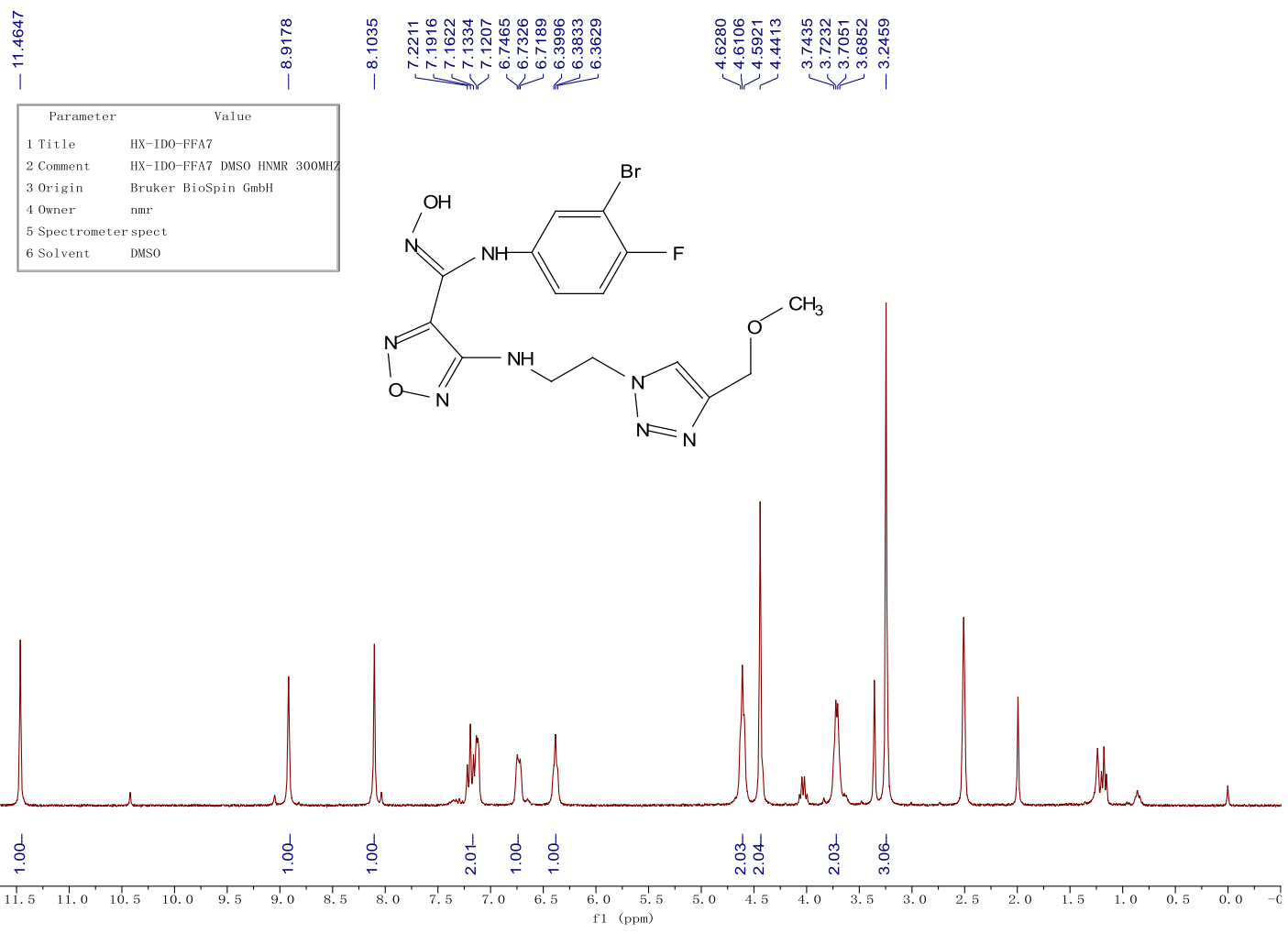

${ }^{13} \mathrm{C}$ NMR of $\mathbf{4 b}$

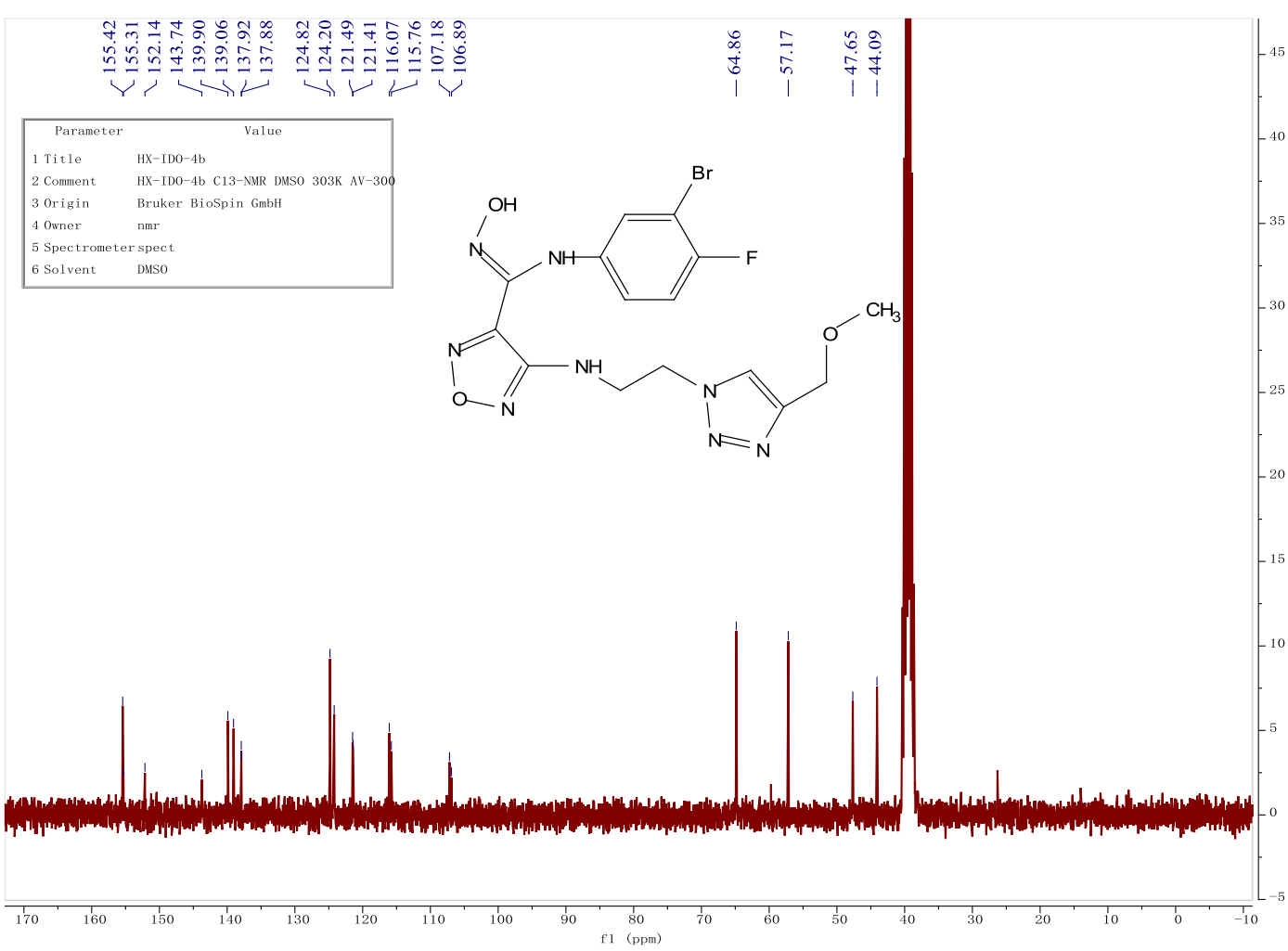


${ }^{1} \mathrm{H}$ NMR of $4 c$

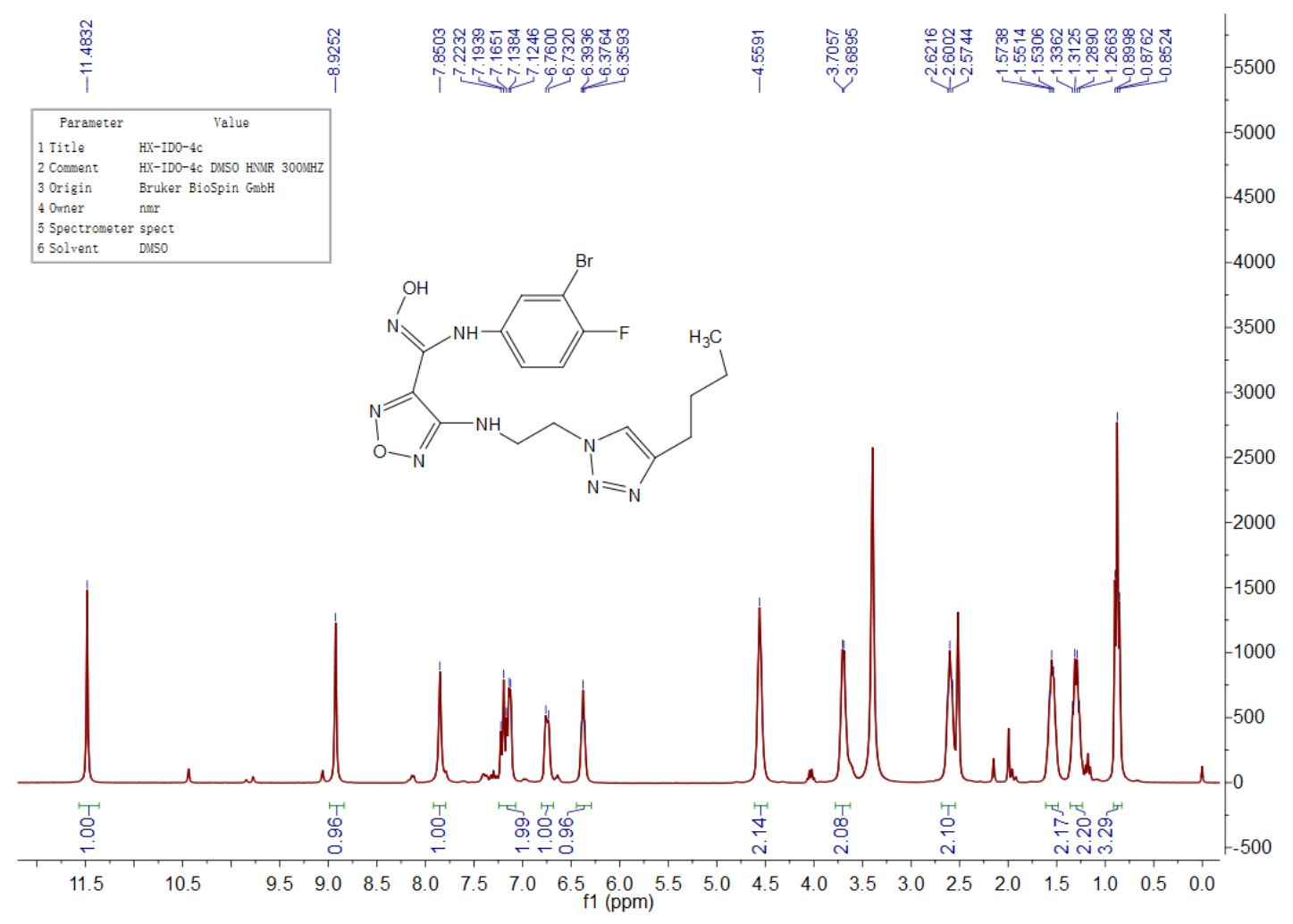

${ }^{13} \mathrm{C}$ NMR of $4 c$
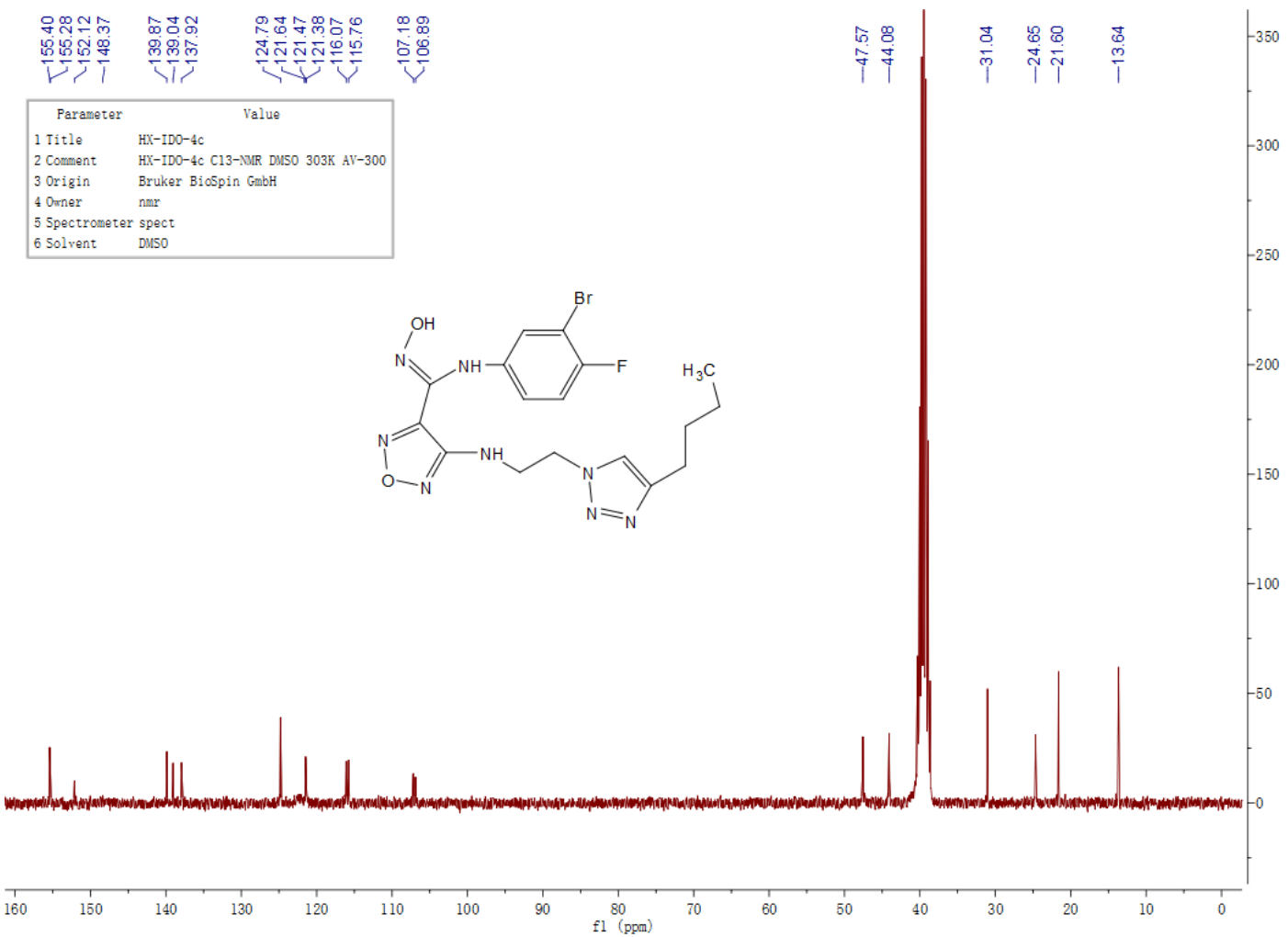
${ }^{1} \mathrm{H}$ NMR of $\mathbf{4 d}$

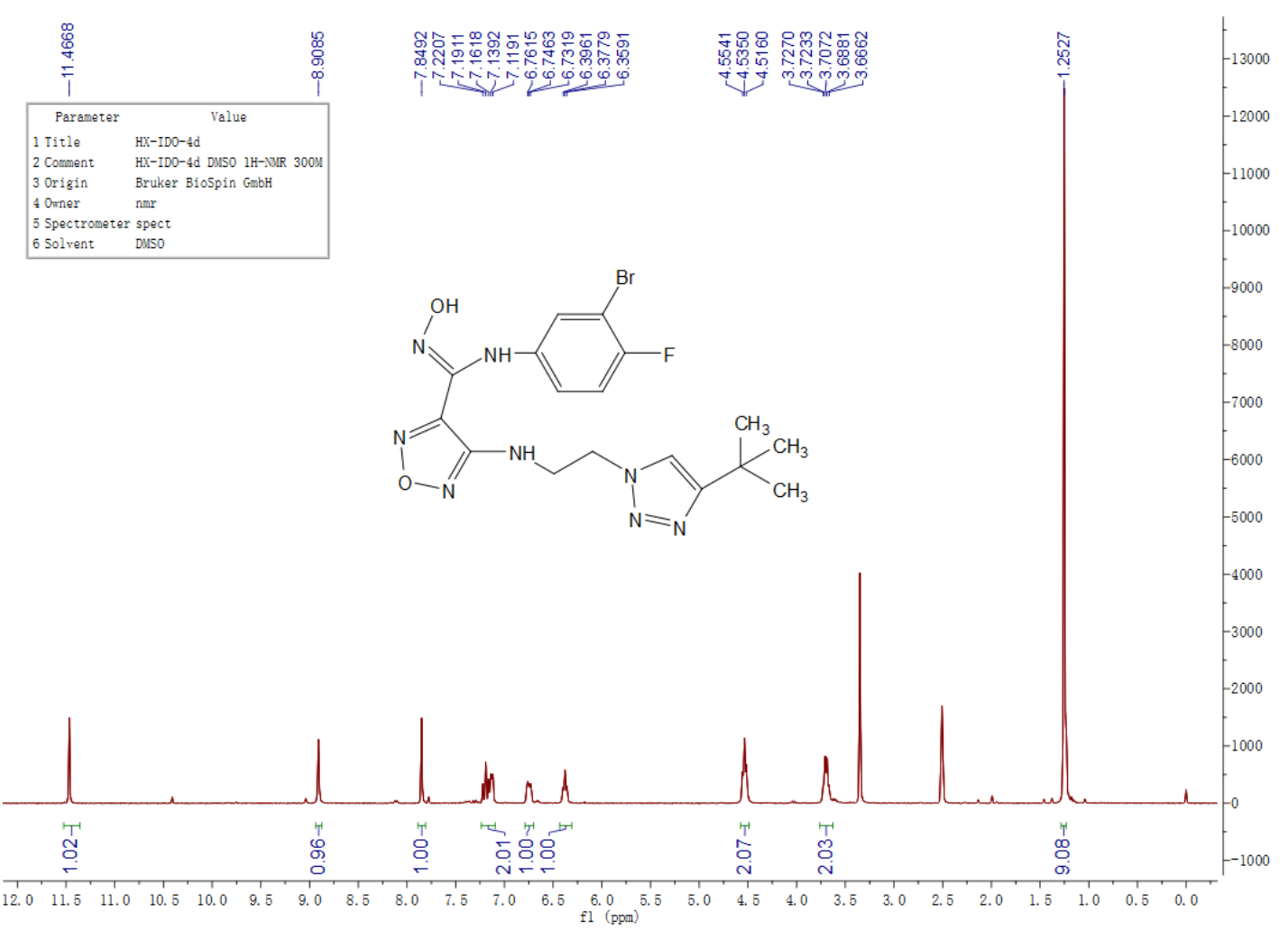

${ }^{13} \mathrm{C}$ NMR of $4 d$
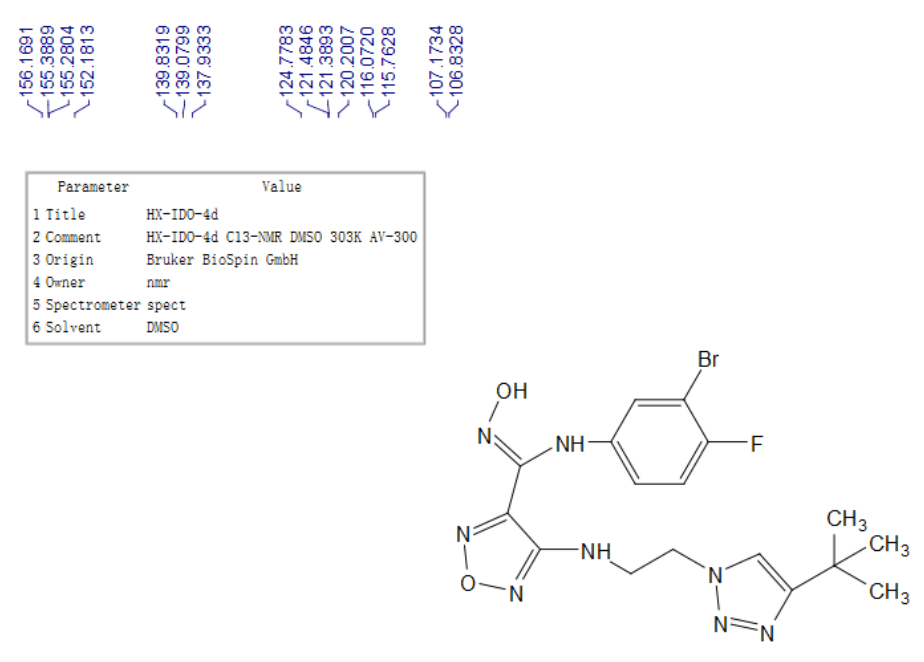

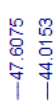

్ㅐㅇㅛ

$-200$

6 Solvent DNSO

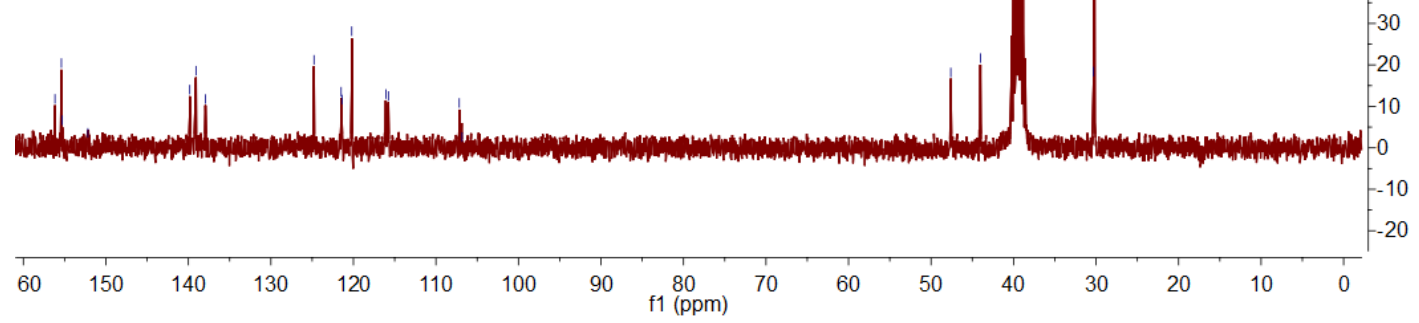




\section{${ }^{1} \mathrm{H}$ NMR of $4 \mathbf{e}$}
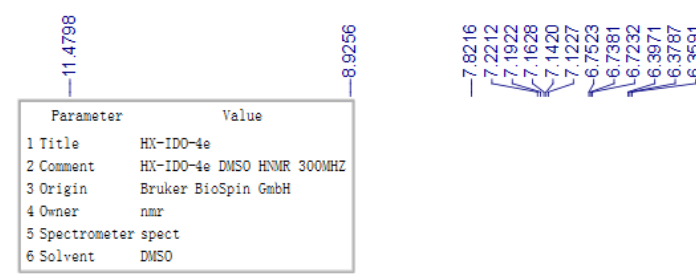

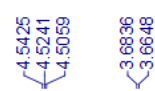
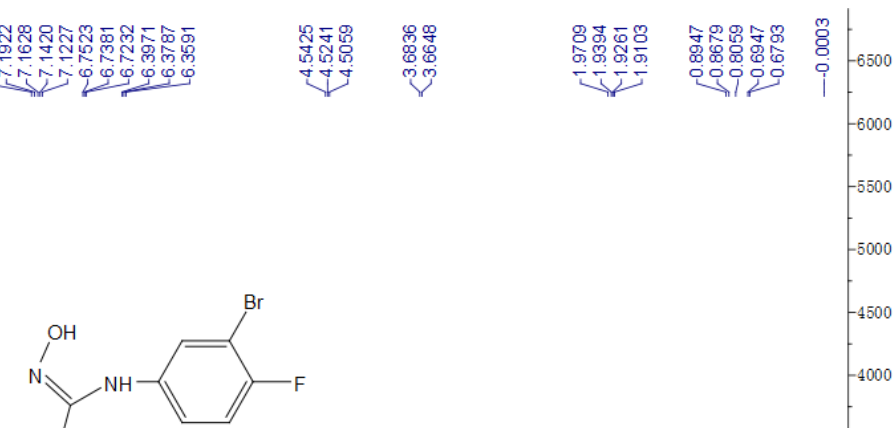

$-4500$
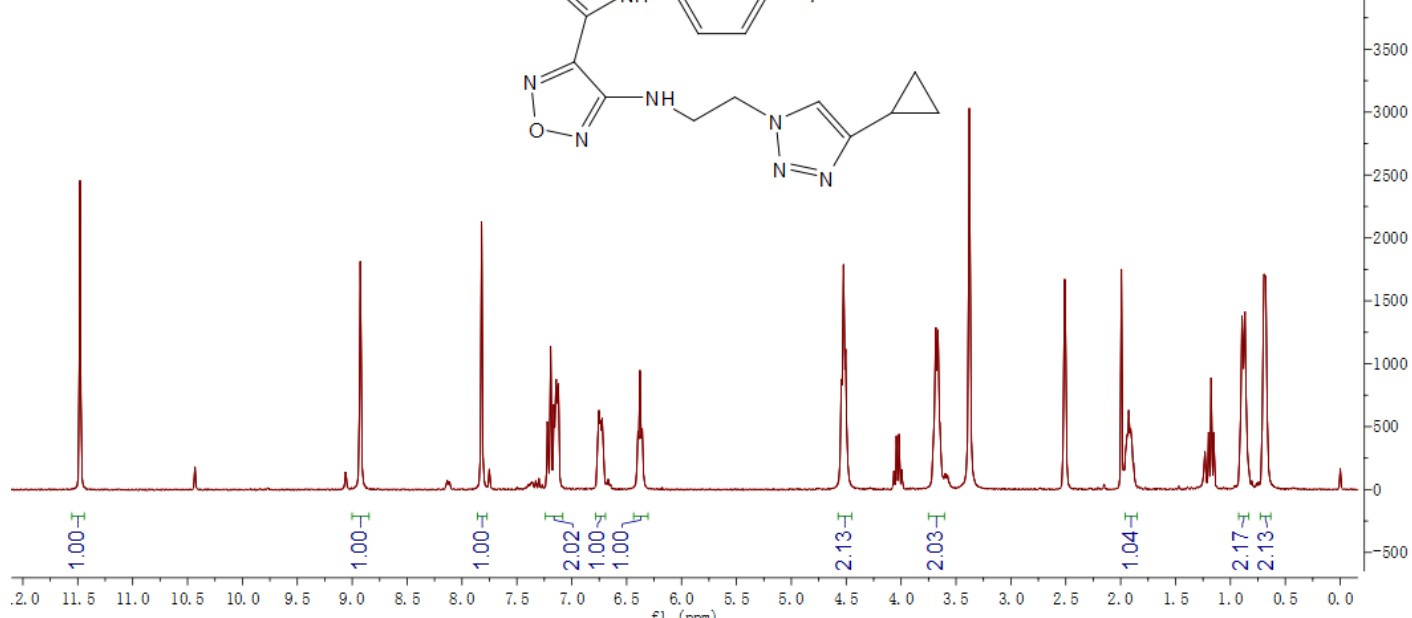

${ }^{13} \mathrm{C}$ NMR of $4 \mathbf{e}$
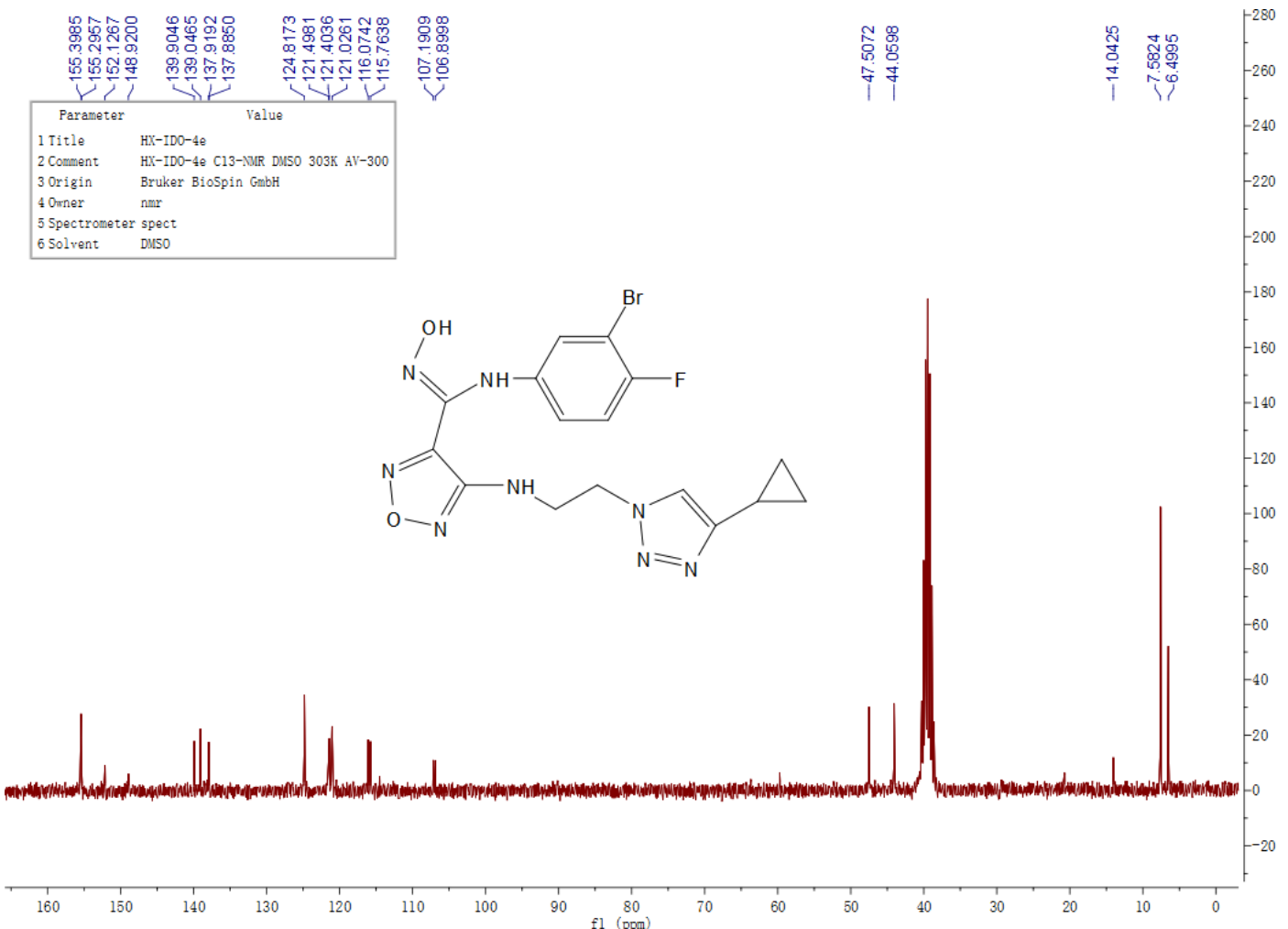
${ }^{1} \mathrm{H}$ NMR of $\mathbf{4 f}$

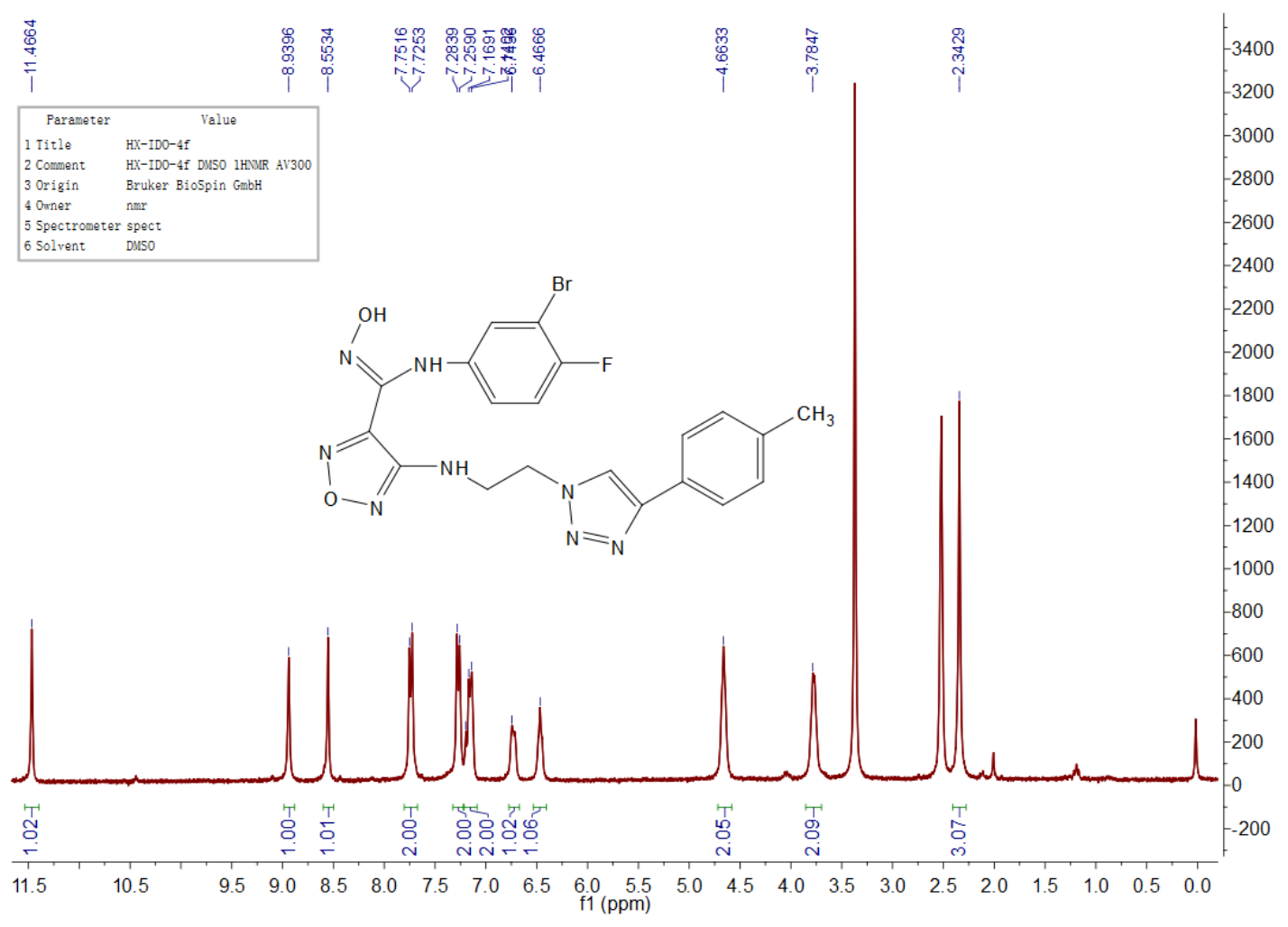

${ }^{13} \mathrm{C}$ NMR of $\mathbf{4 f}$
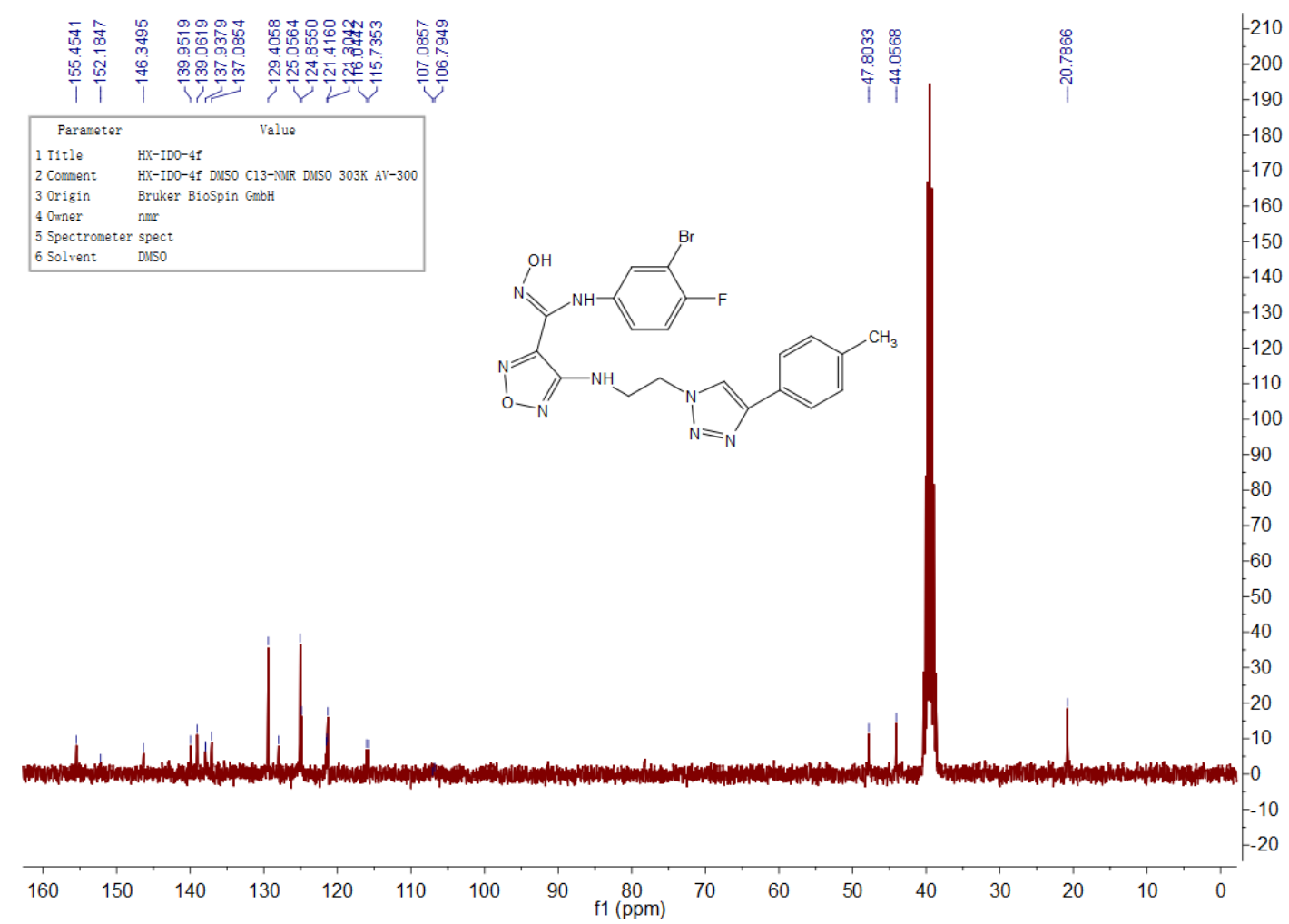
${ }^{1} \mathrm{H}$ NMR of $\mathbf{4 g}$

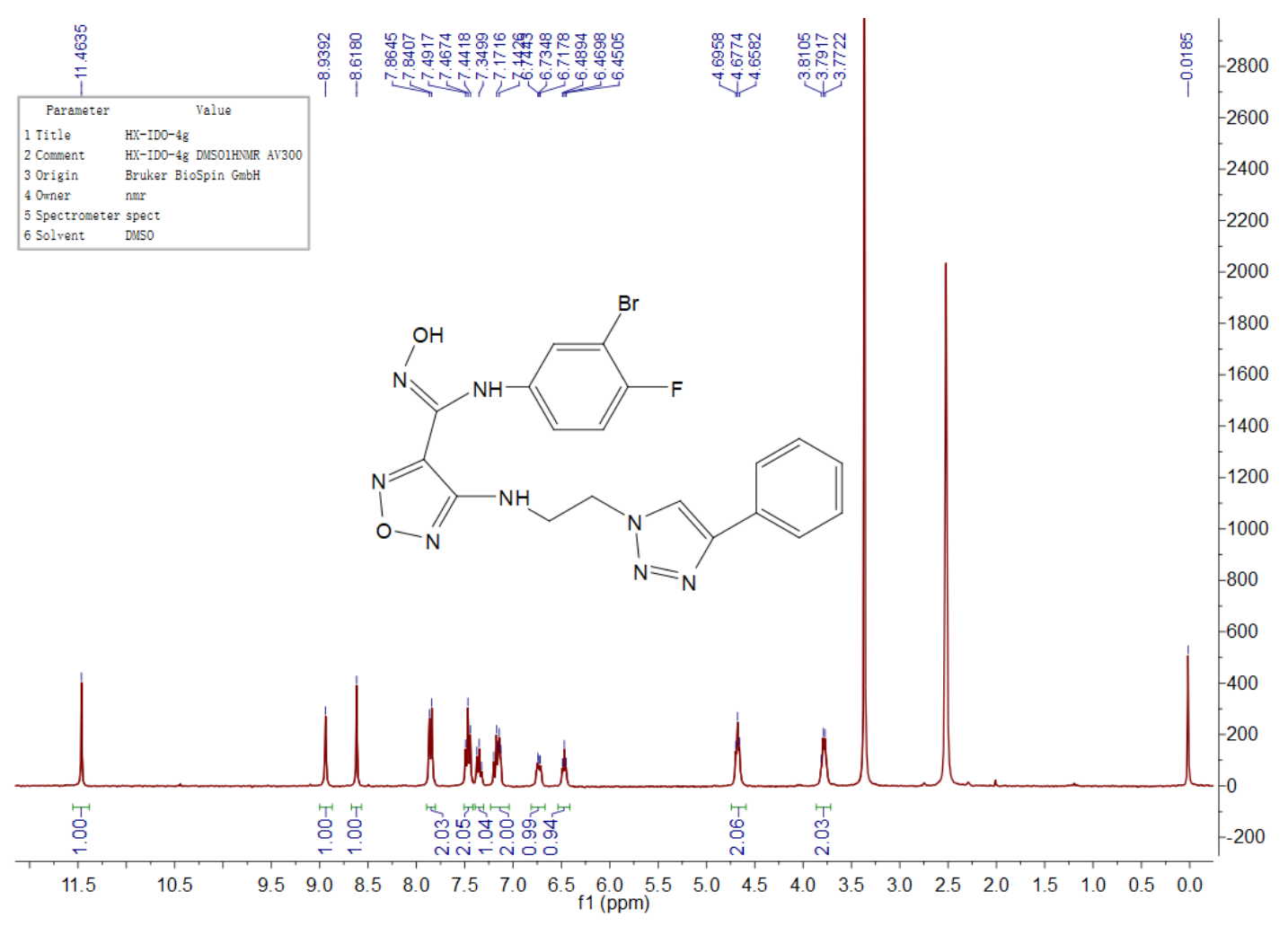

${ }^{13} \mathrm{C}$ NMR of $\mathbf{4 g}$

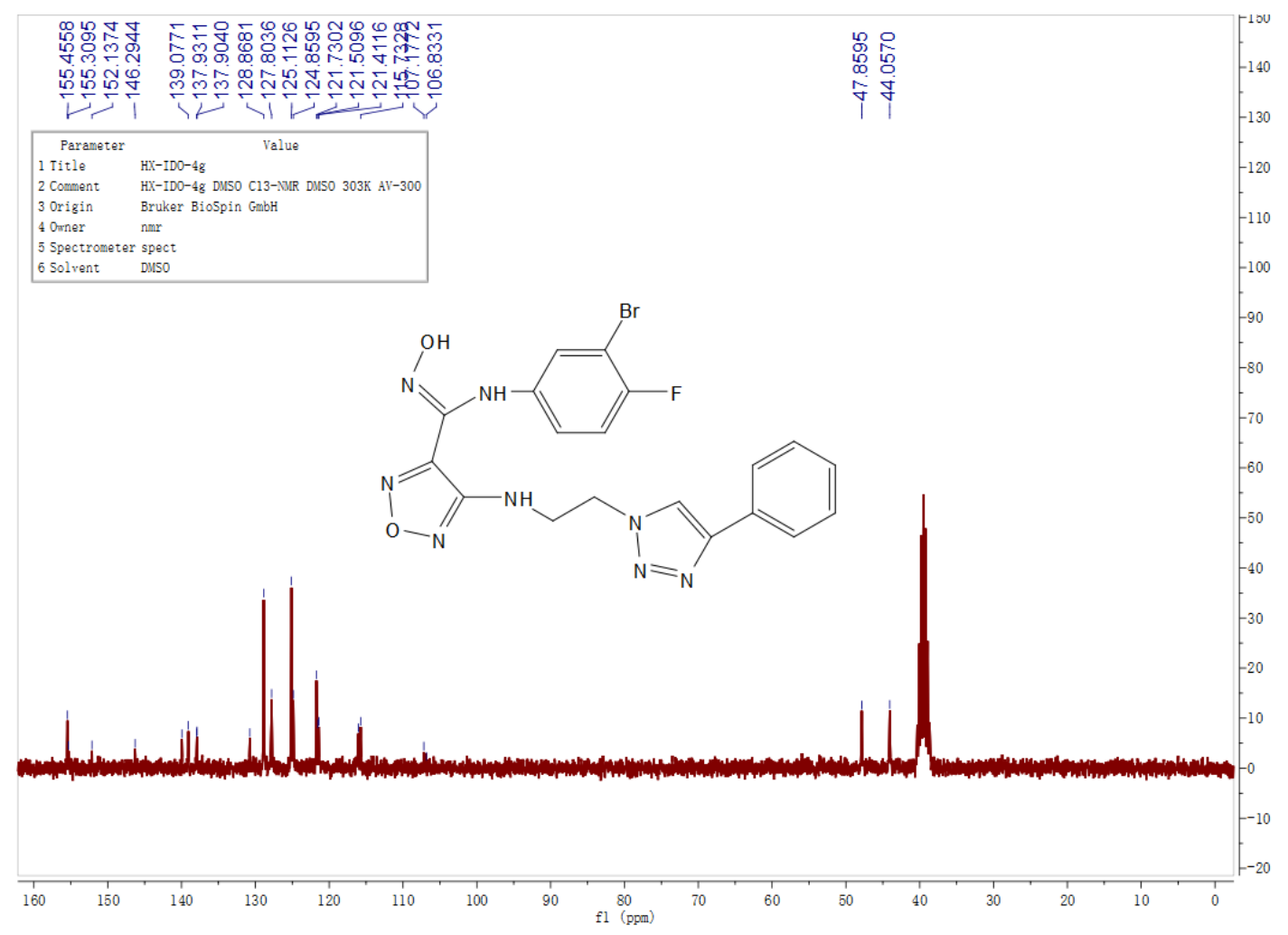


${ }^{1} \mathrm{H}$ NMR of $\mathbf{4 h}$

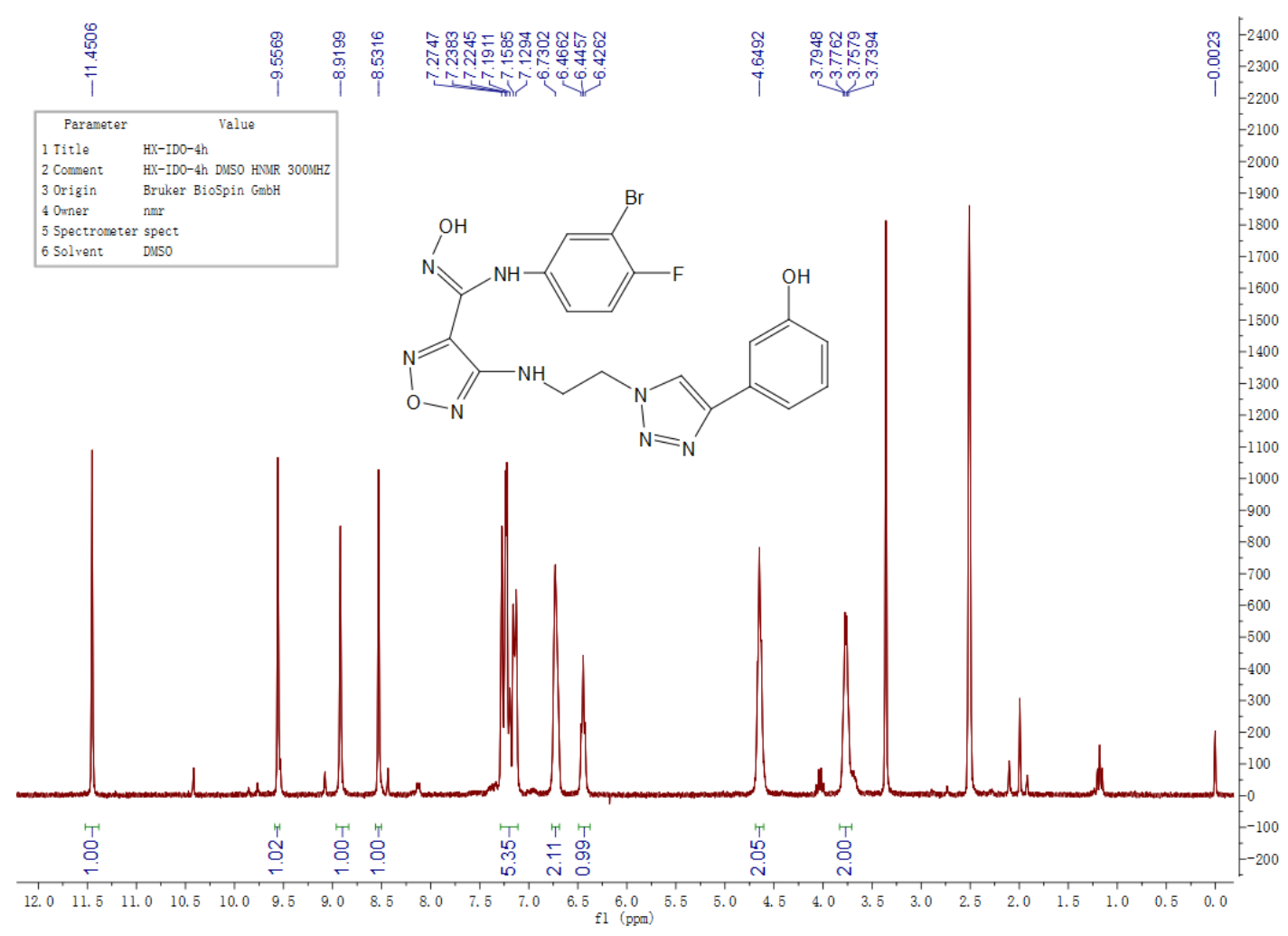

${ }^{13} \mathrm{C}$ NMR of $\mathbf{4 h}$

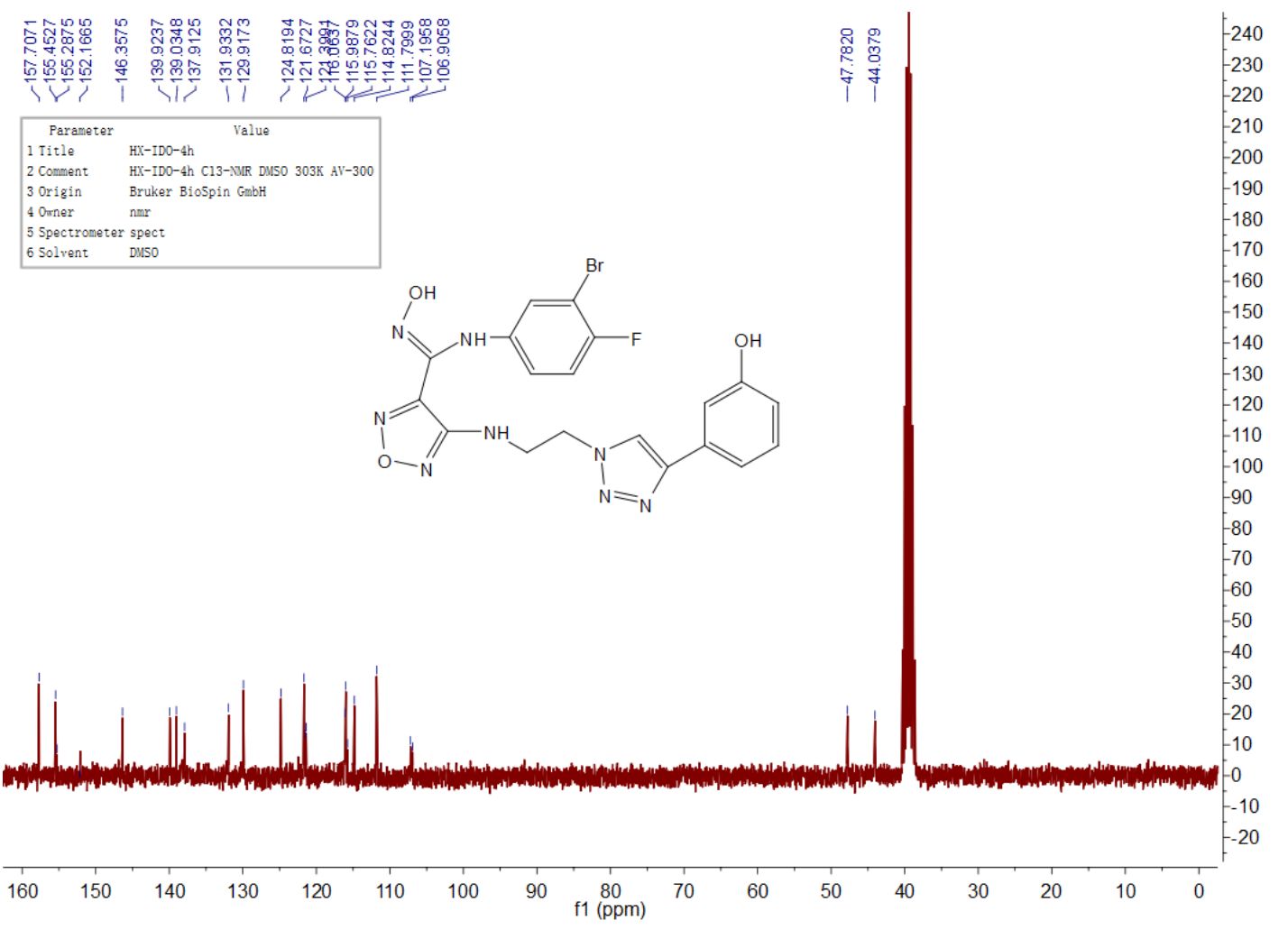


${ }^{1} \mathrm{H}$ NMR of $4 \mathbf{i}$

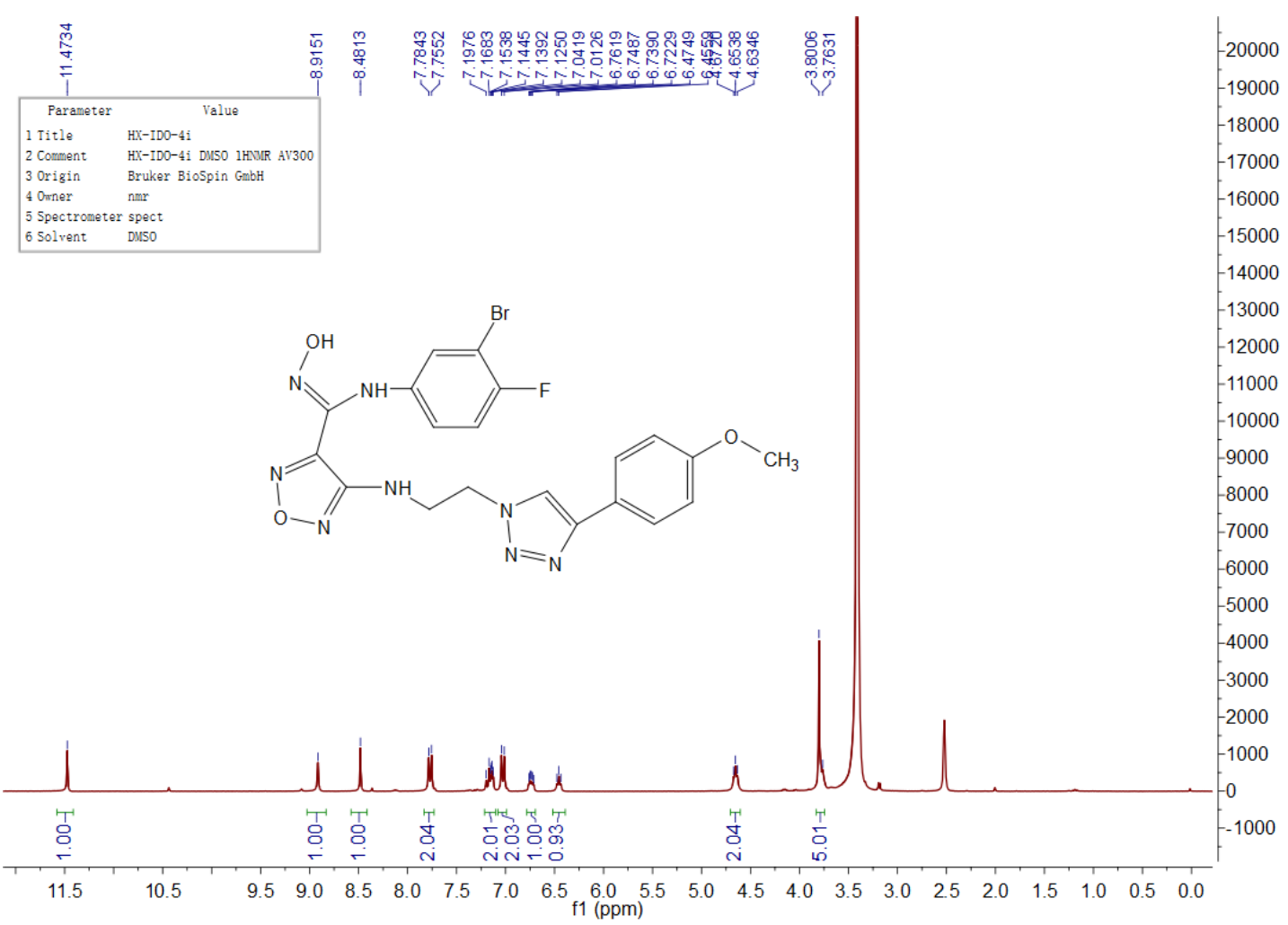

${ }^{13} \mathrm{C}$ NMR of $4 \mathbf{i}$
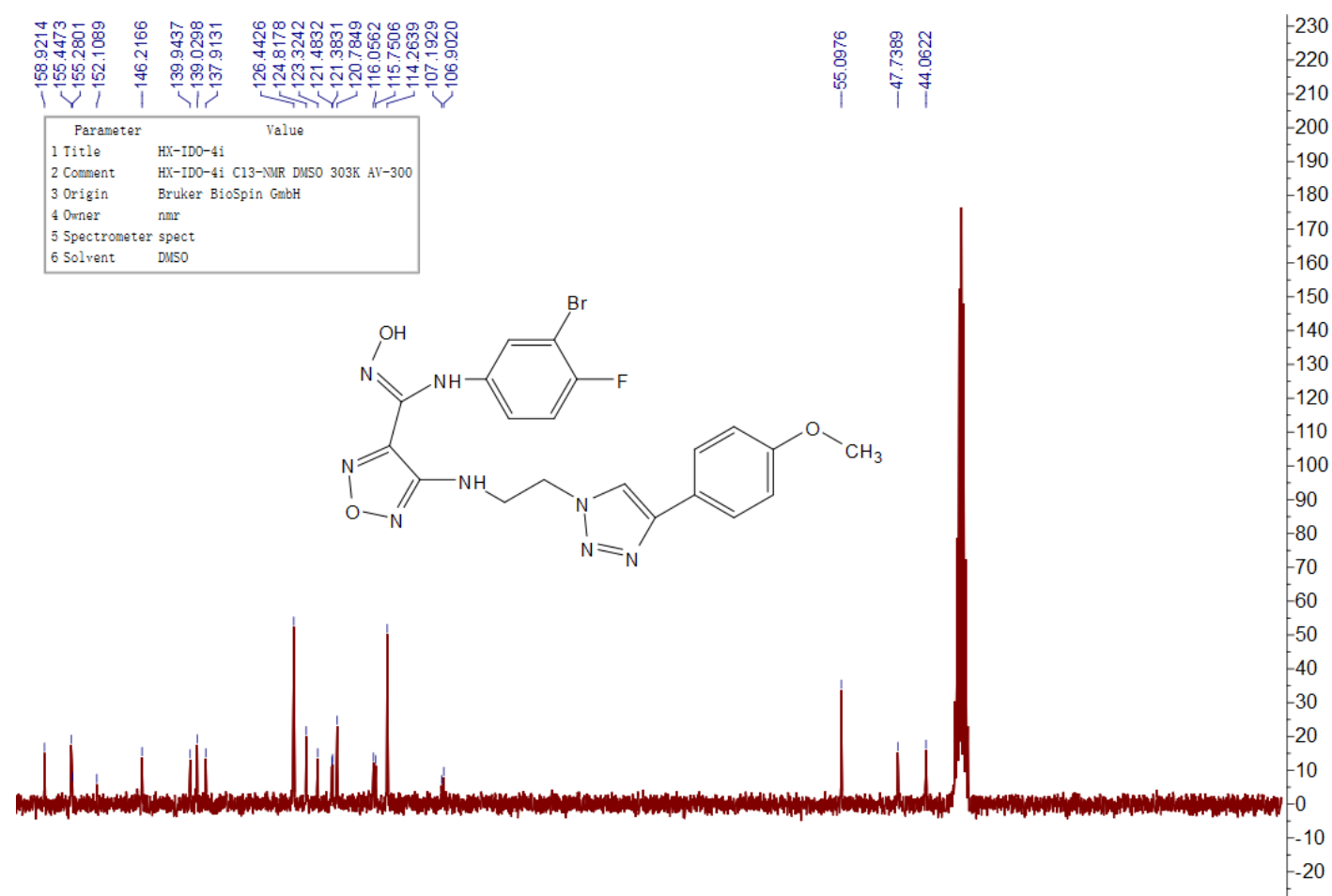

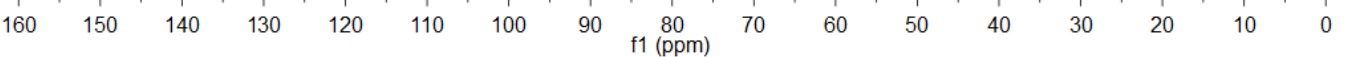


${ }^{41} \mathrm{H}$ NMR of $\mathbf{4 j}$

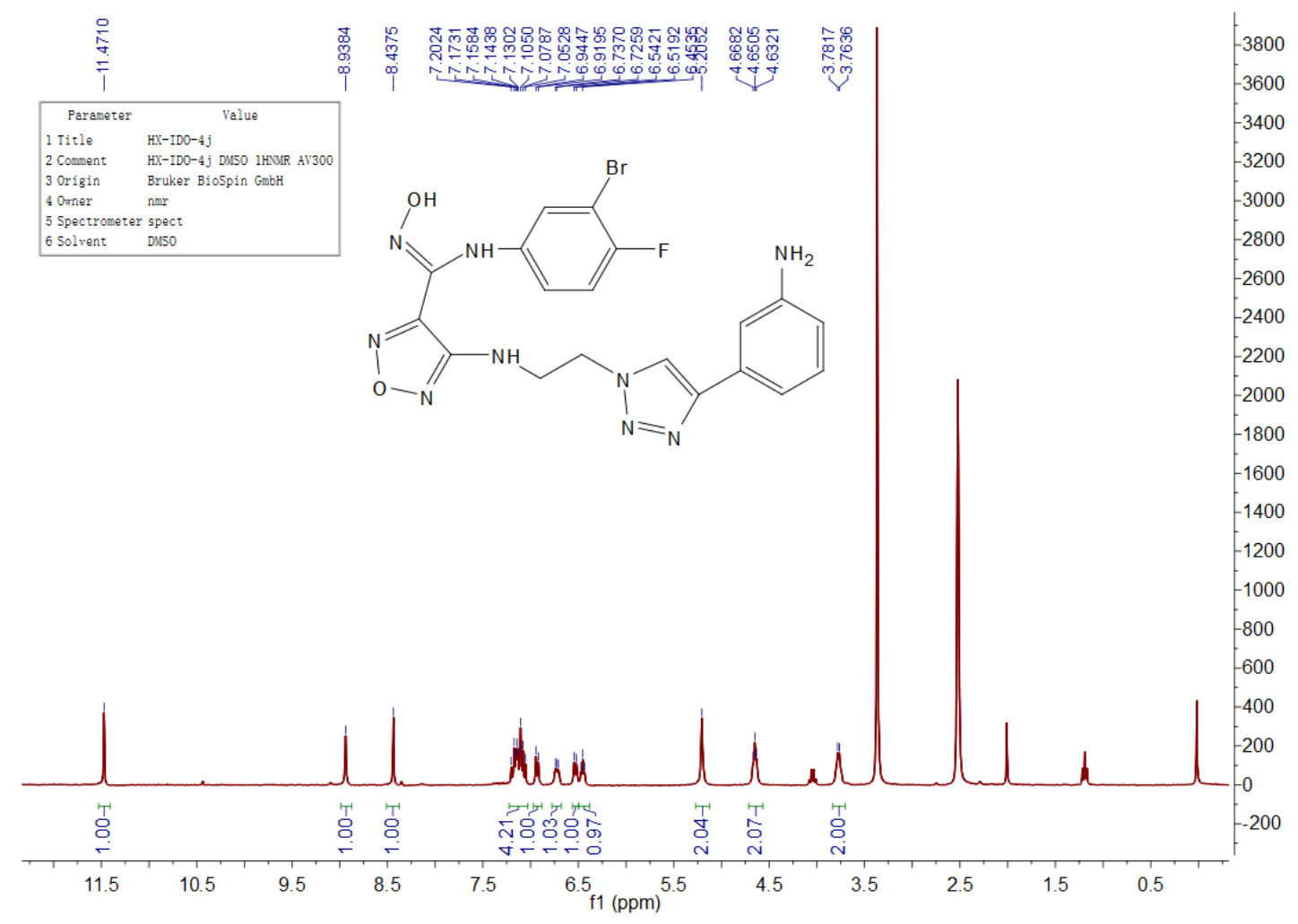

${ }^{13} \mathrm{C}$ NMR of $4 \mathbf{j}$

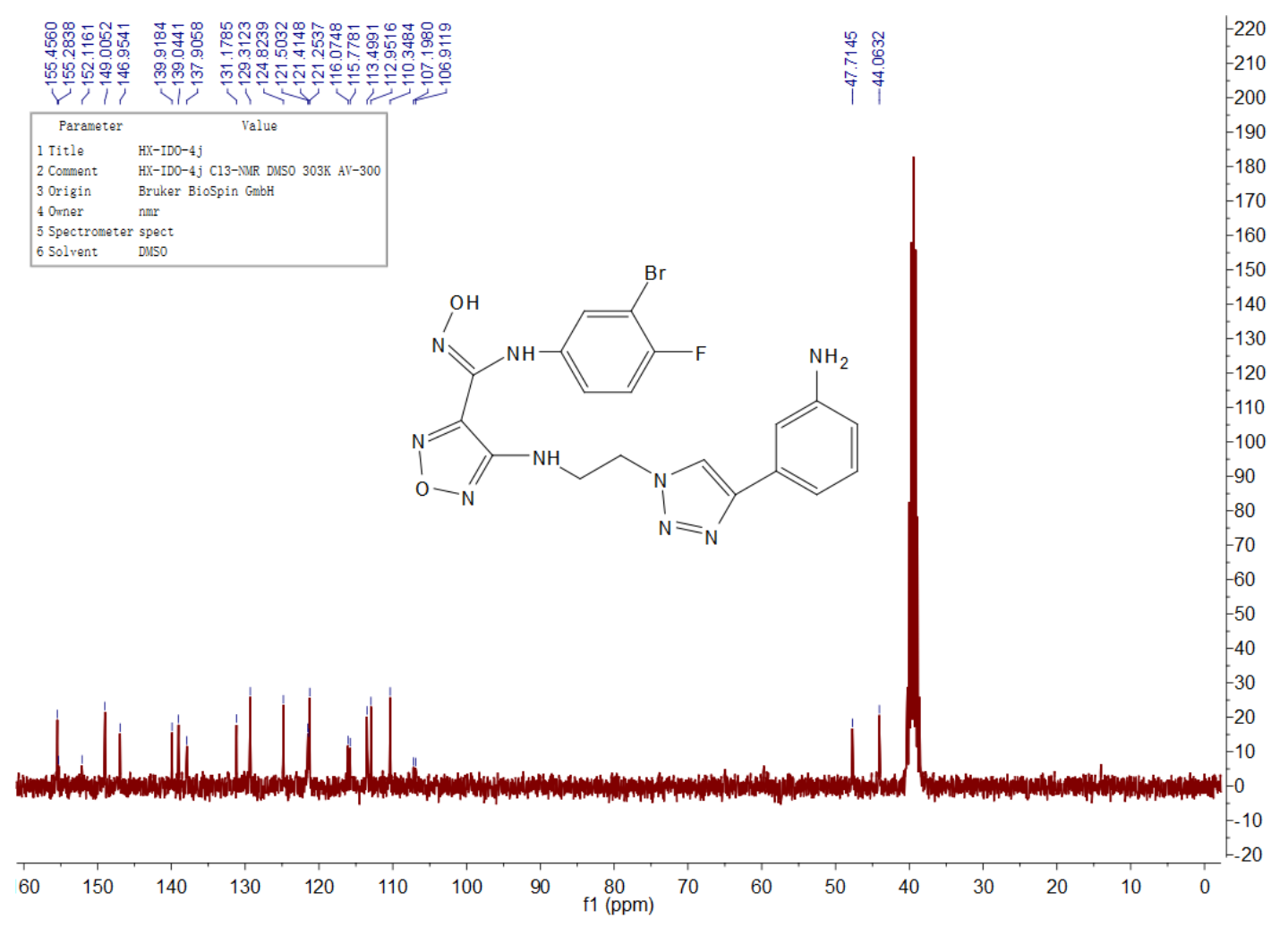


${ }^{1} \mathrm{H}$ NMR of $\mathbf{4 k}$

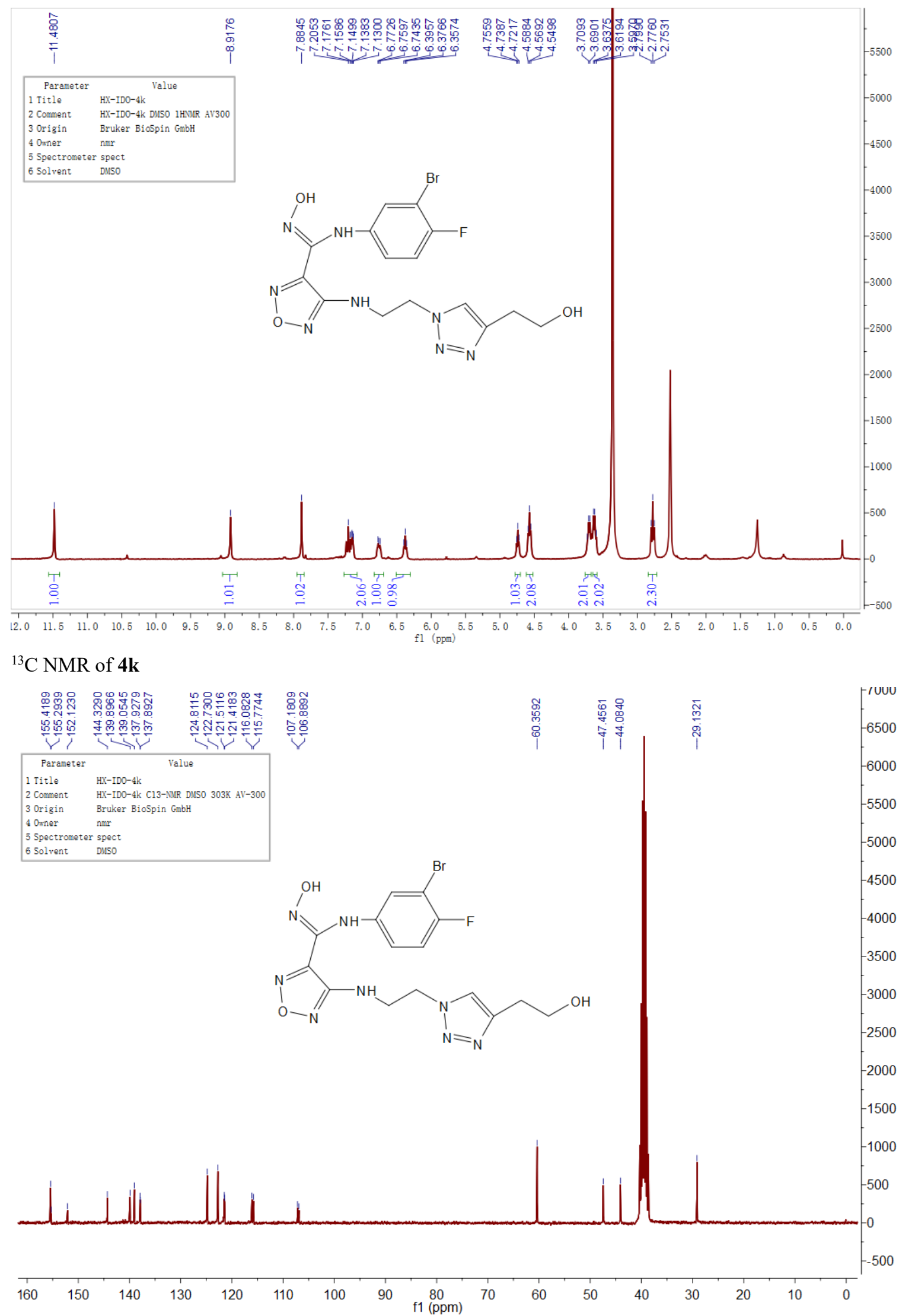


${ }^{1} \mathrm{H}$ NMR of 41

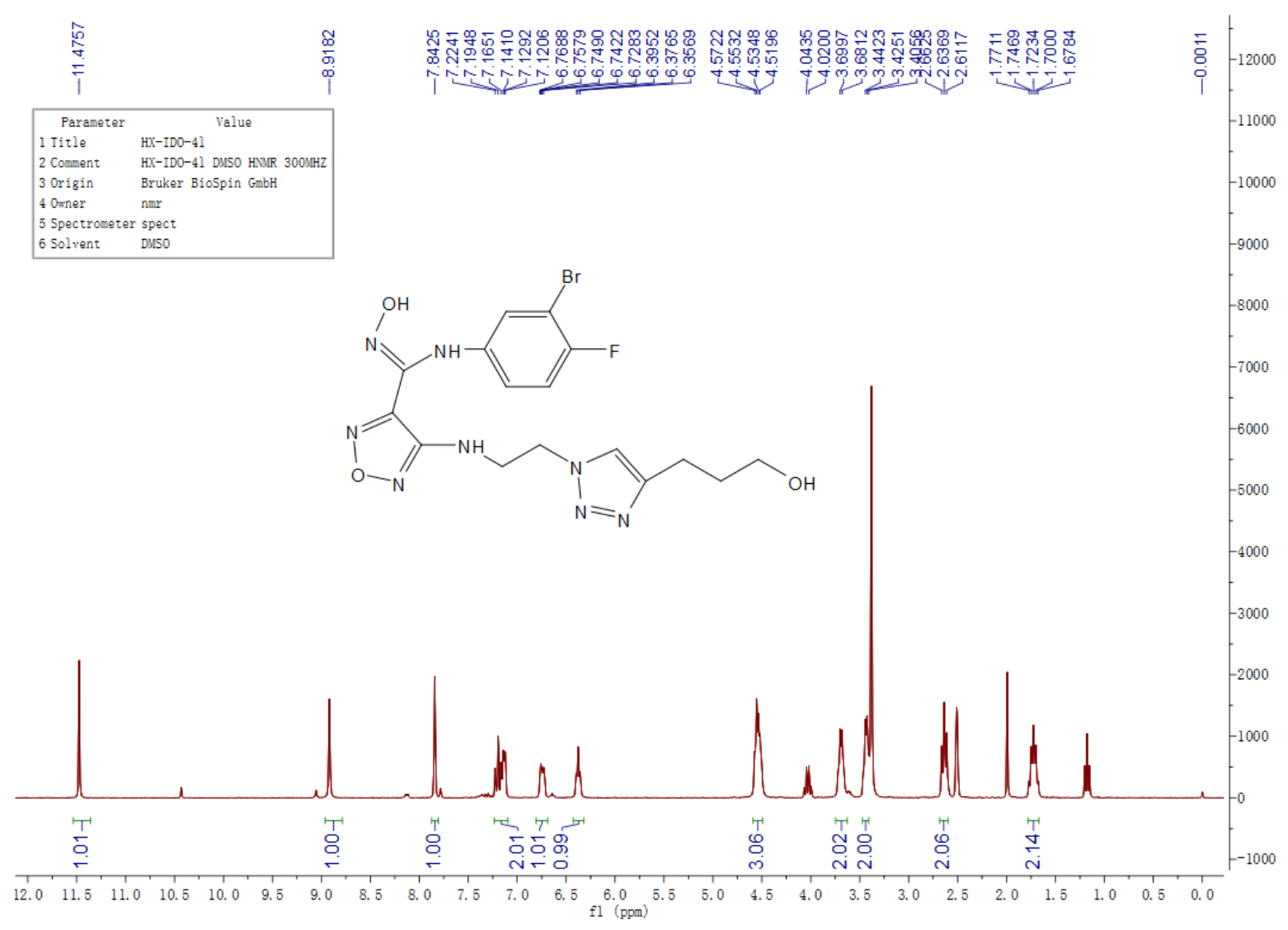

${ }^{13} \mathrm{C}$ NMR of 41

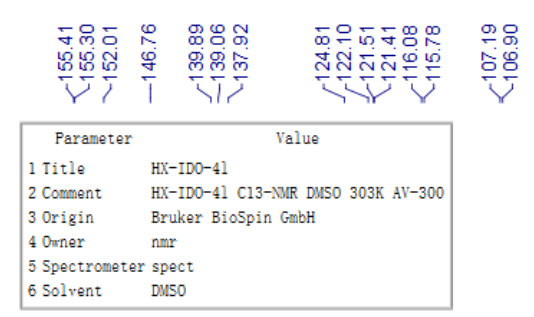

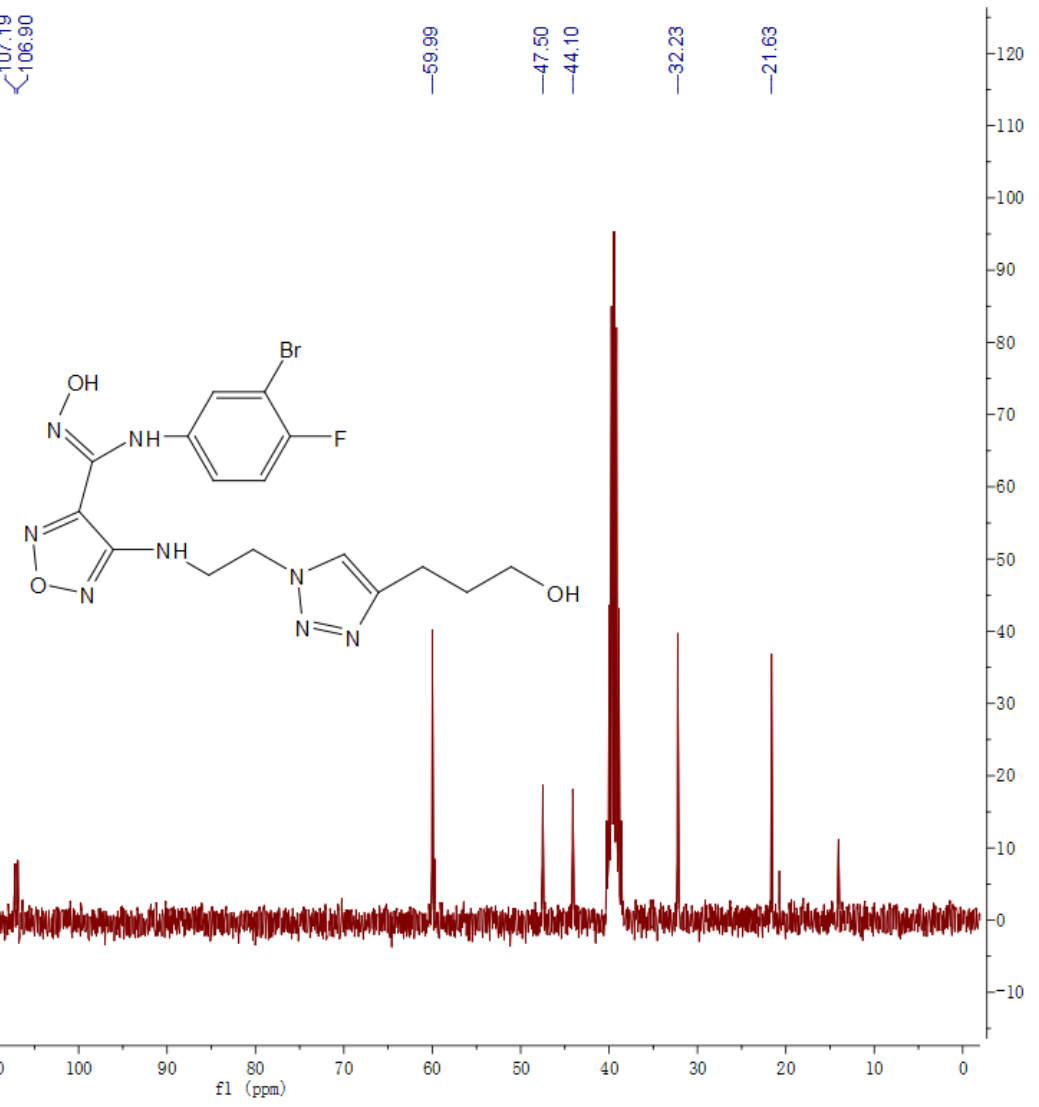


${ }^{1} \mathrm{H}$ NMR of $\mathbf{4 m}$

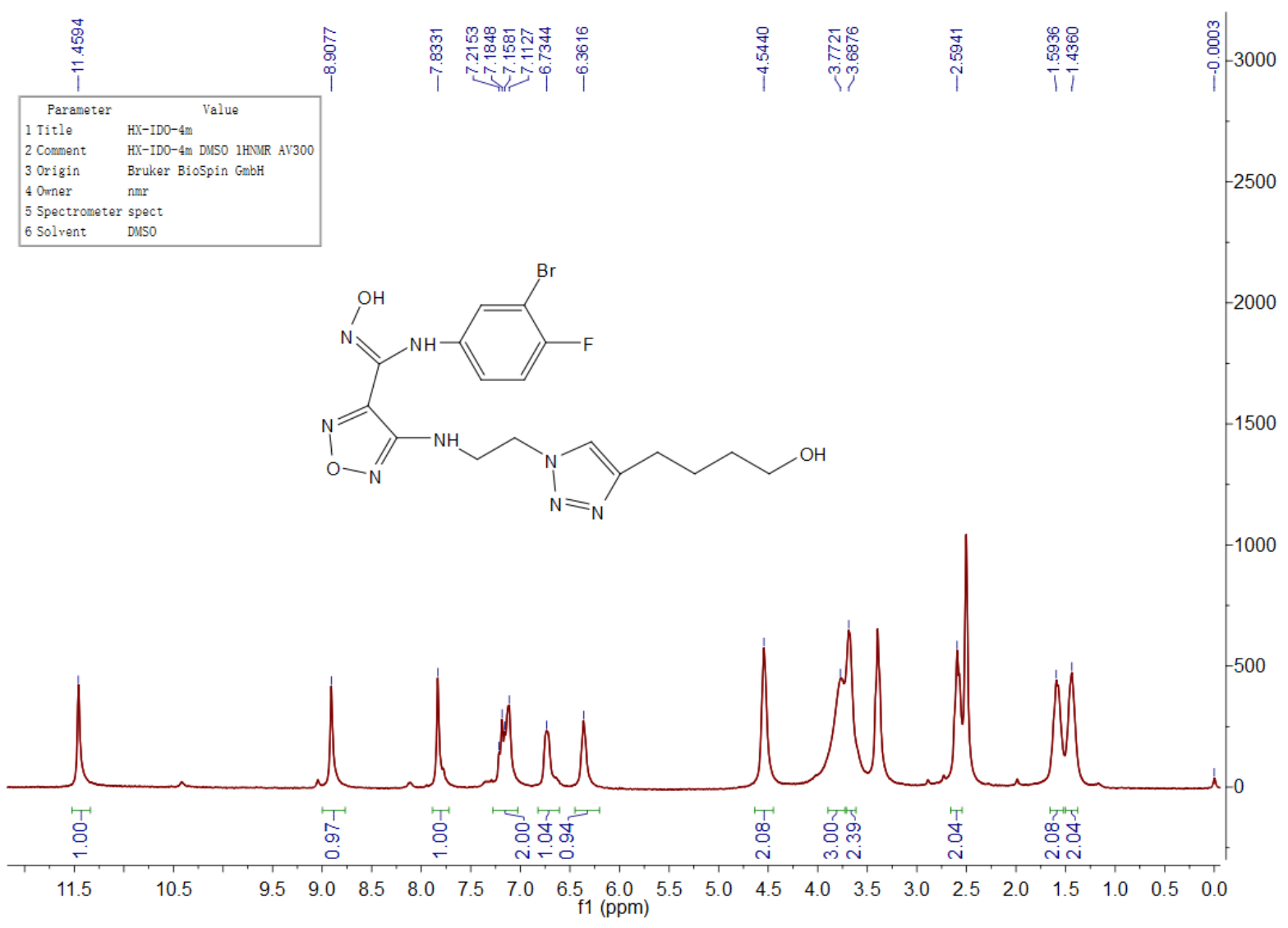

${ }^{13} \mathrm{C}$ NMR of $4 \mathrm{~m}$

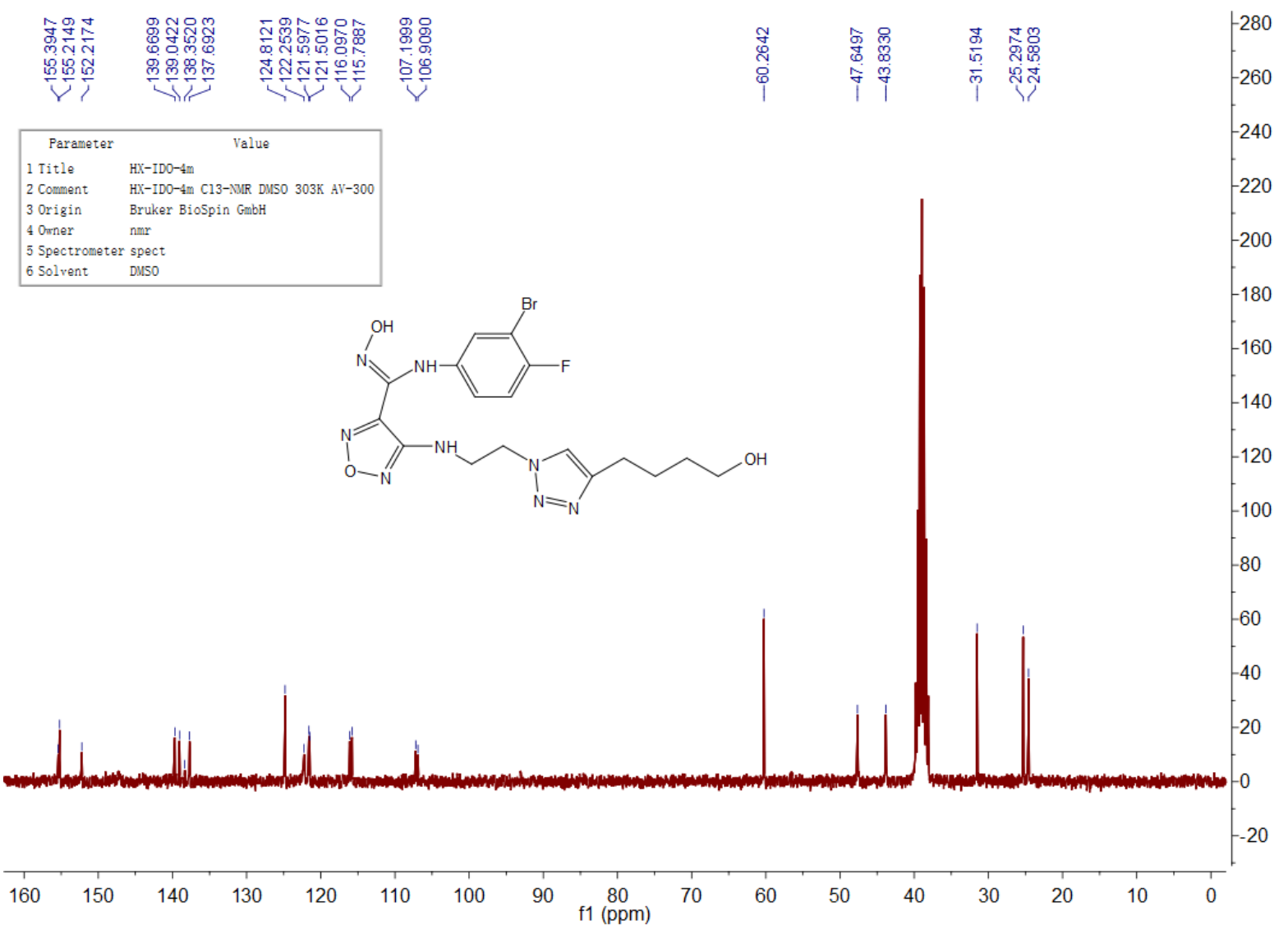


${ }^{1} \mathrm{H}$ NMR of 4 n

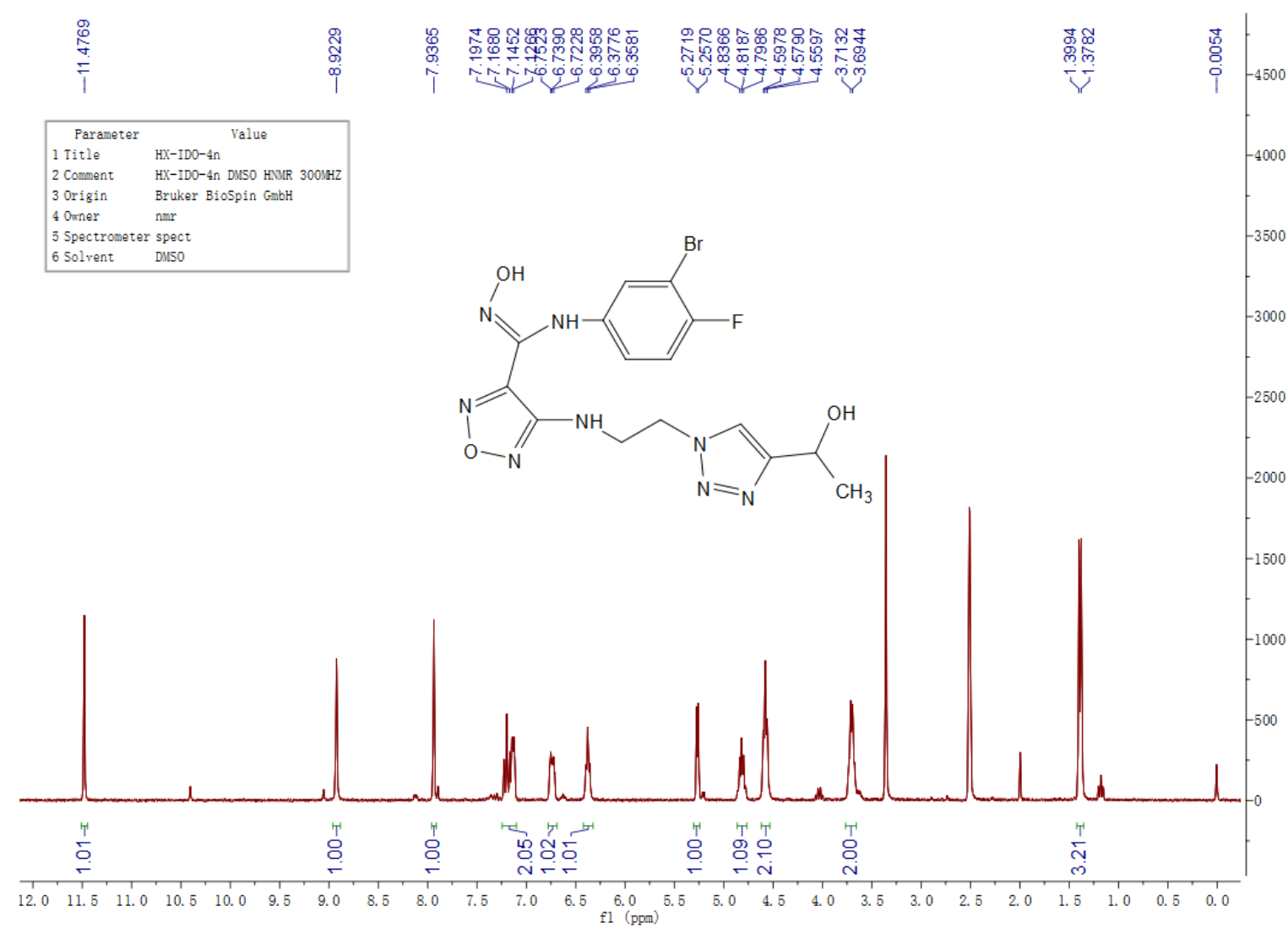

${ }^{13} \mathrm{C}$ NMR of $4 n$

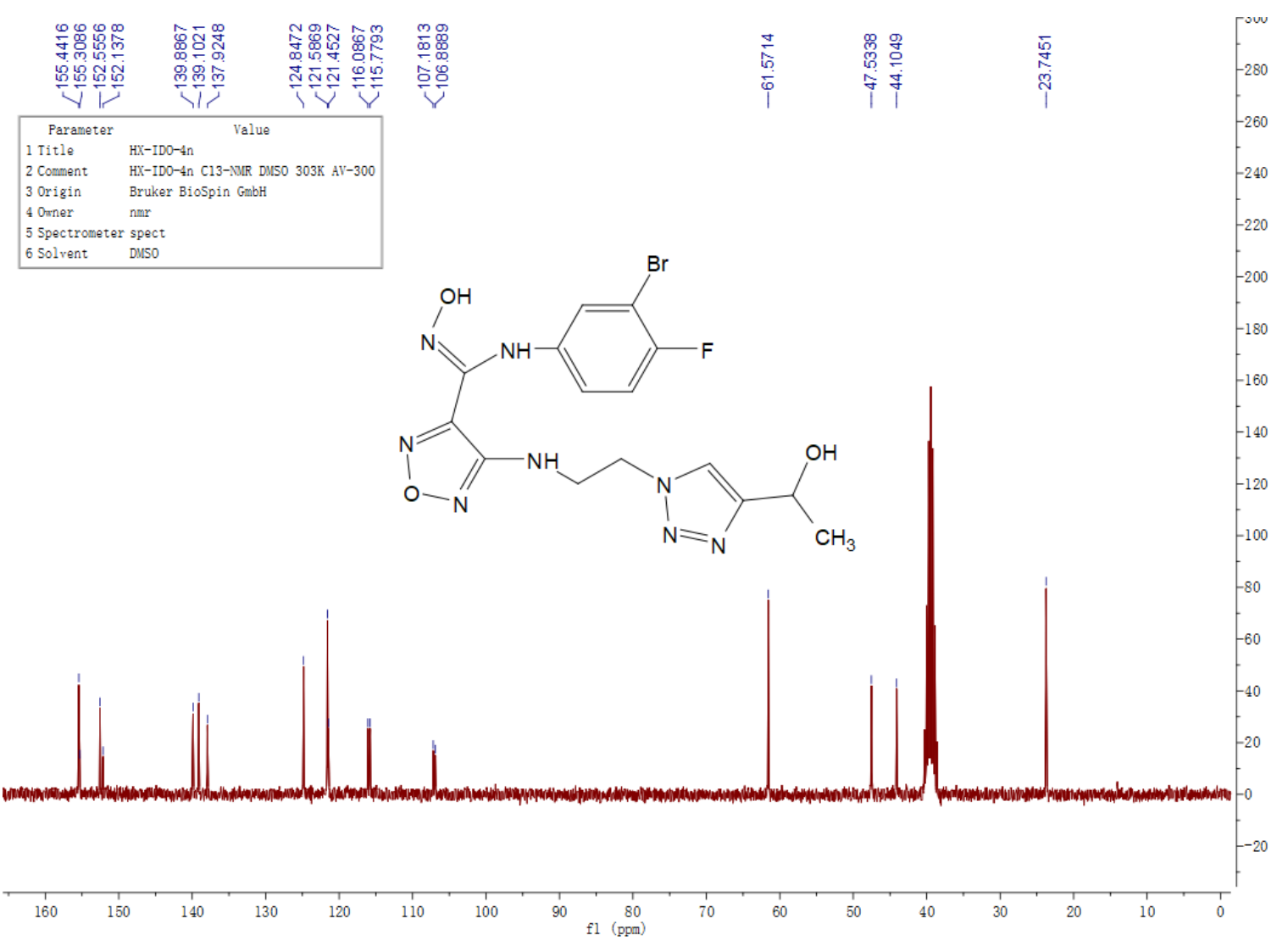


${ }^{1} \mathrm{H}$ NMR of 40

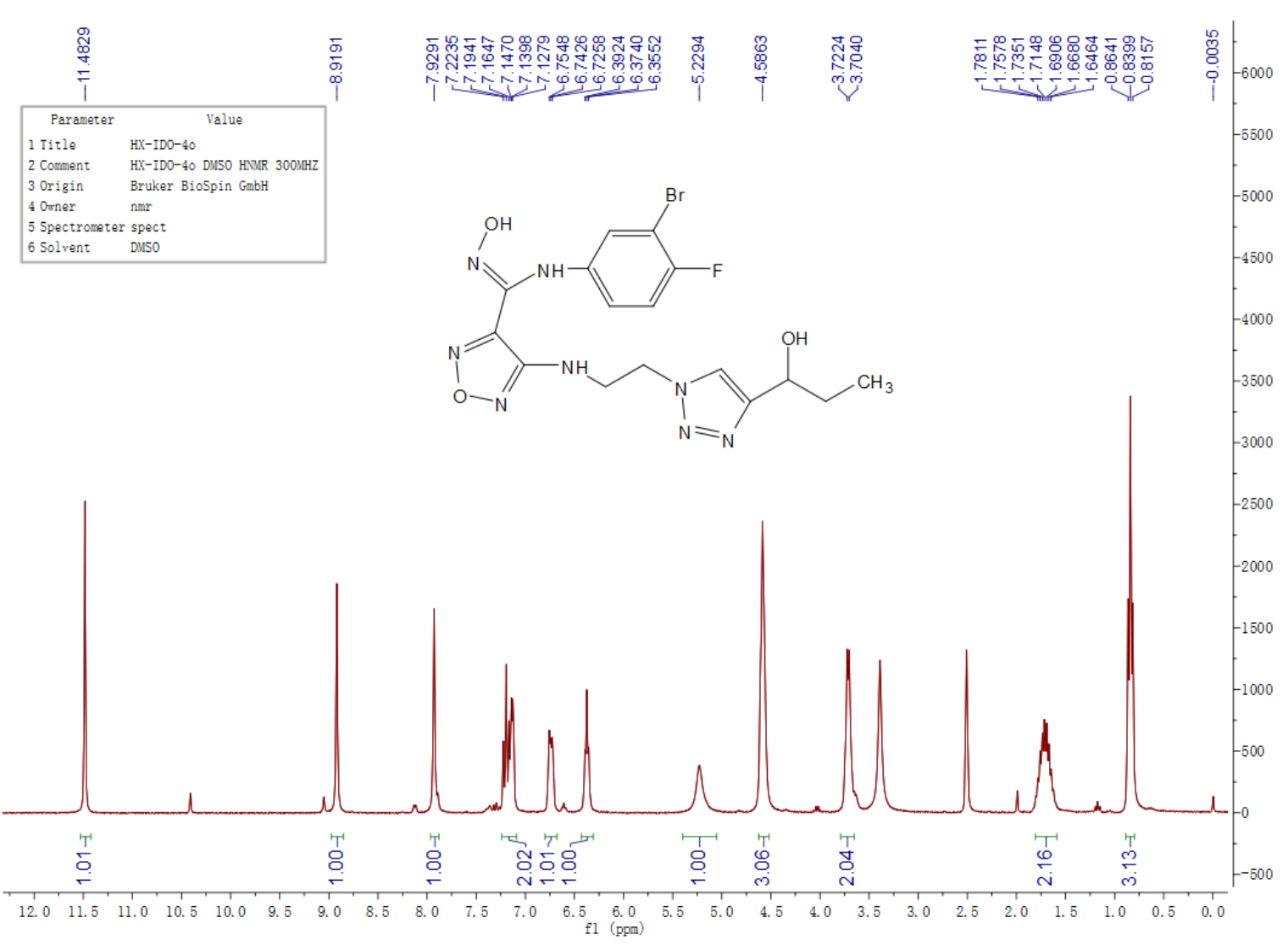

${ }^{13} \mathrm{C}$ NMR of 40

\begin{tabular}{|c|c|c|}
\hline 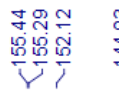 & 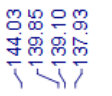 & 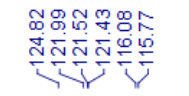 \\
\hline Parameter & & Value \\
\hline 1 Title & $\mathrm{HX}-\mathrm{IDO}-40$ & \\
\hline 2 Comment & $\mathrm{HX}-\mathrm{IDO}-40$ & - IMR DMSO $303 \mathrm{~K} \mathrm{AV}-300$ \\
\hline 3 origin & Bruker Bio & $\mathrm{n}$ GmbH \\
\hline 4 Owner & $\mathrm{nmr}$ & \\
\hline \multicolumn{3}{|c|}{5 spectrometer spect } \\
\hline 6 Solvent & DMSO & \\
\hline
\end{tabular}<smiles>CCC(O)c1cn(CCNc2nonc2/C(=N/O)Nc2ccc(F)c(Br)c2)nn1</smiles>

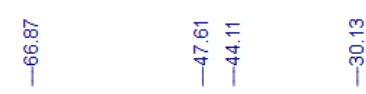

$\stackrel{9}{\circ}$

$-240$

\section{.}


${ }^{1} \mathrm{H}$ NMR of $\mathbf{4 p}$

\begin{tabular}{|ll|}
\hline \multicolumn{1}{|c|}{ Parameter } & \multicolumn{1}{c|}{ Value } \\
1 Title & HX-IDO-4p \\
2 Comment & HXX-IDO-4p DMSO HNR 300NHZ \\
3 Origin & Bruker BioSpin GmbH \\
4 Orner & nmm \\
5 Spectrometer spect \\
6 Solvent & DMSO \\
\hline
\end{tabular}
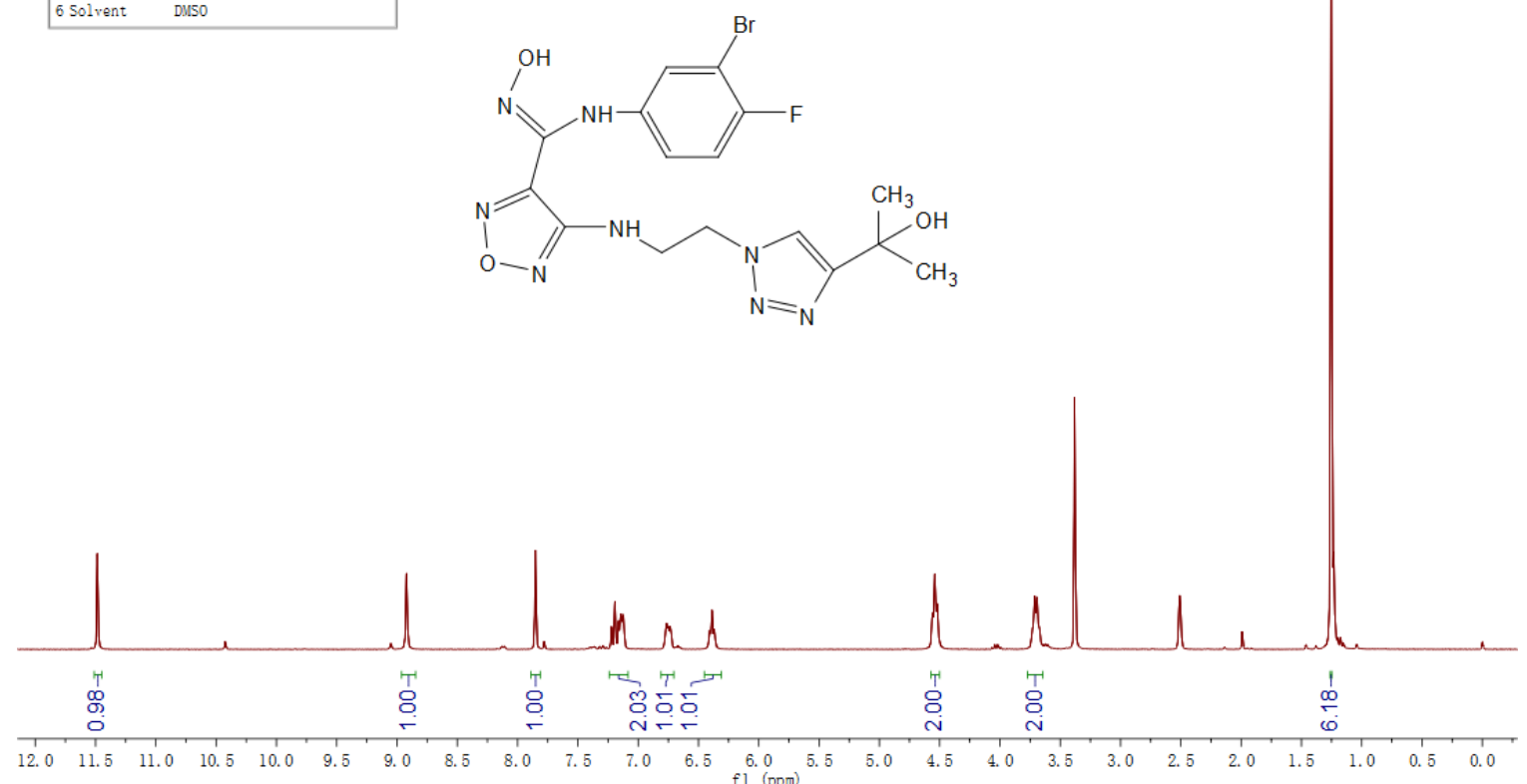

${ }^{13} \mathrm{C}$ NMR of $4 p$
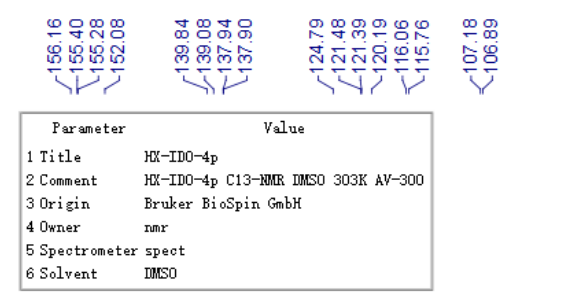

吕

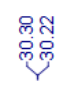

$-300$
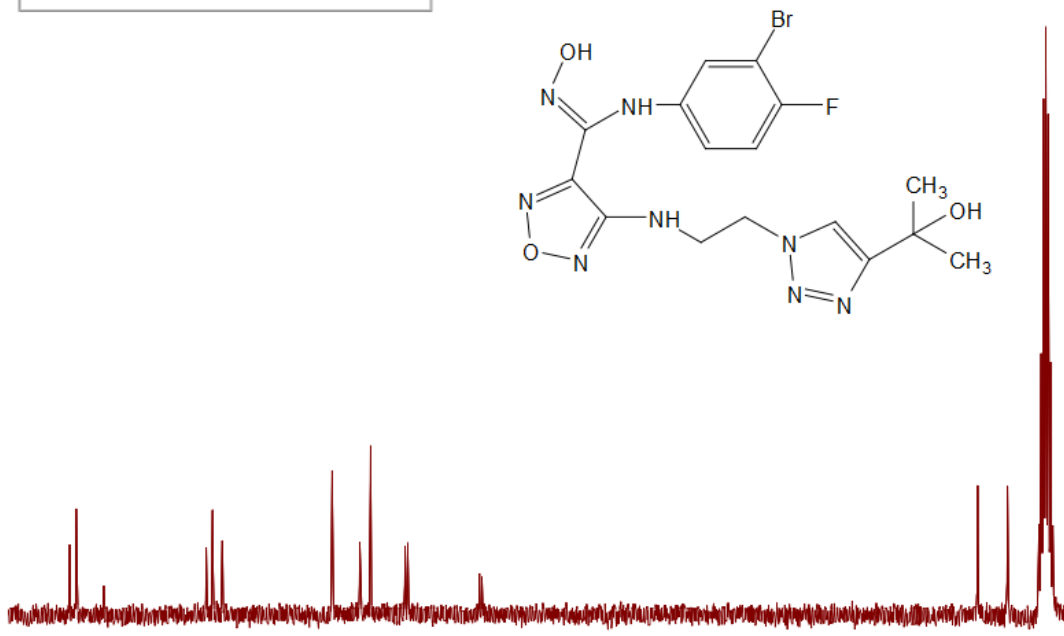

160

150

$140 \quad 130$

120

100

80
$\mathrm{f} 1 \quad(\mathrm{ppm})$ 
${ }^{1} \mathrm{H}$ NMR of $\mathbf{4 q}$

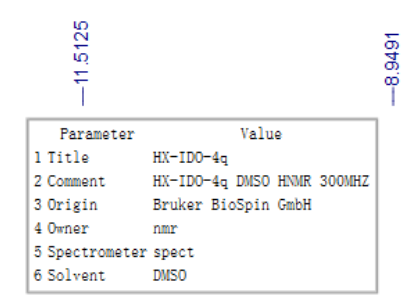

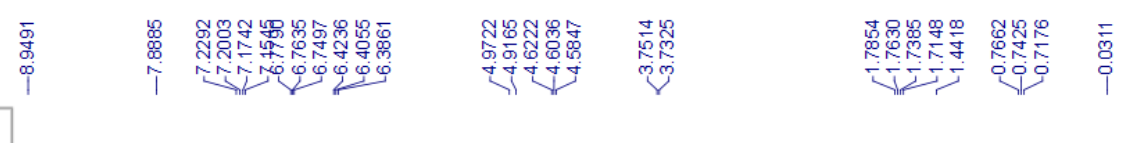<smiles>CCC(C)(O)c1cn(CCNc2nonc2/C(=N/O)Nc2ccc(F)c(Br)c2)nn1</smiles>

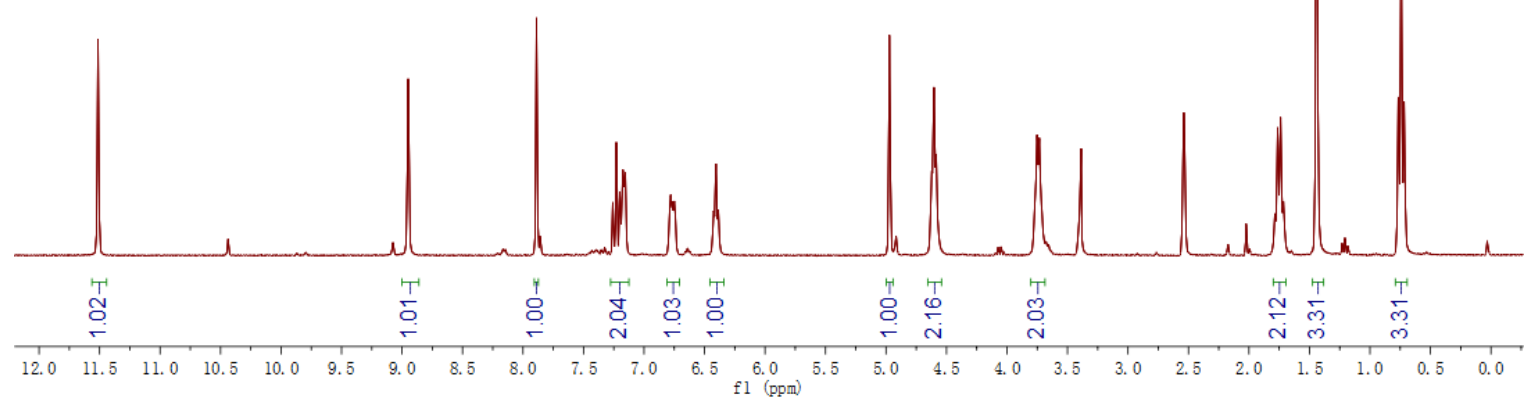

${ }^{13} \mathrm{C}$ NMR of $\mathbf{4 q}$

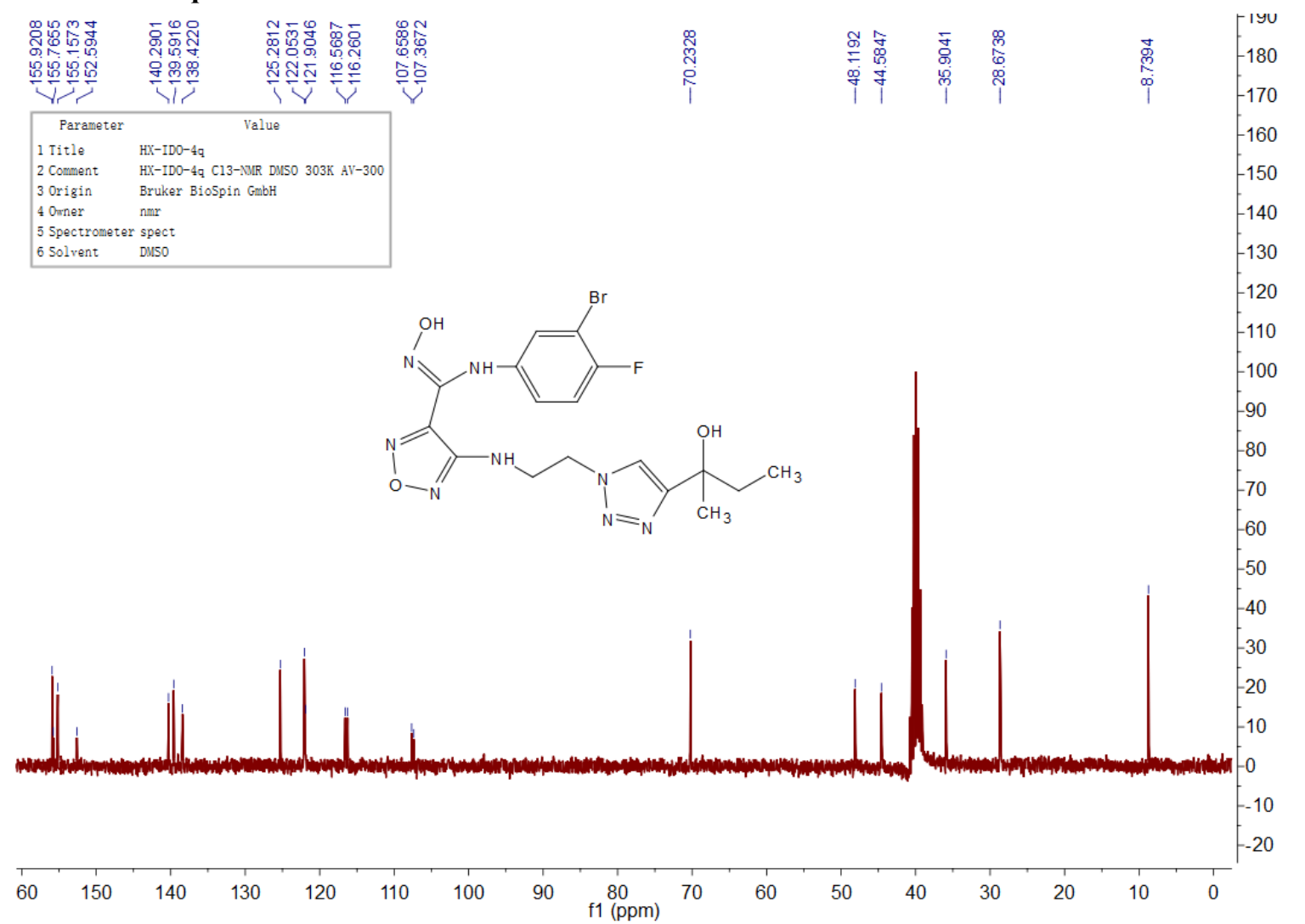


${ }^{1} \mathrm{H}$ NMR of $\mathbf{4 r}$

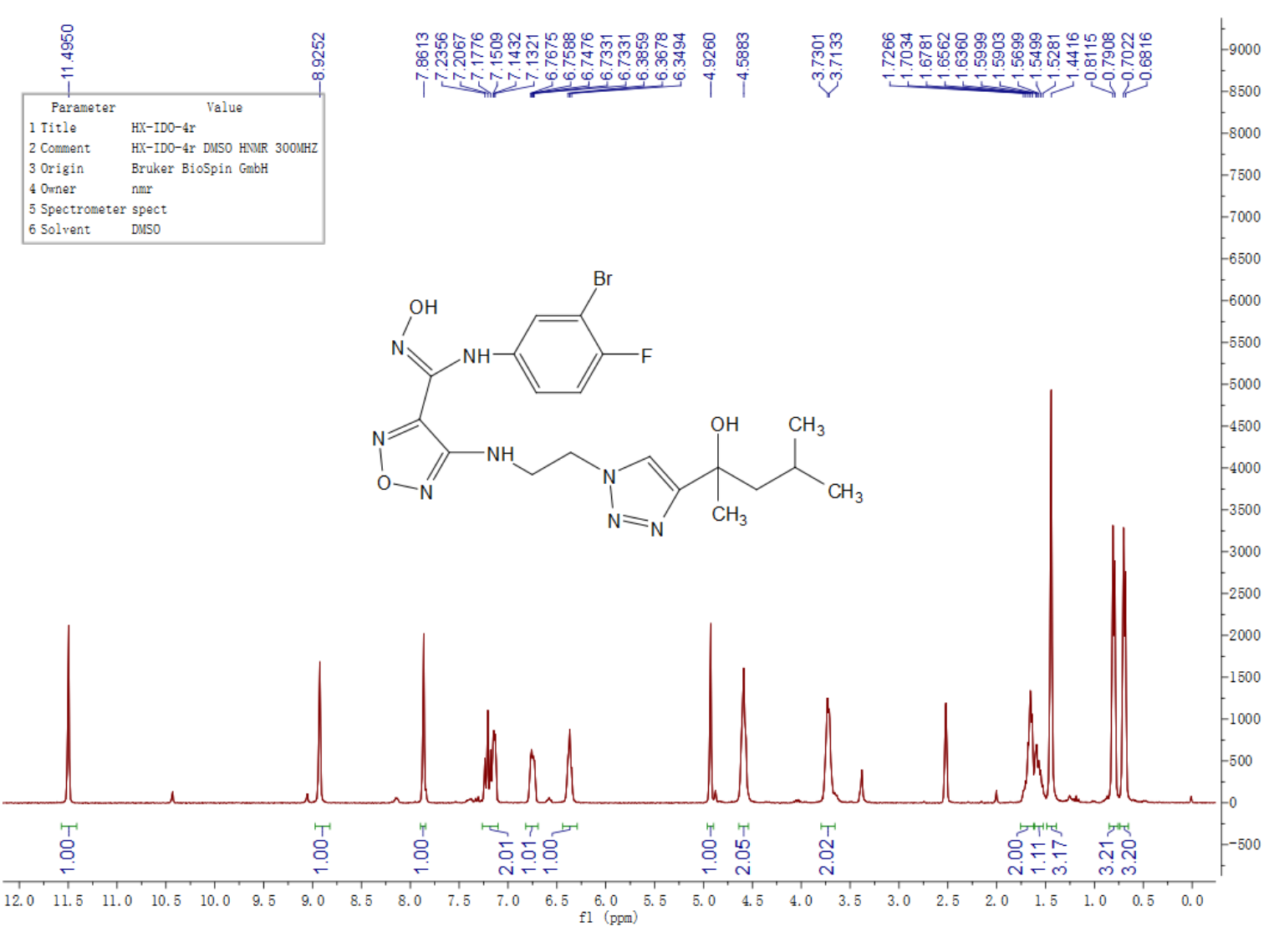

${ }^{13} \mathrm{C}$ NMR of $4 \mathbf{r}$

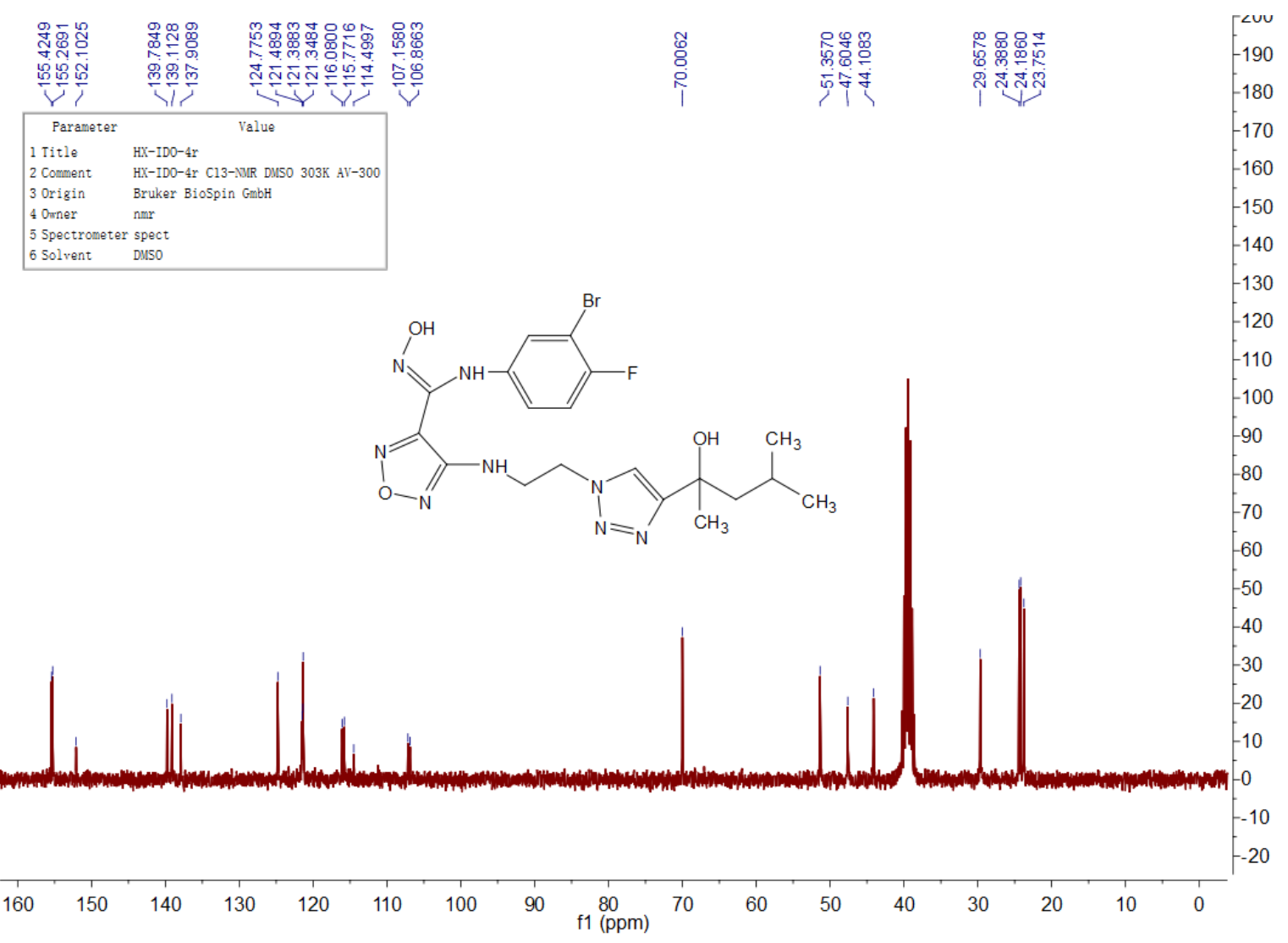


${ }^{1} \mathrm{H}$ NMR of $4 s$
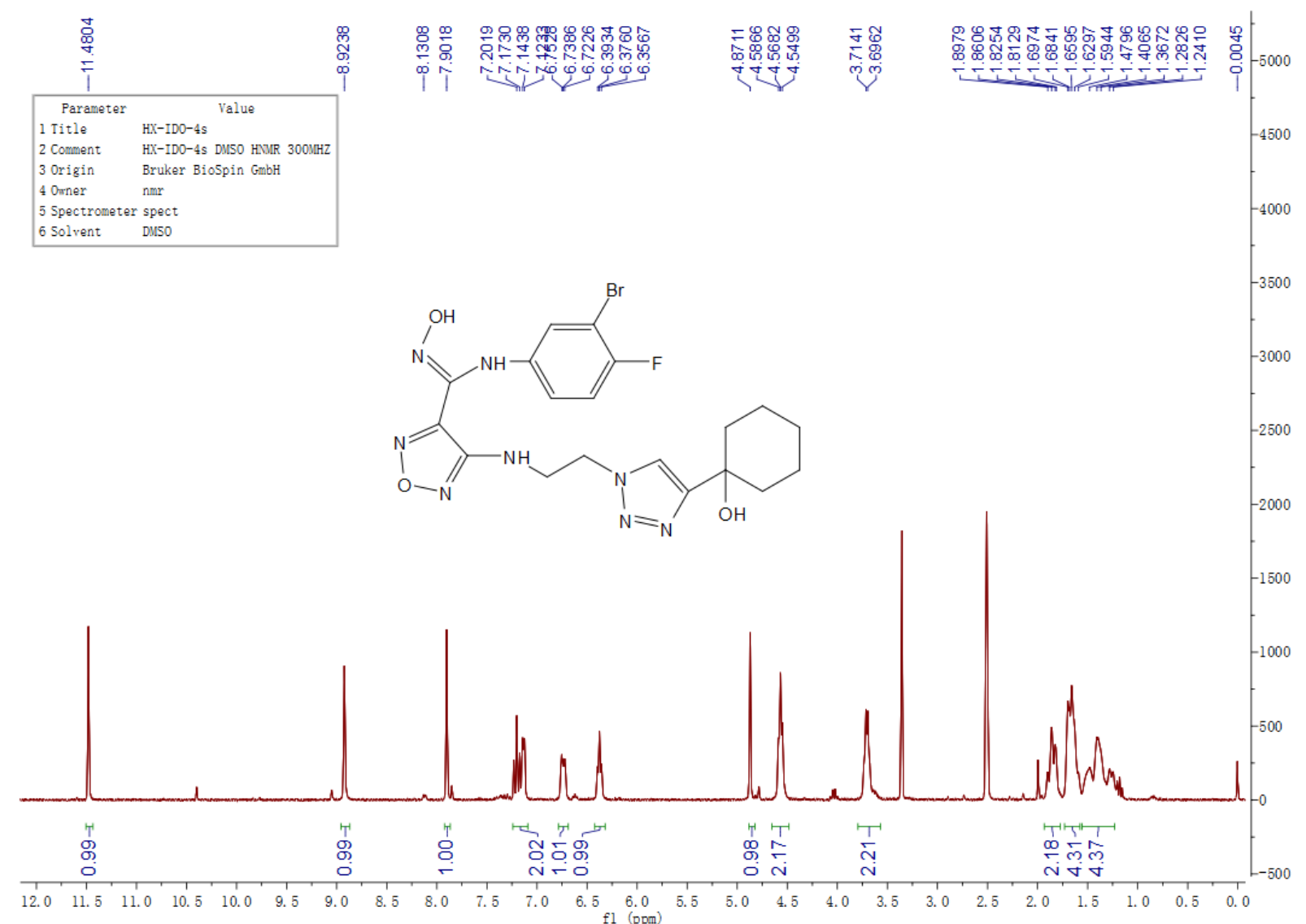

${ }^{13} \mathrm{C}$ NMR of $4 \mathrm{~s}$

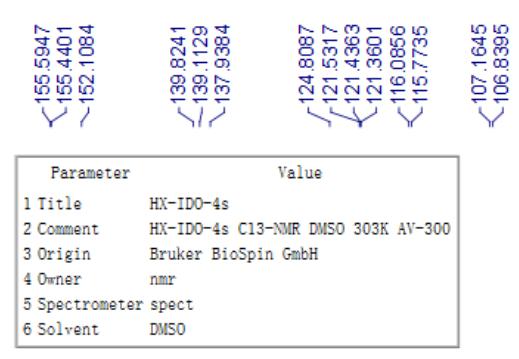

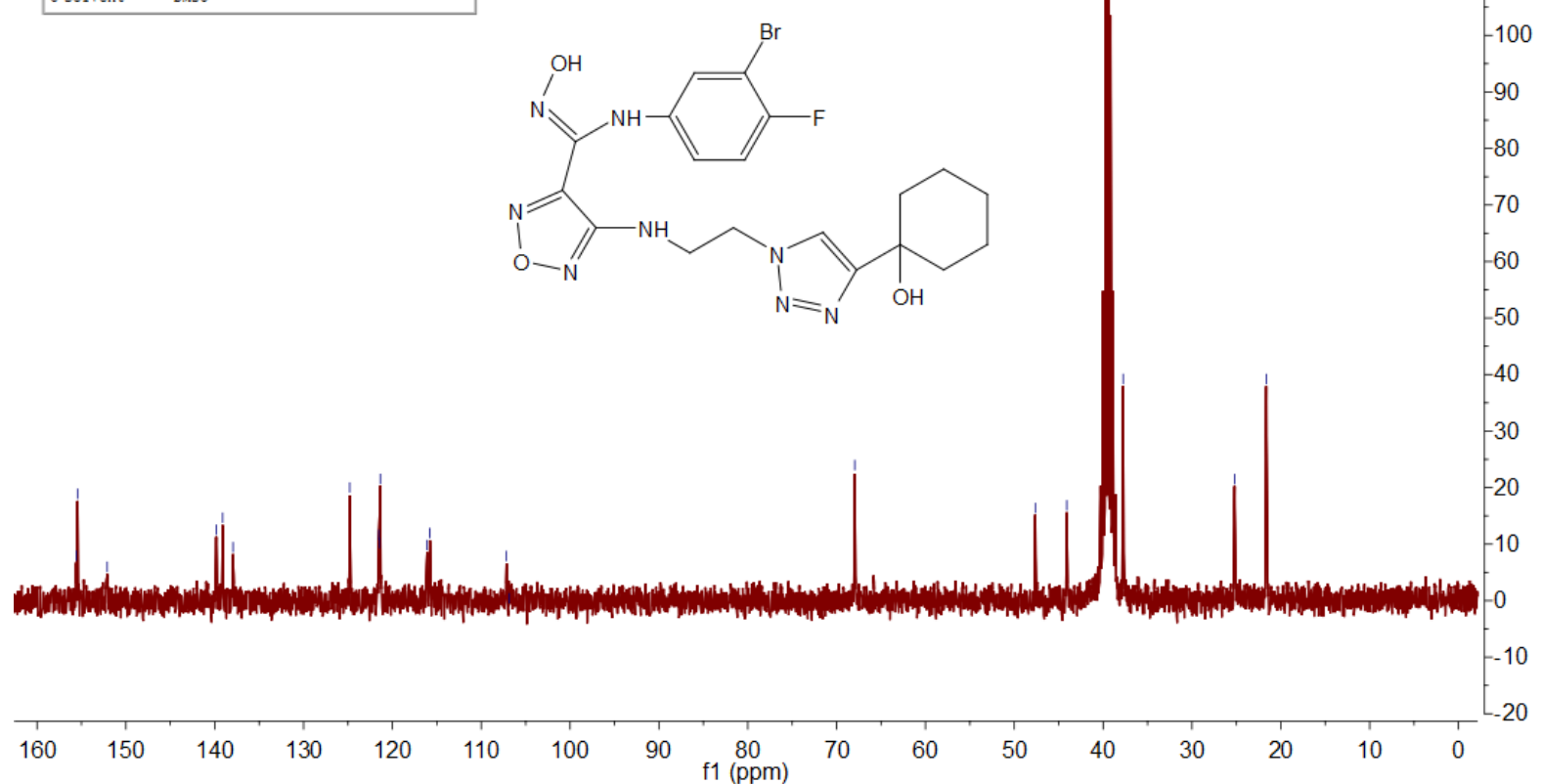

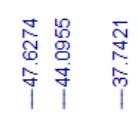

产

$5^{-100}$

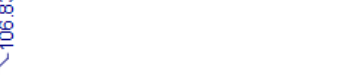


${ }^{1} \mathrm{H}$ NMR of $\mathbf{4 t}$

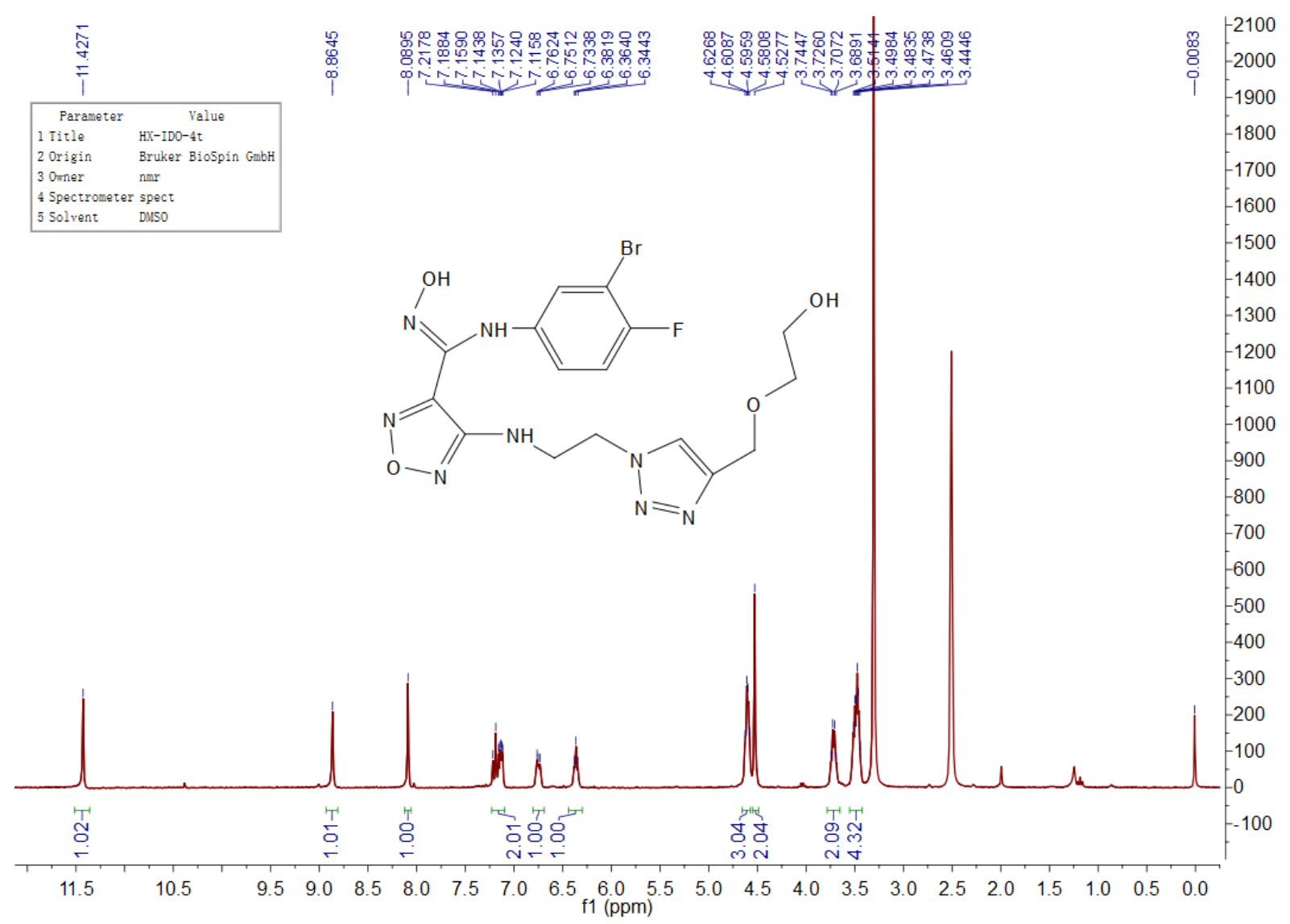

${ }^{13} \mathrm{C}$ NMR of $4 t$

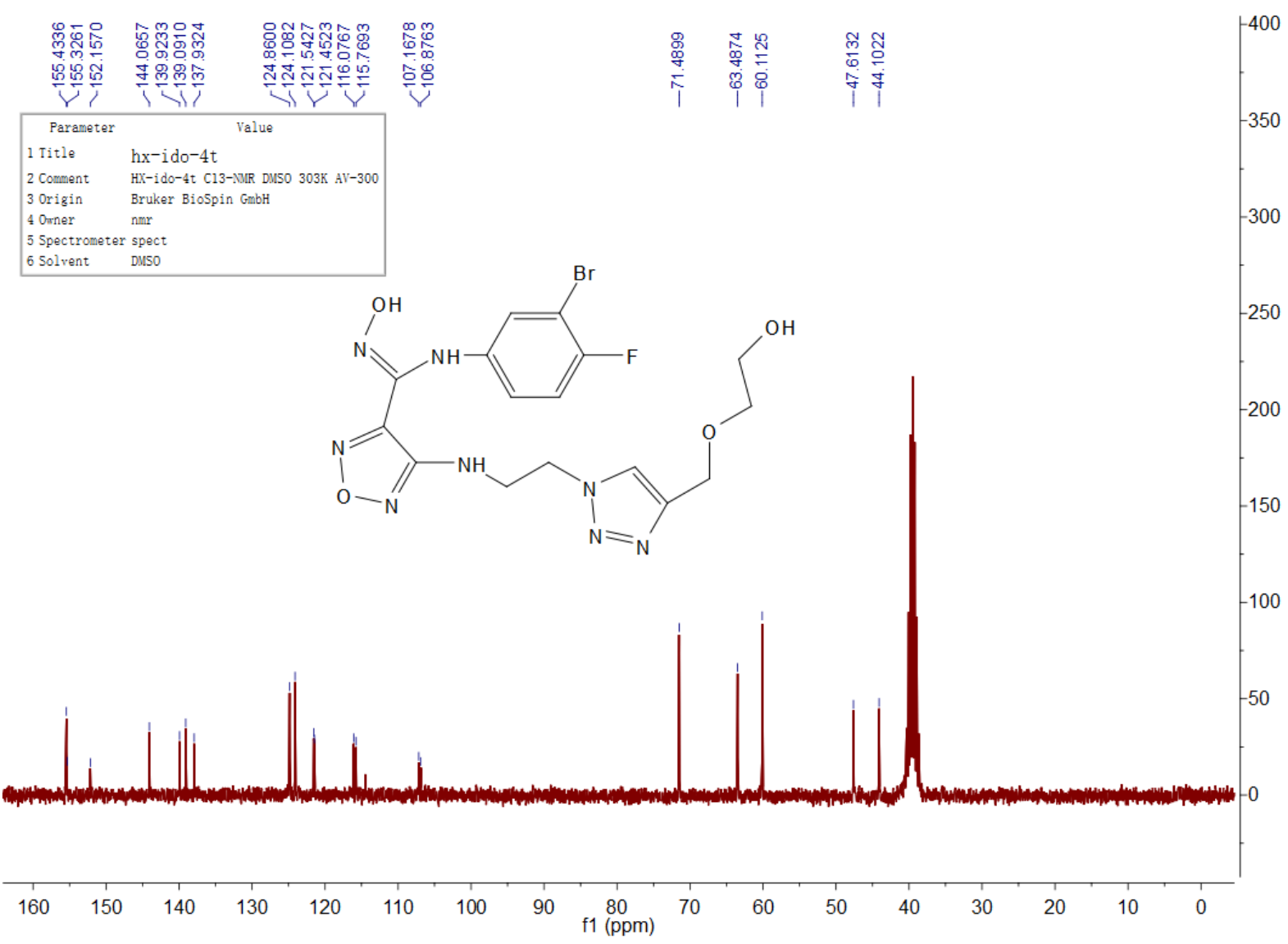


${ }^{1} \mathrm{H}$ NMR of $4 \mathbf{u}$

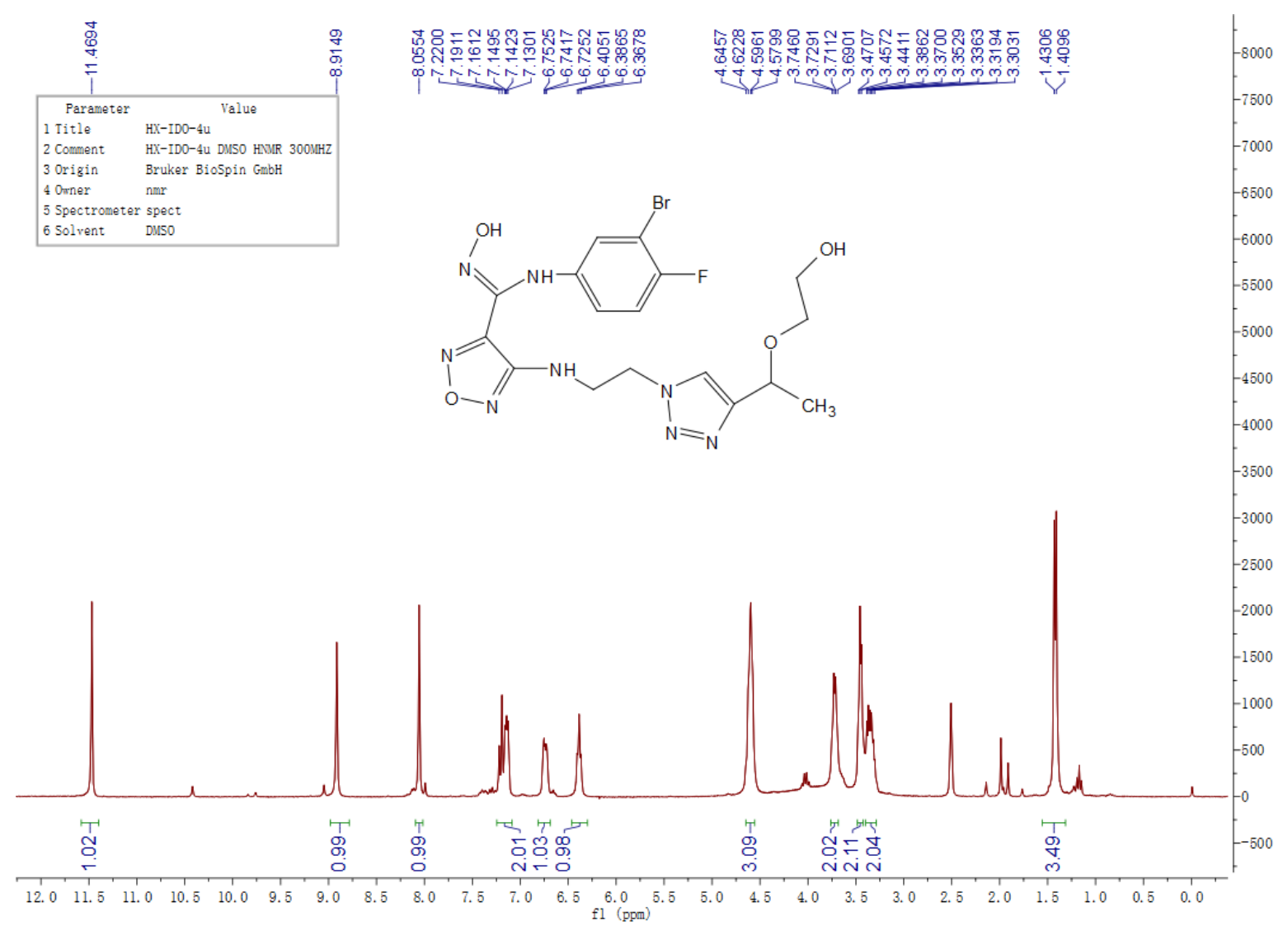

${ }^{13} \mathrm{C}$ NMR of $4 \mathbf{u}$

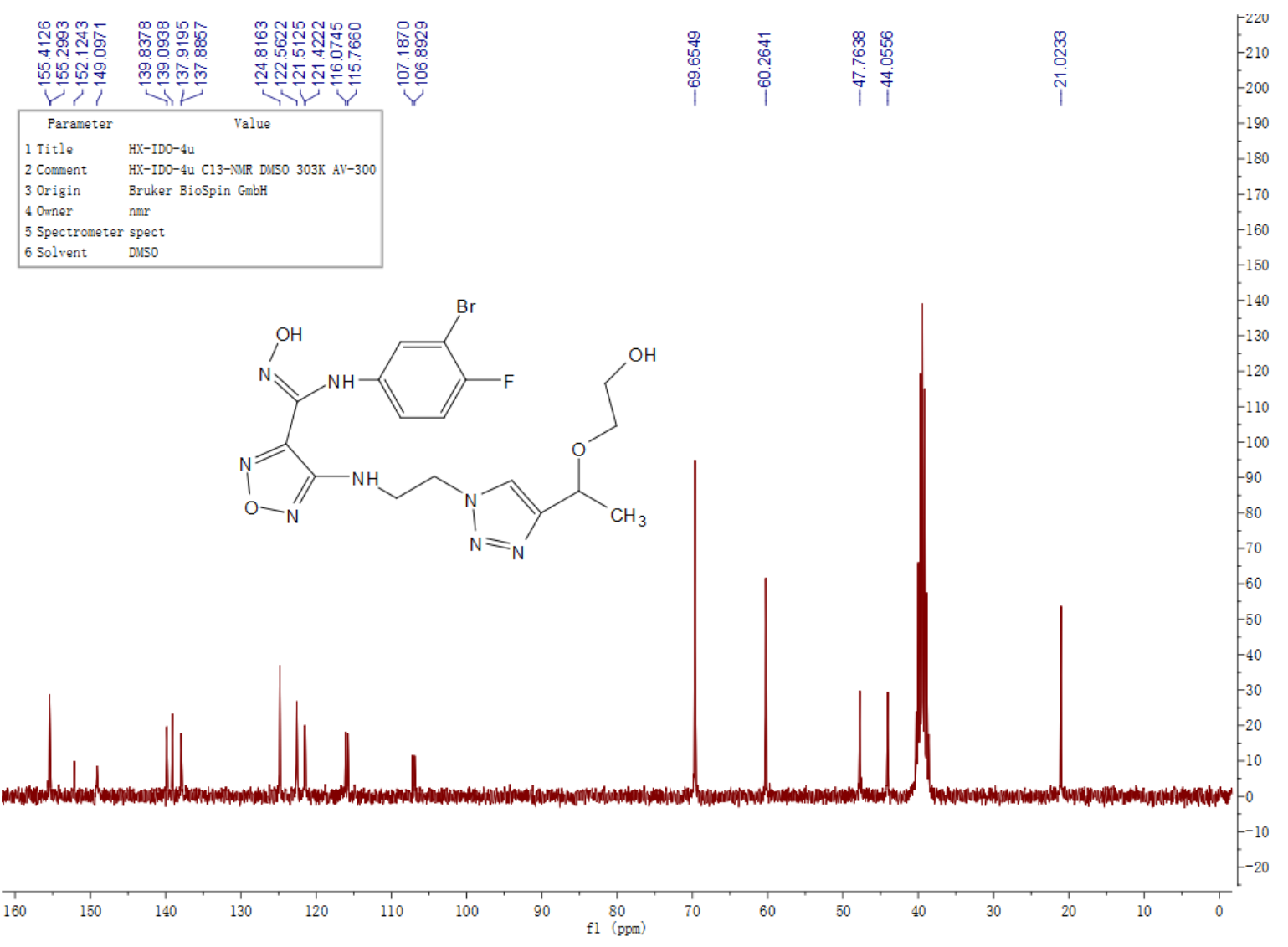


${ }^{1} \mathrm{H}$ NMR of $\mathbf{4 v}$

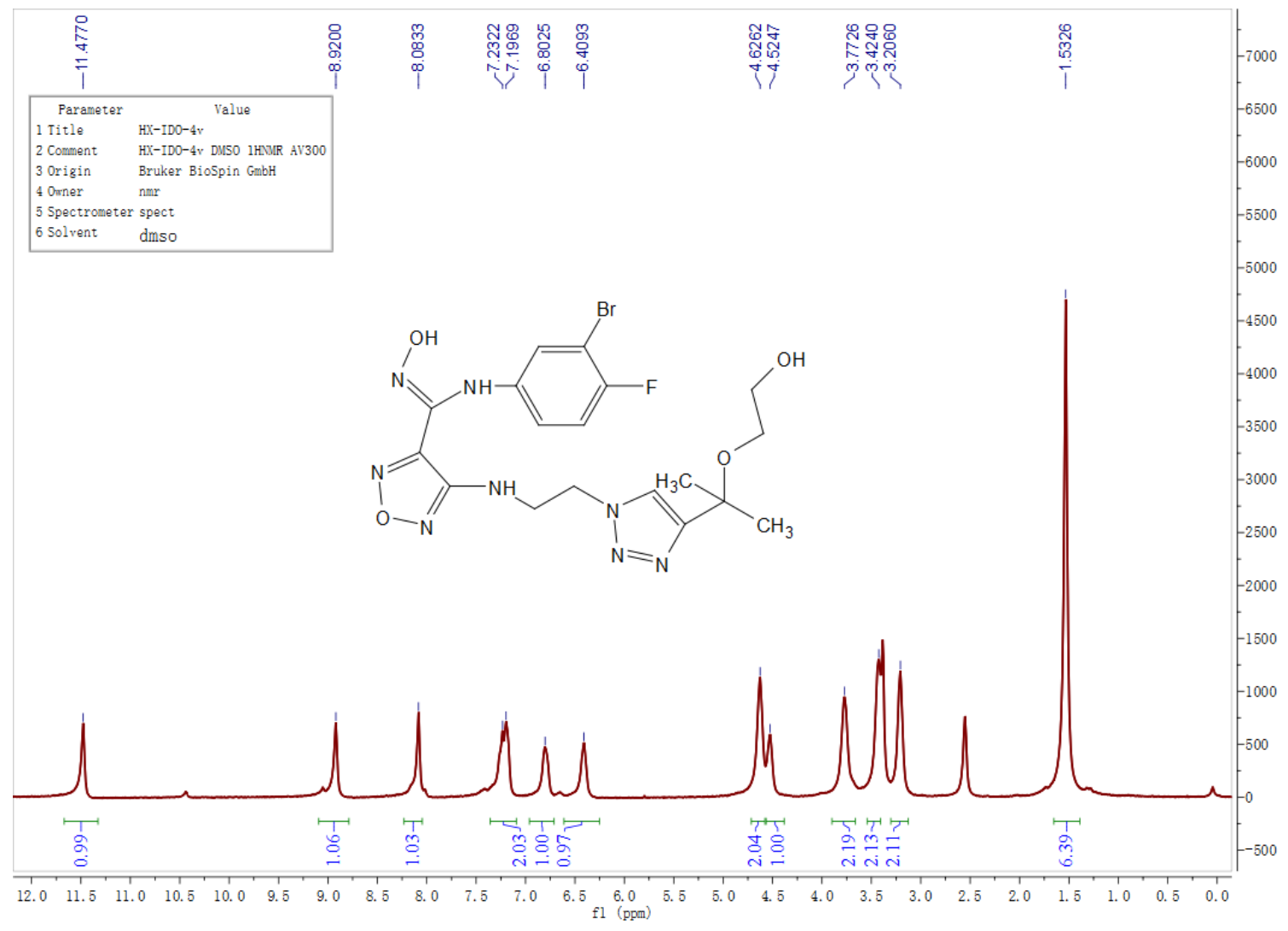

${ }^{13} \mathrm{C}$ NMR of $4 \mathbf{v}$

\begin{tabular}{|c|c|c|}
\hline 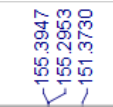 & 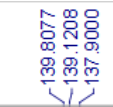 & 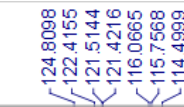 \\
\hline Parameter & & Value \\
\hline & \multirow{5}{*}{\multicolumn{2}{|c|}{$\begin{array}{l}\text { HX-IDO-4v } \\
\text { HX-IDO-4v C13-NIR DMSO 303K AV-300 } \\
\text { Bruker Biospin GmbH } \\
\text { nmm } \\
\text { r spect } \\
\text { nveso }\end{array}$}} \\
\hline $\begin{array}{l}2 \text { comment } \\
3 \text { Origin }\end{array}$ & & \\
\hline 4 Orner & & \\
\hline 5 Spectromet & & \\
\hline 6 Solv & & \\
\hline
\end{tabular}
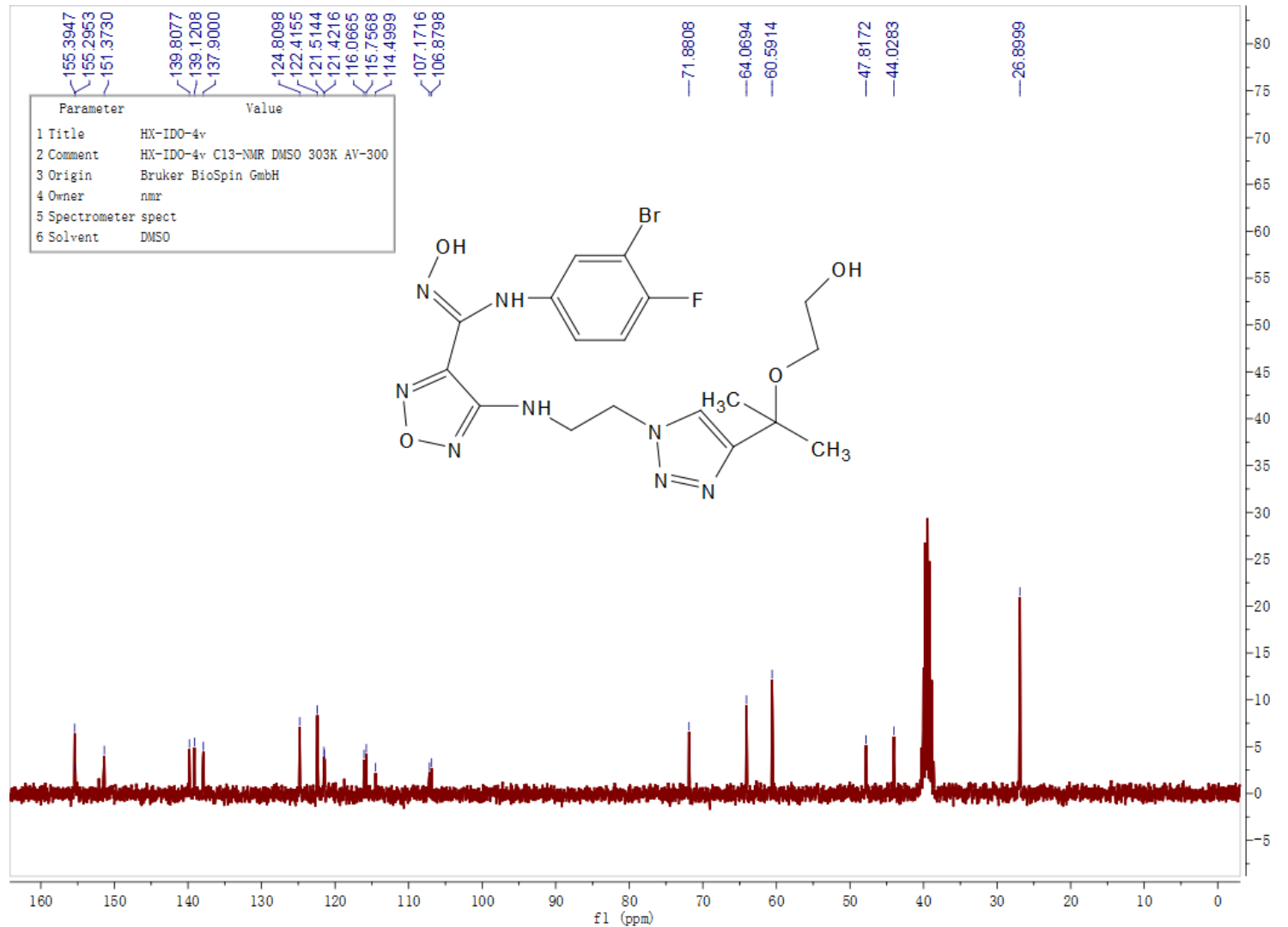

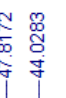

$\mathscr{8}$
$\stackrel{8}{8}$
$\stackrel{0}{0}$
$\stackrel{0}{1}$ 


\section{HRMS Spectra of All Target Compounds}

\section{HRMS of $4 a$}

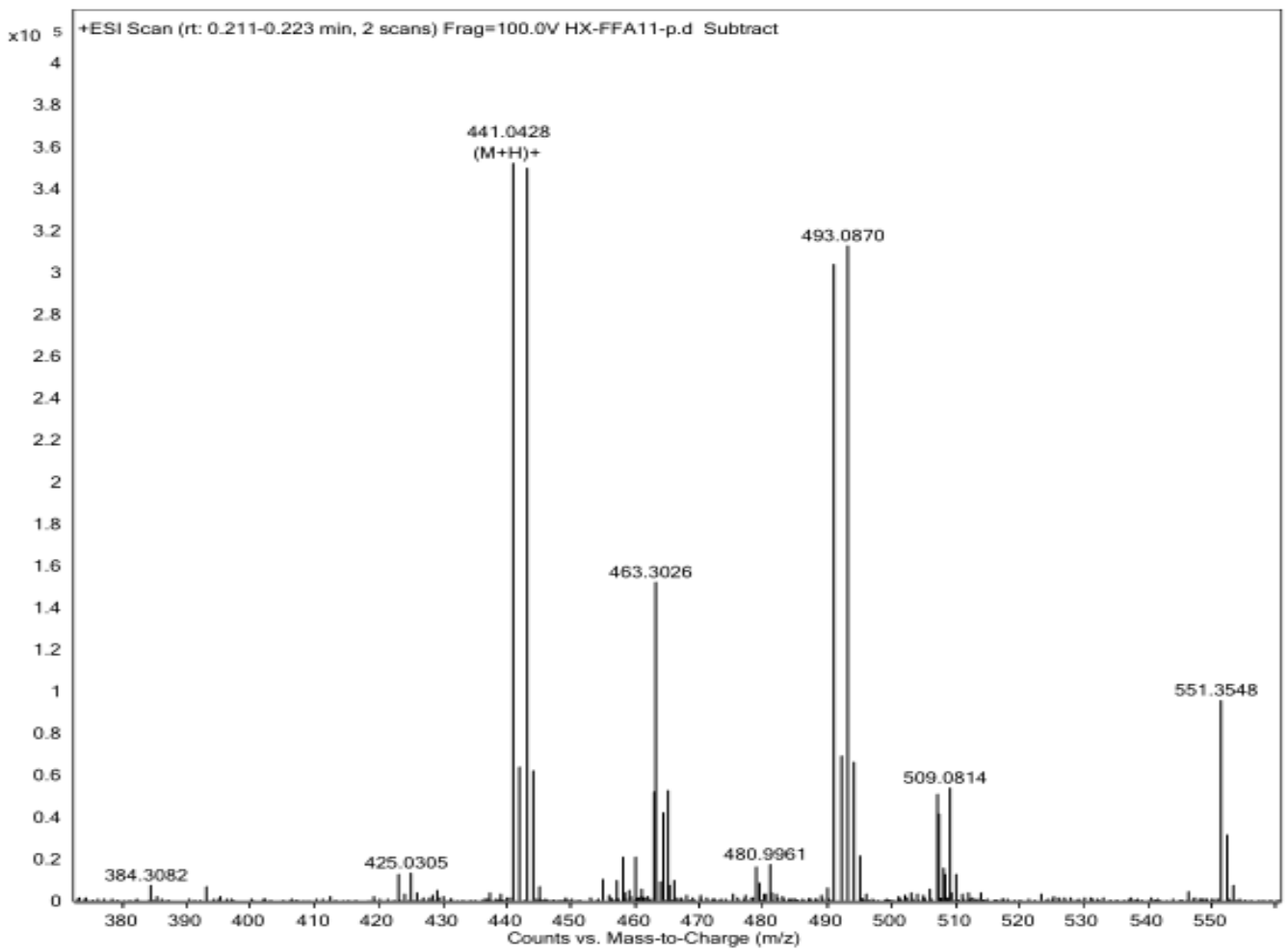

HRMS of $\mathbf{4 b}$

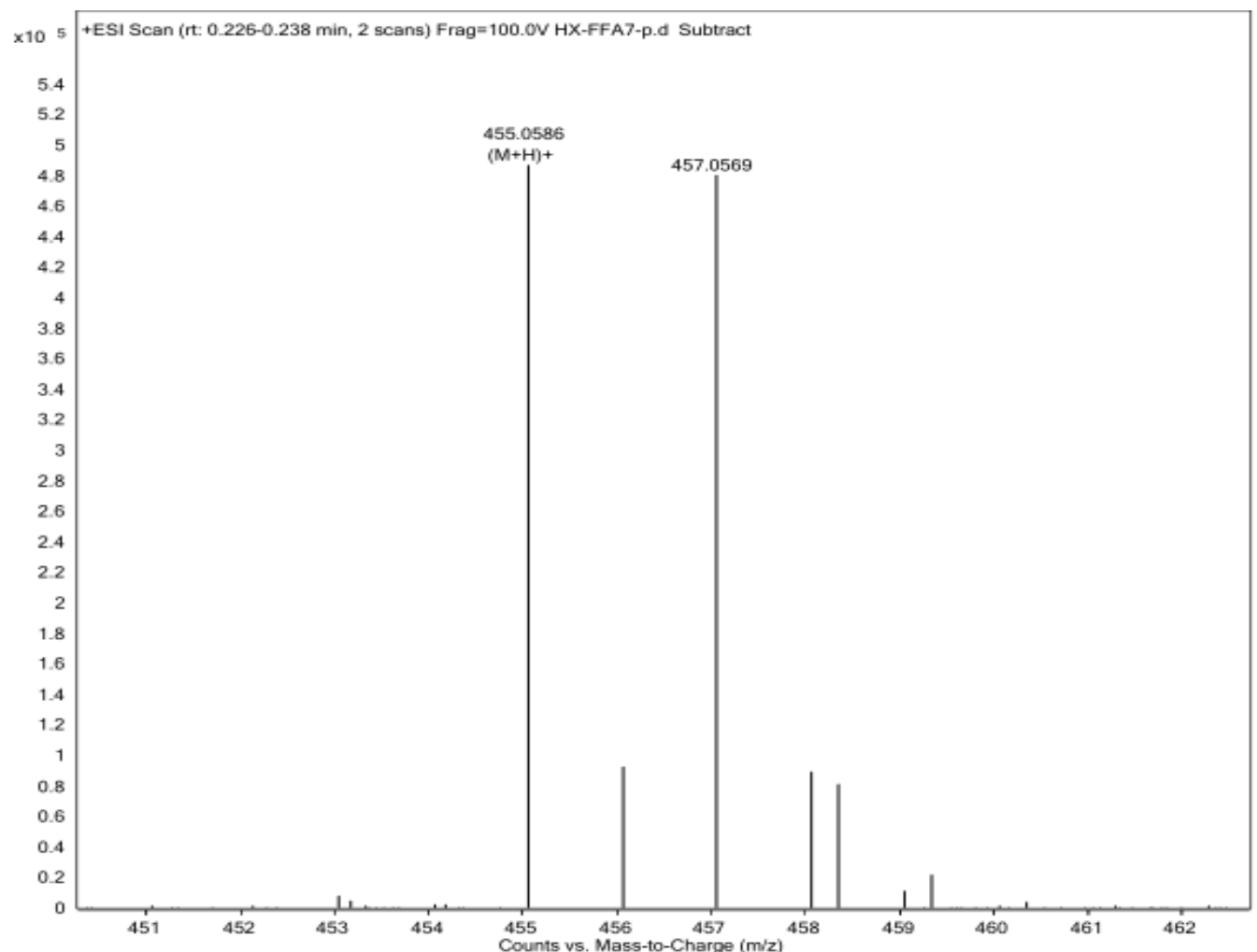




\section{HRMS of $4 \mathbf{c}$}

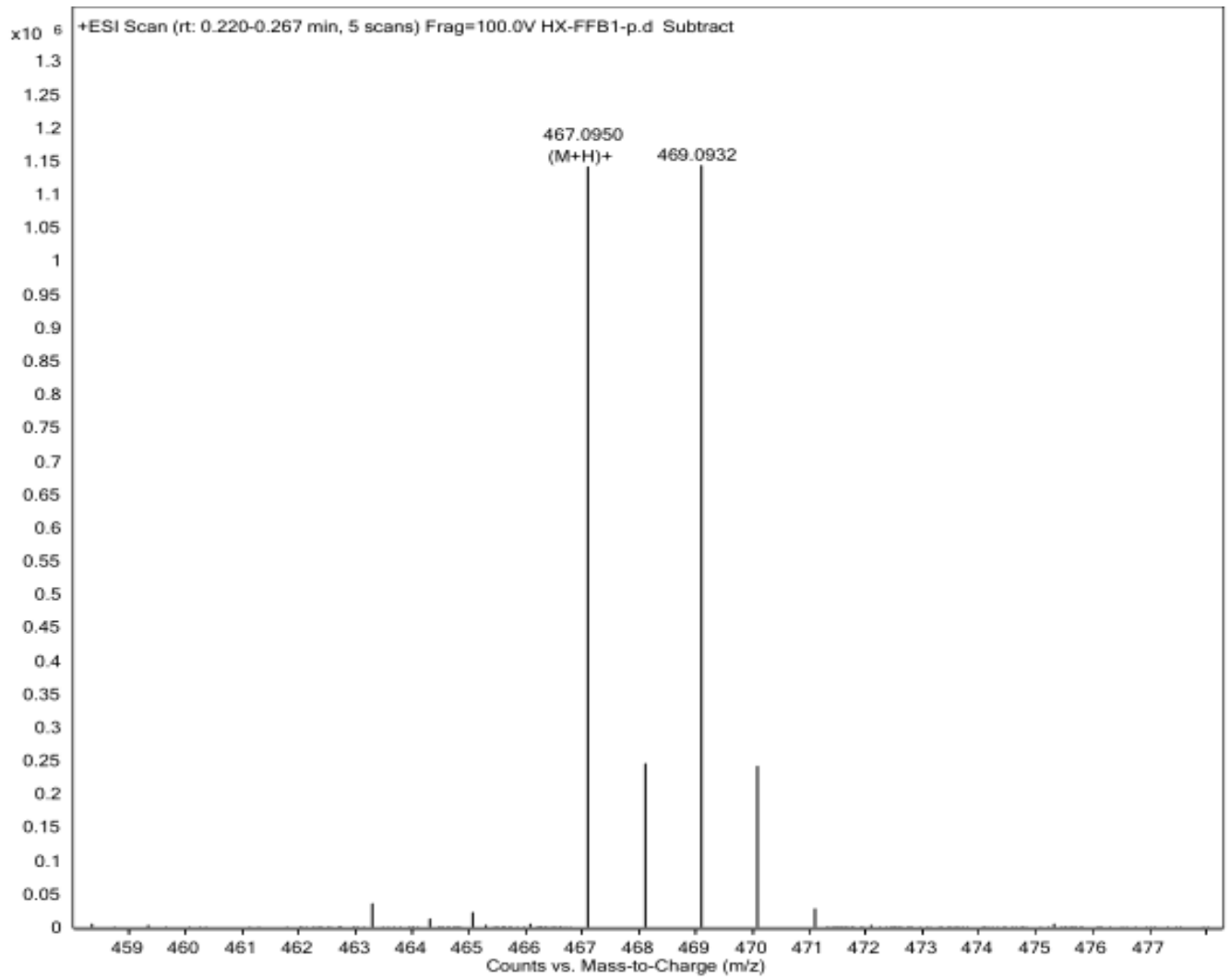

\section{HRMS of 4d}

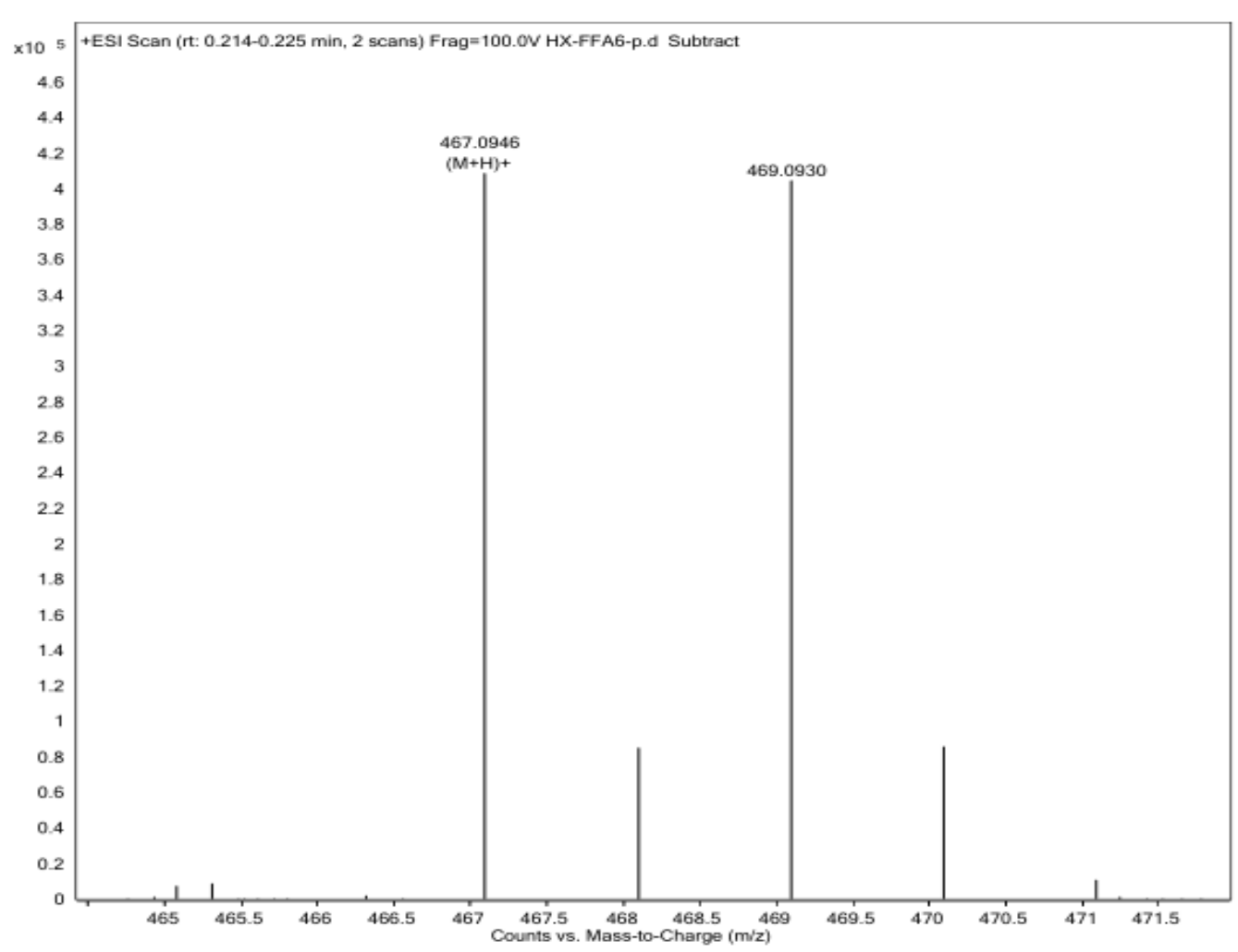




\section{HRMS of $4 e$}

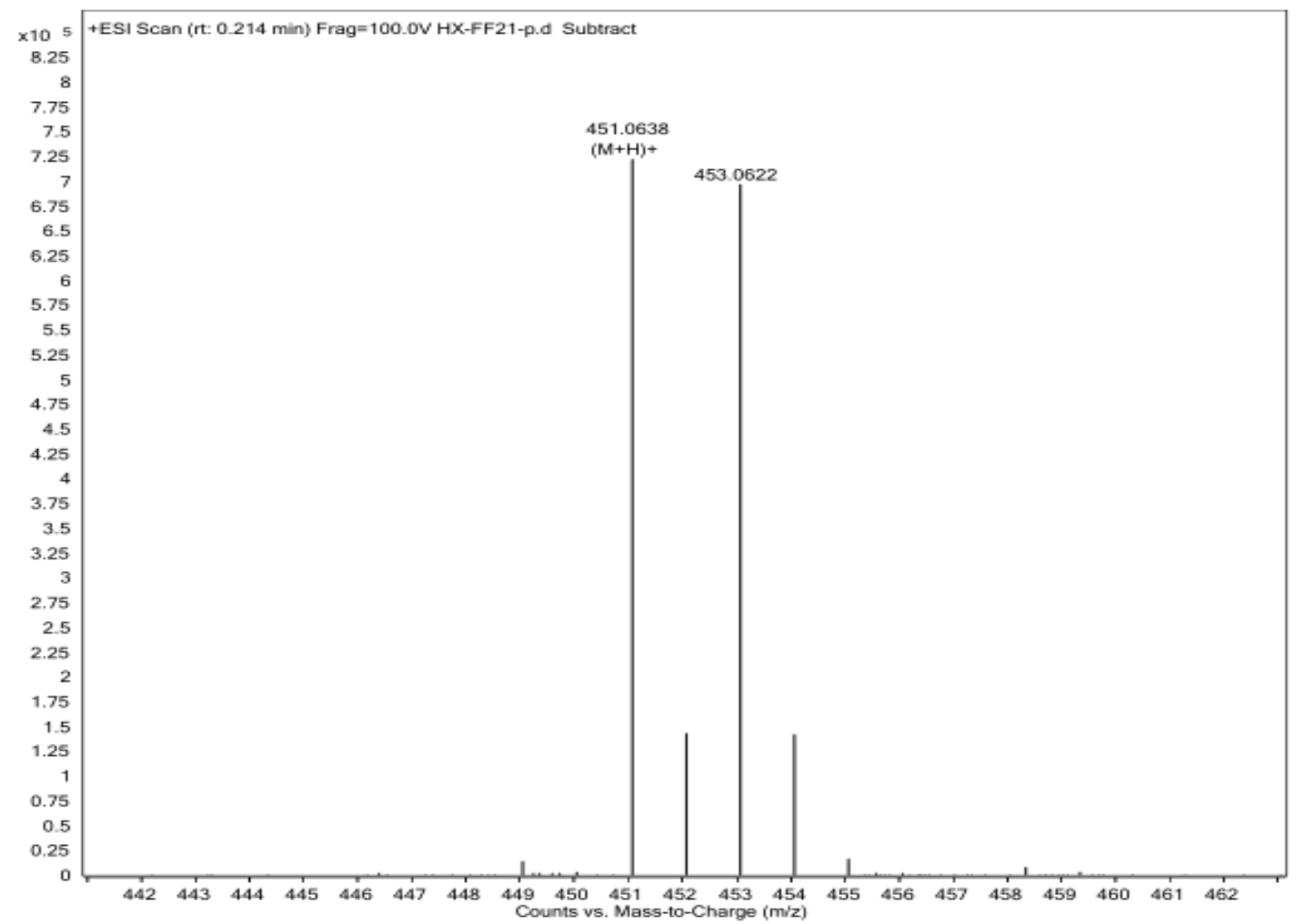

HRMS of $\mathbf{4 f}$

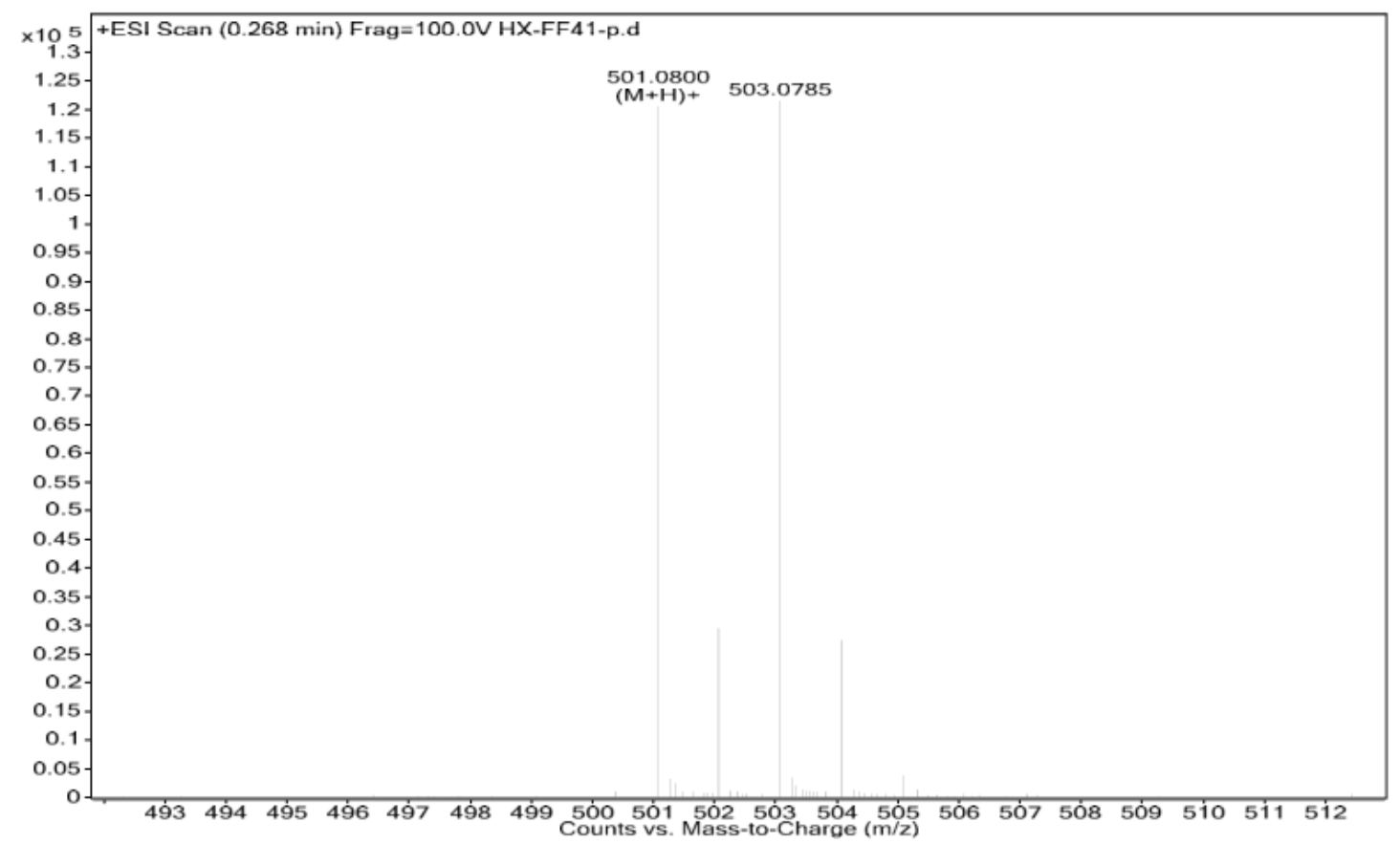




\section{HRMS of $\mathbf{4 g}$}

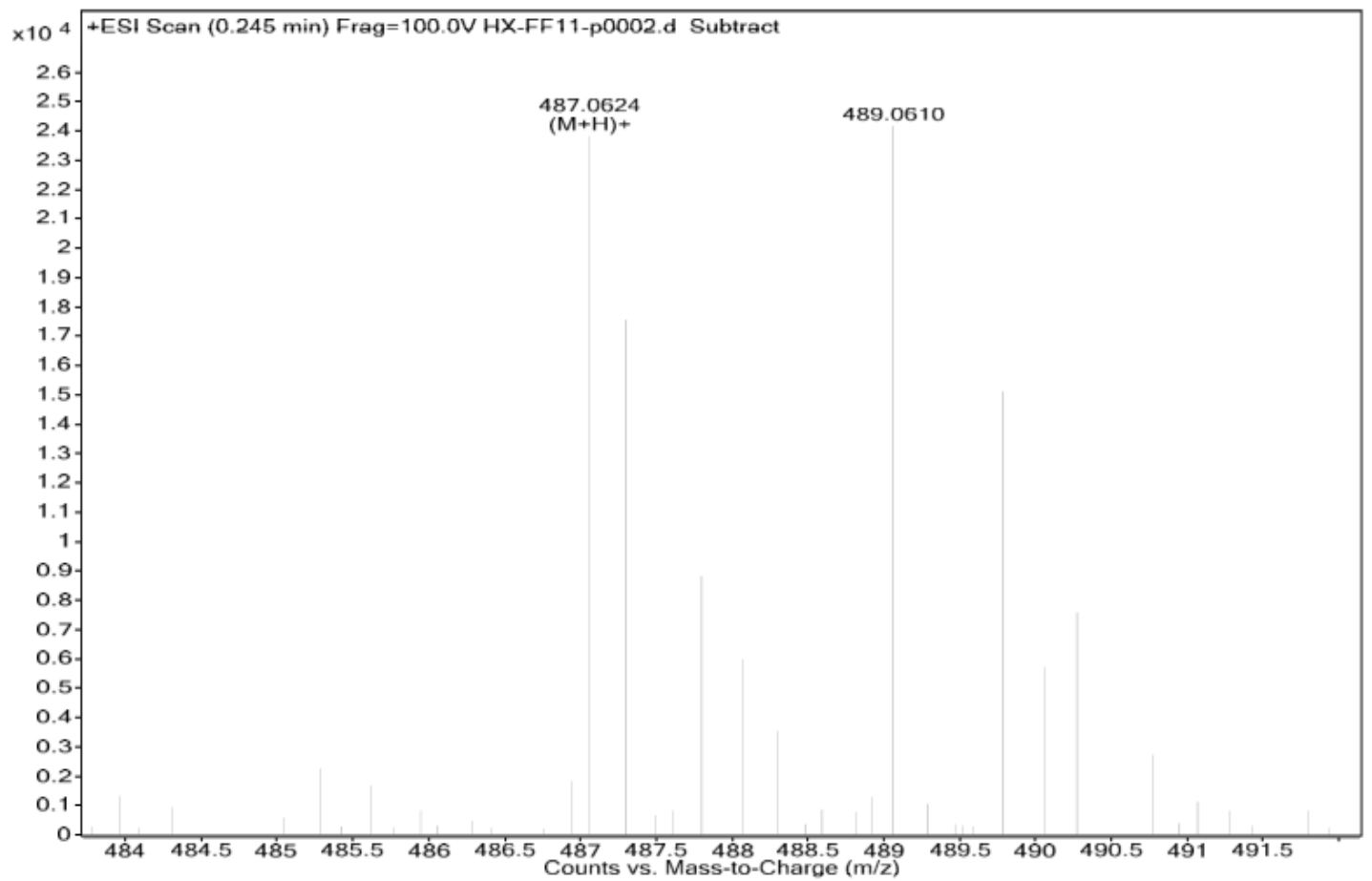

HRMS of $\mathbf{4 h}$

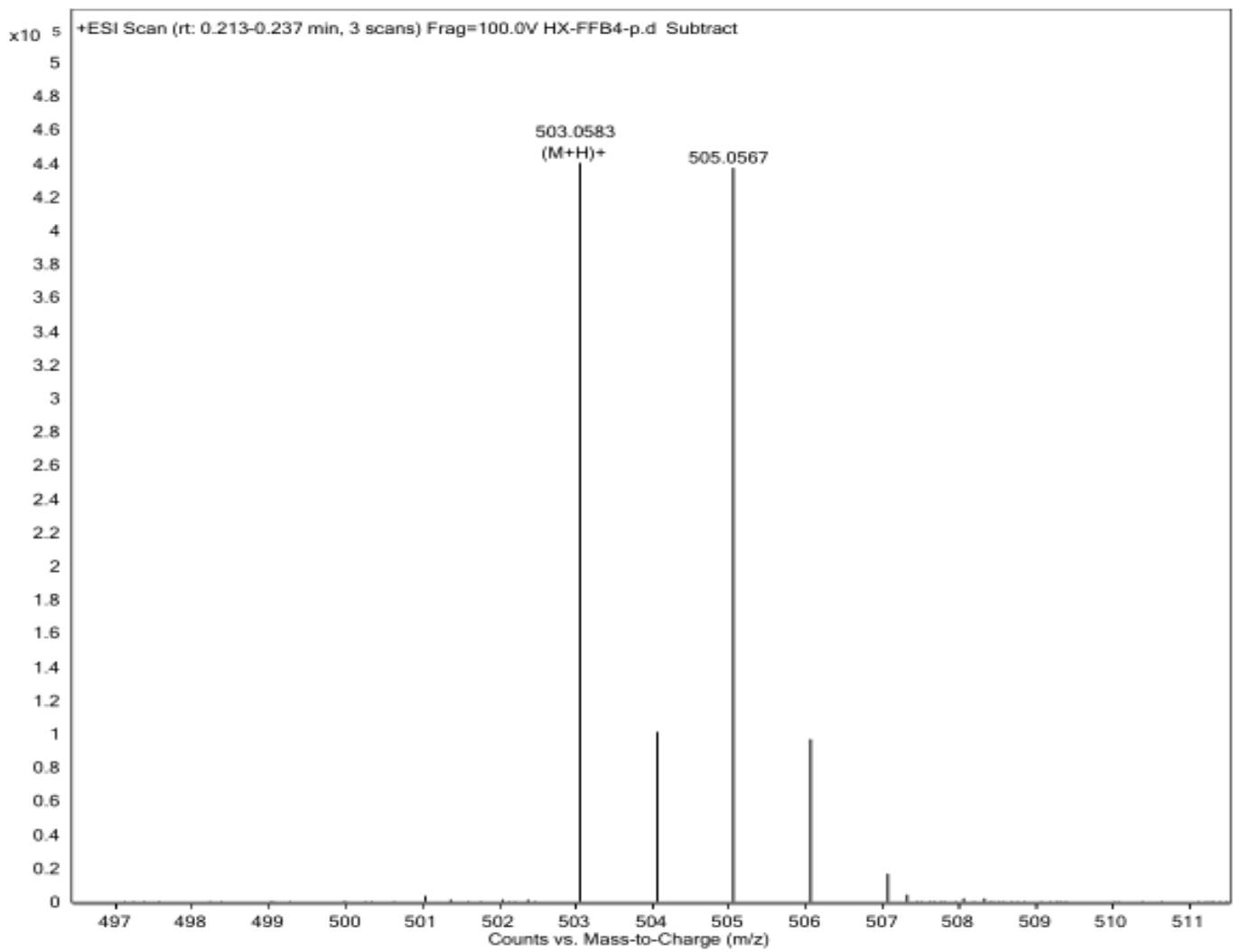


HRMS of $\mathbf{4 i}$

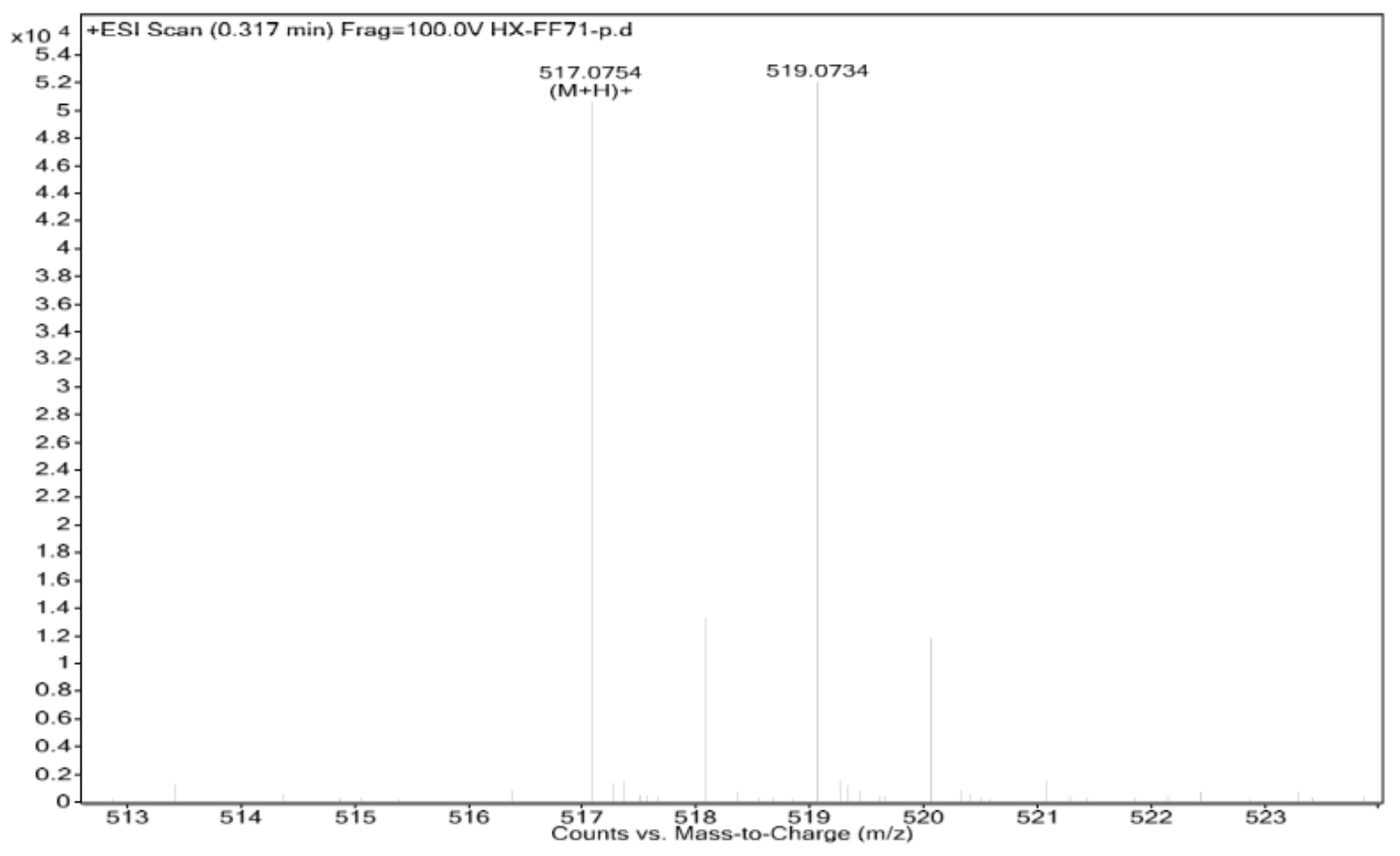

HRMS of $\mathbf{4 j}$

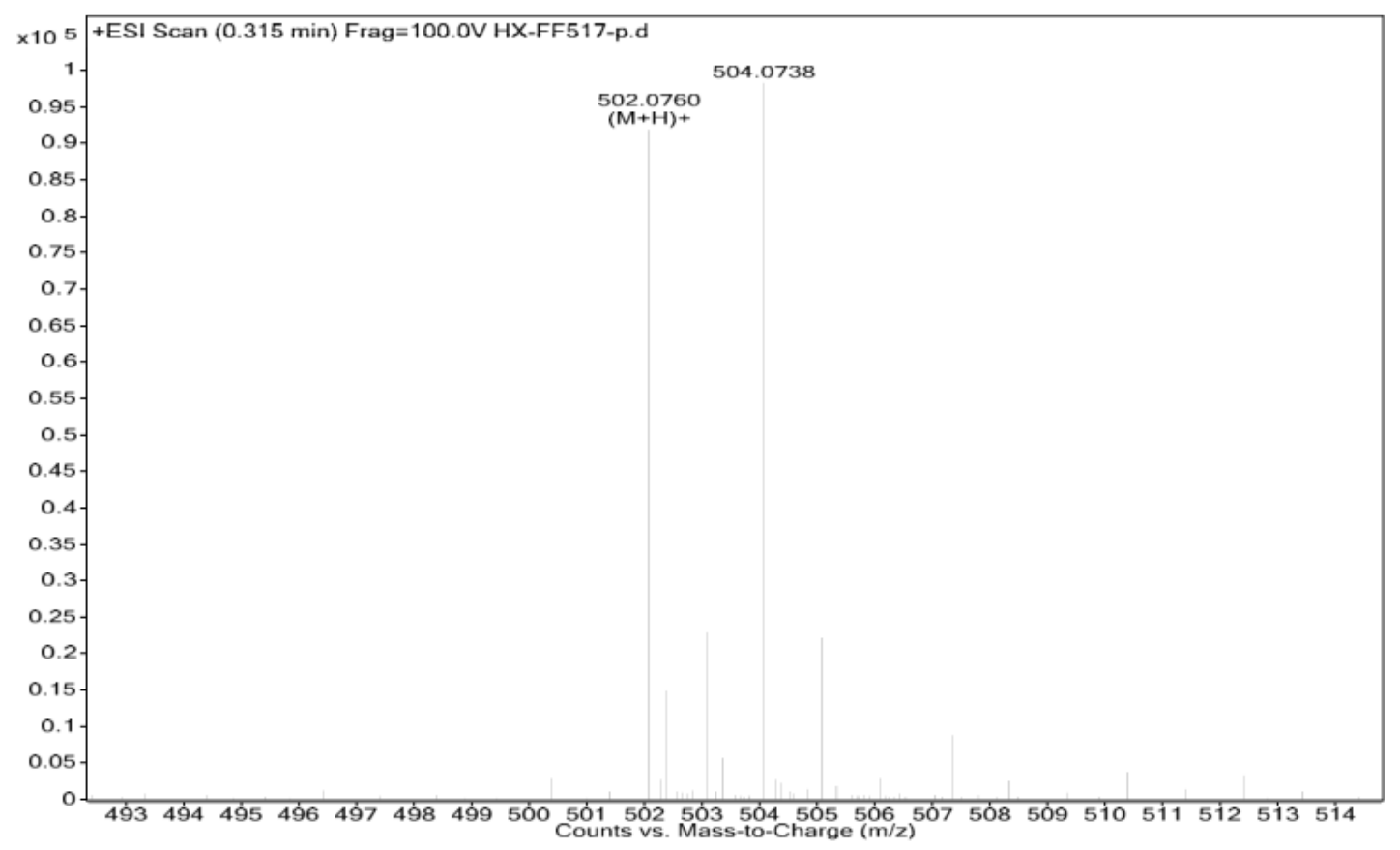


HRMS of $\mathbf{4 k}$

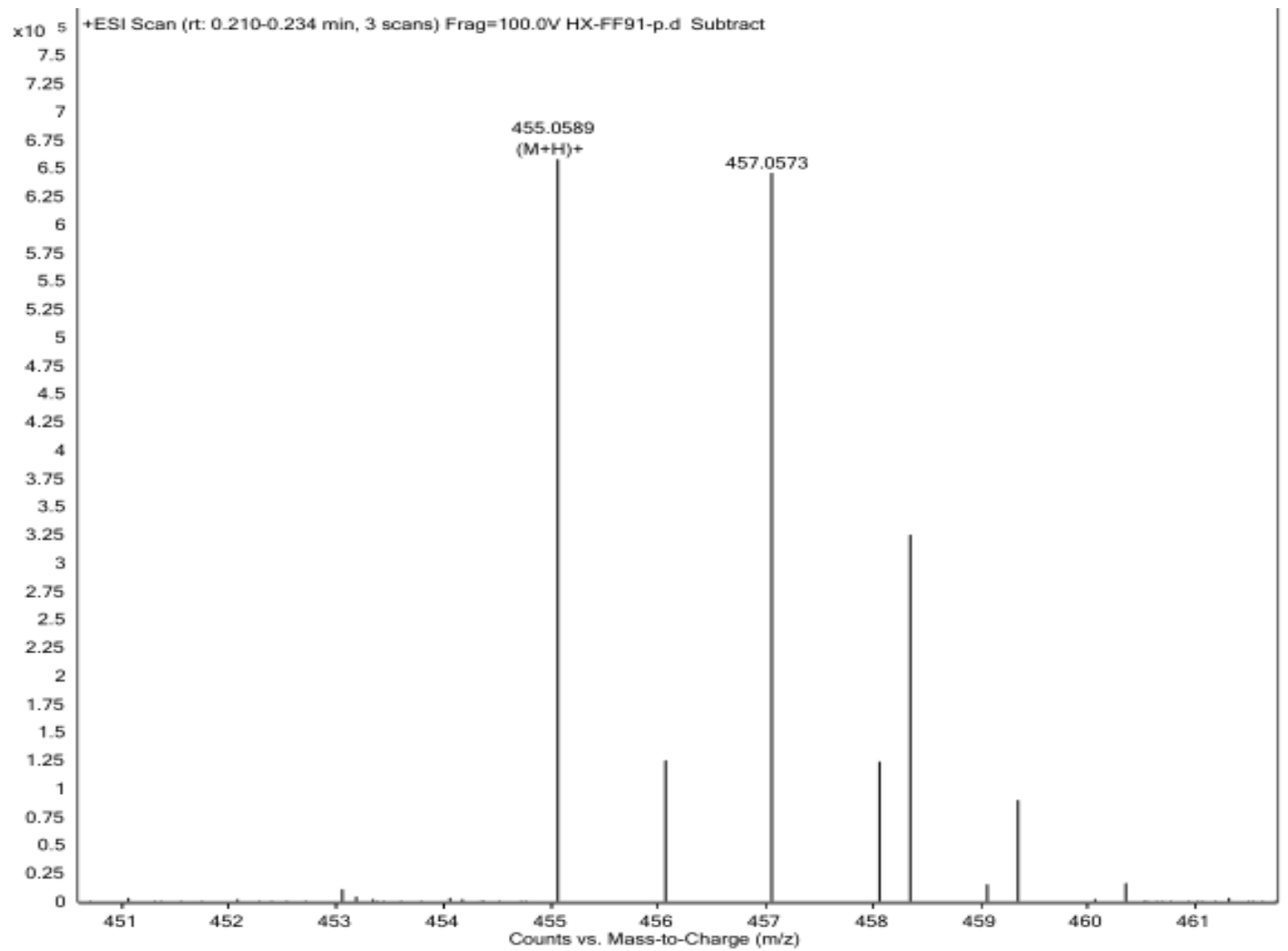

\section{HRMS of 4}

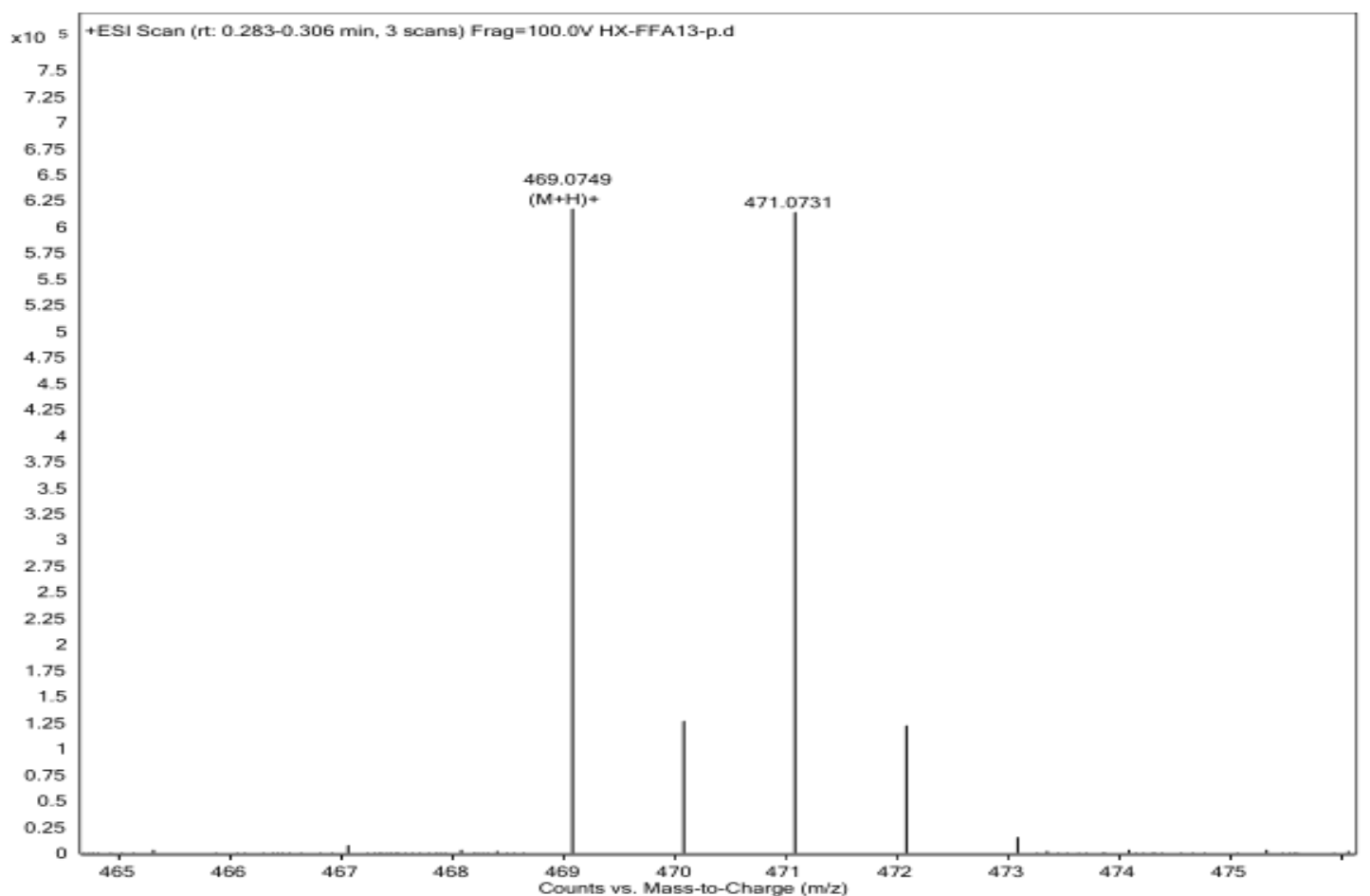




\section{HRMS of $4 \mathbf{m}$}

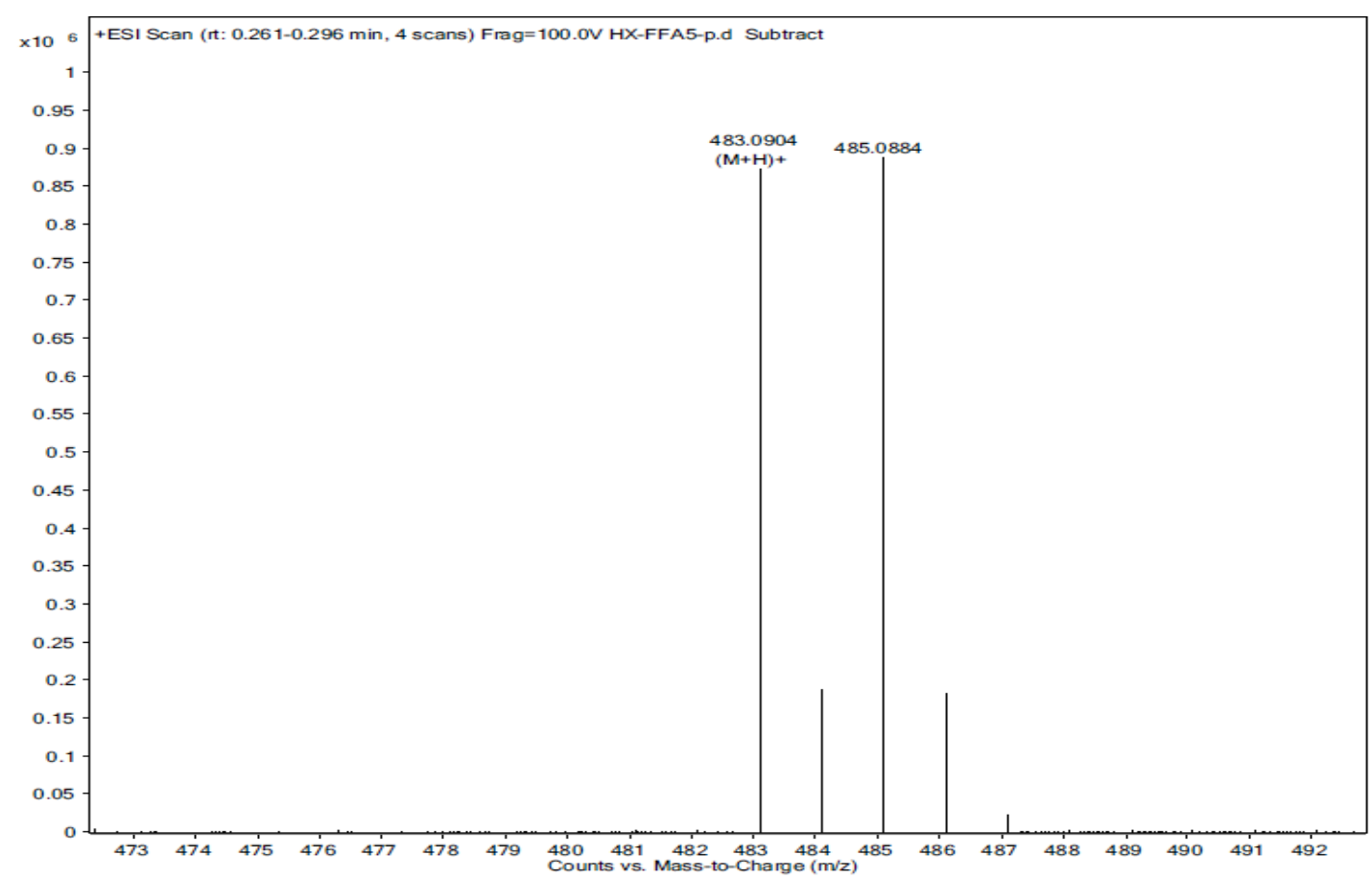

\section{HRMS of 4n}

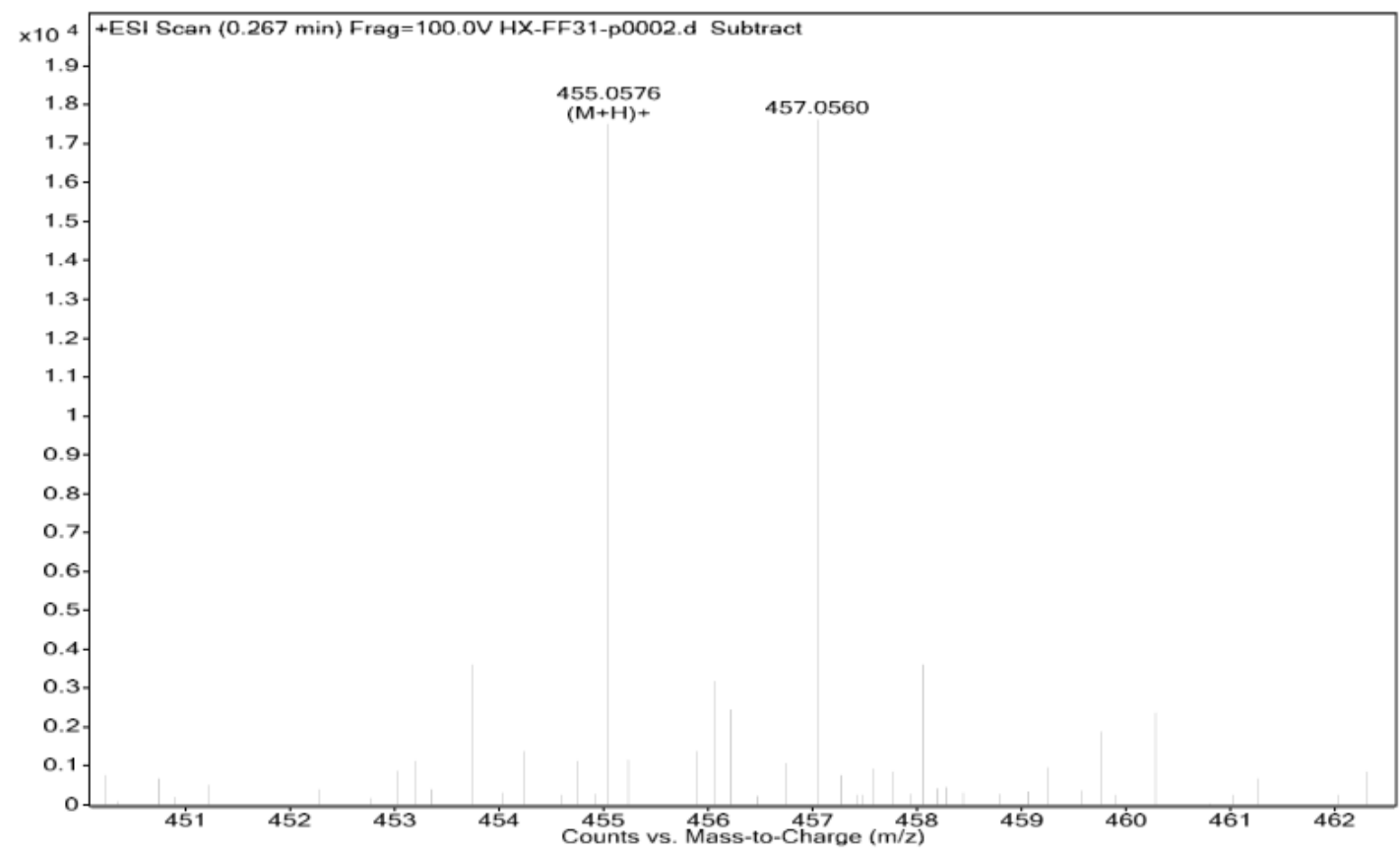




\section{HRMS of 40}

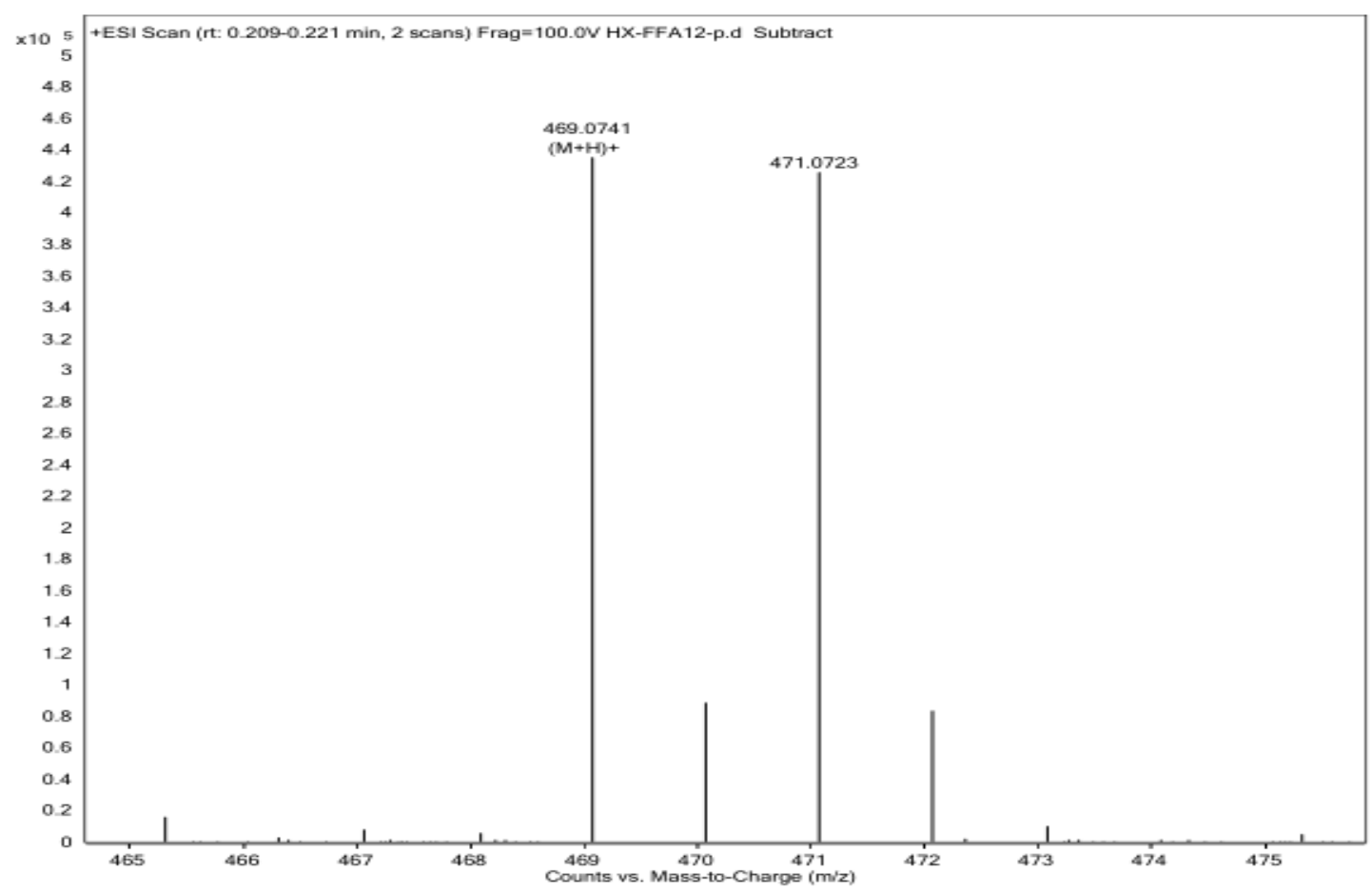

\section{HRMS of $4 p$}

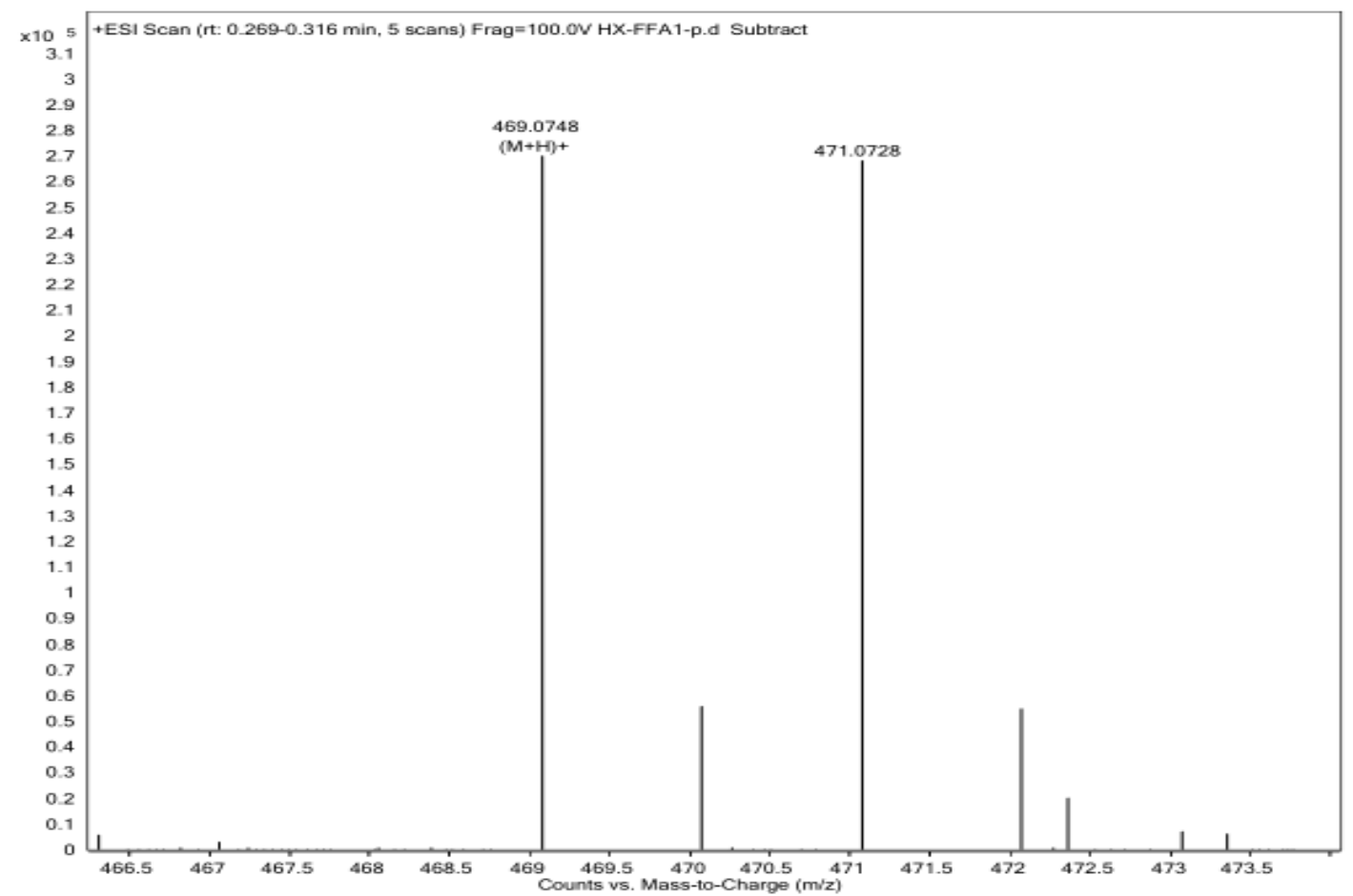




\section{HRMS of $\mathbf{4 q}$}

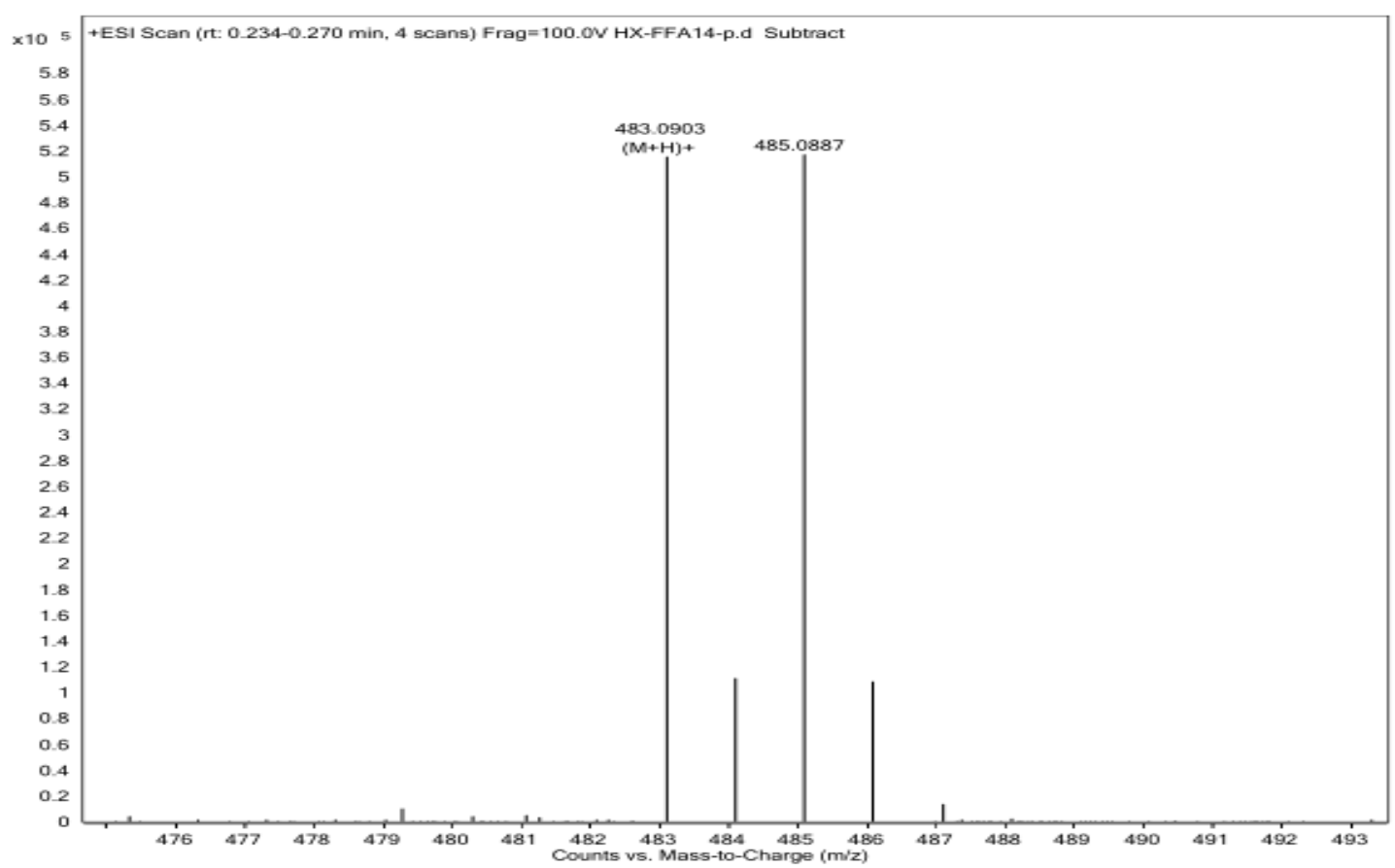

HRMS of 4r

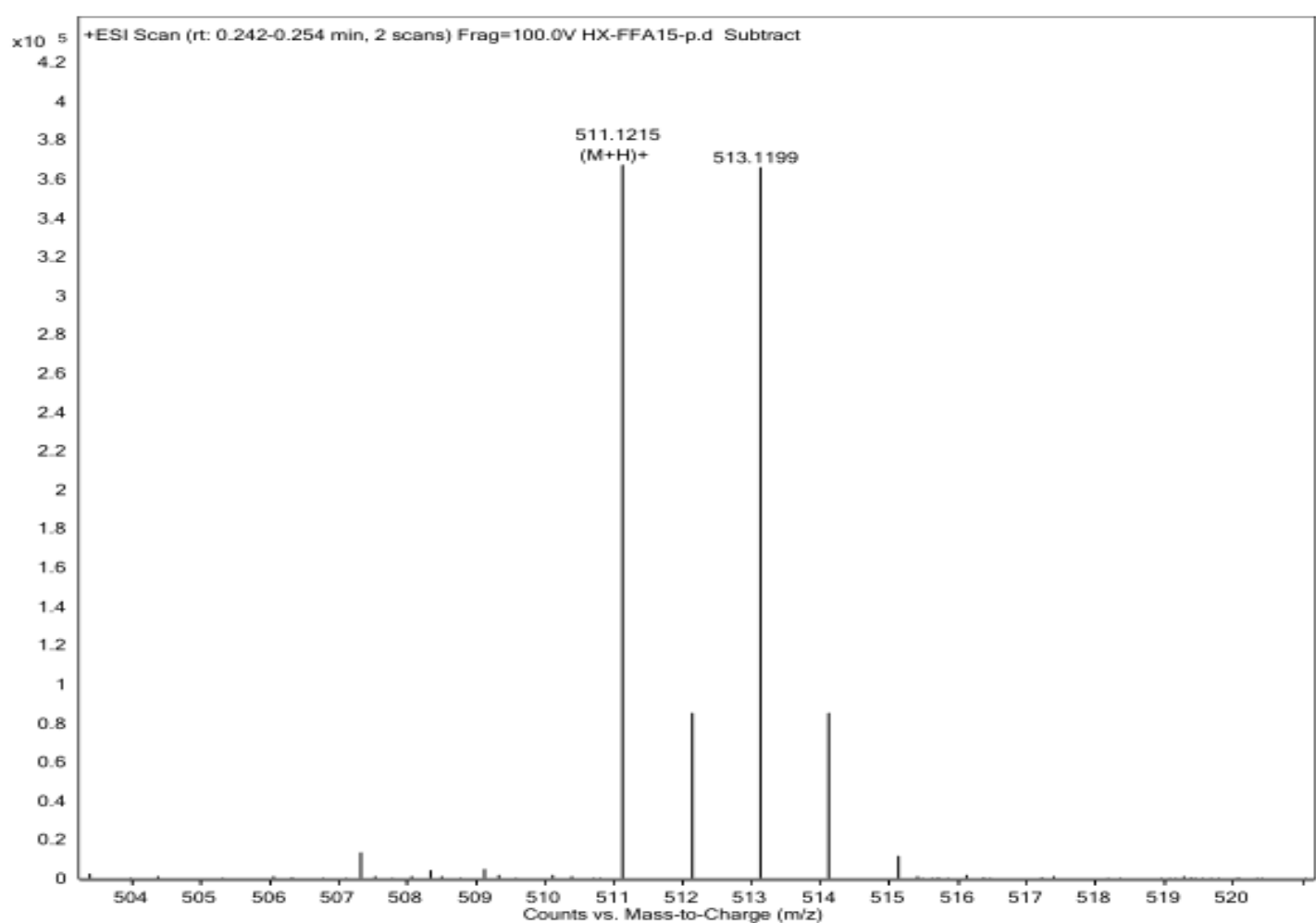




\section{HRMS of $4 \mathrm{~s}$}

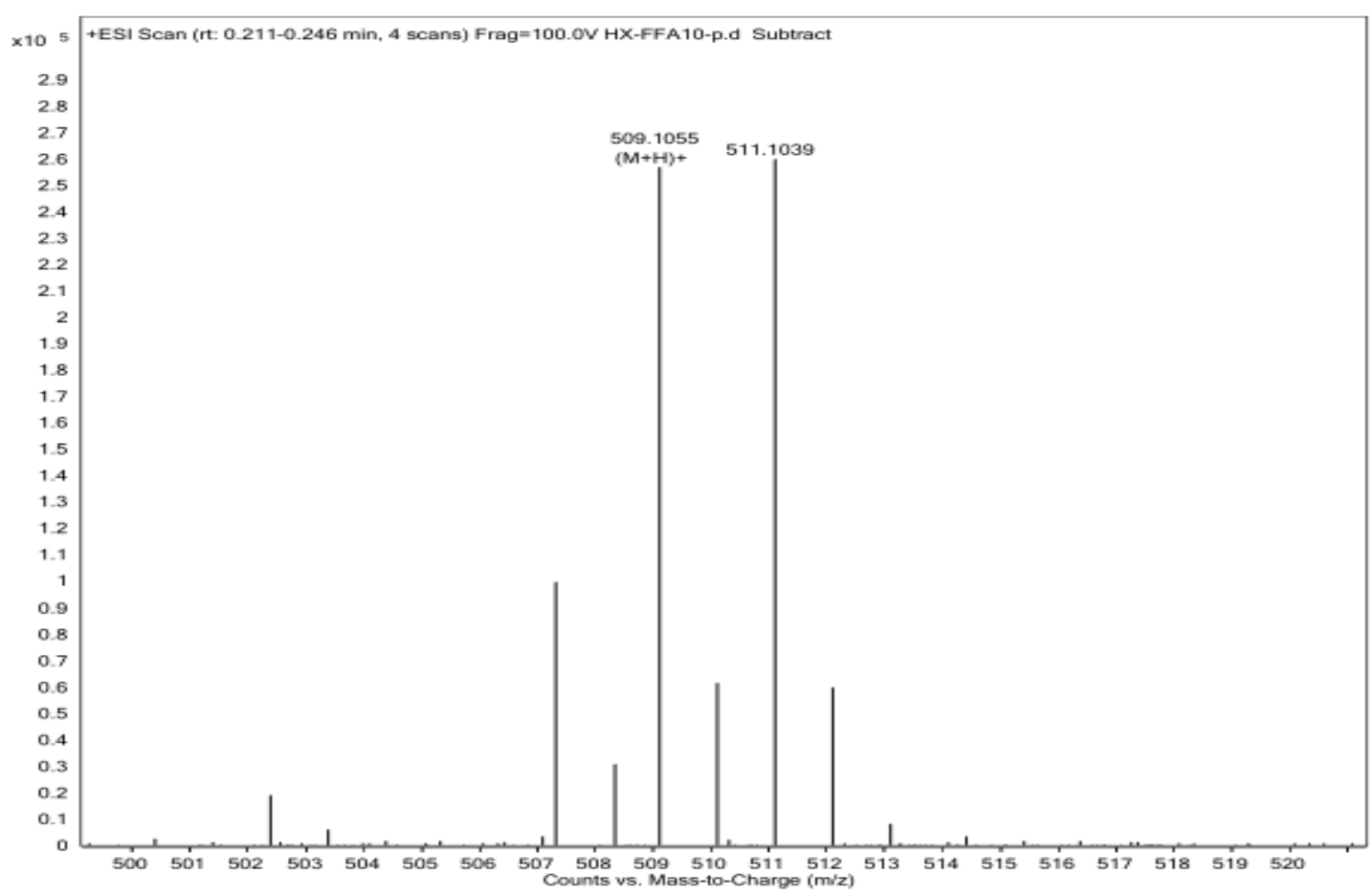

\section{HRMS of $\mathbf{4 t}$}

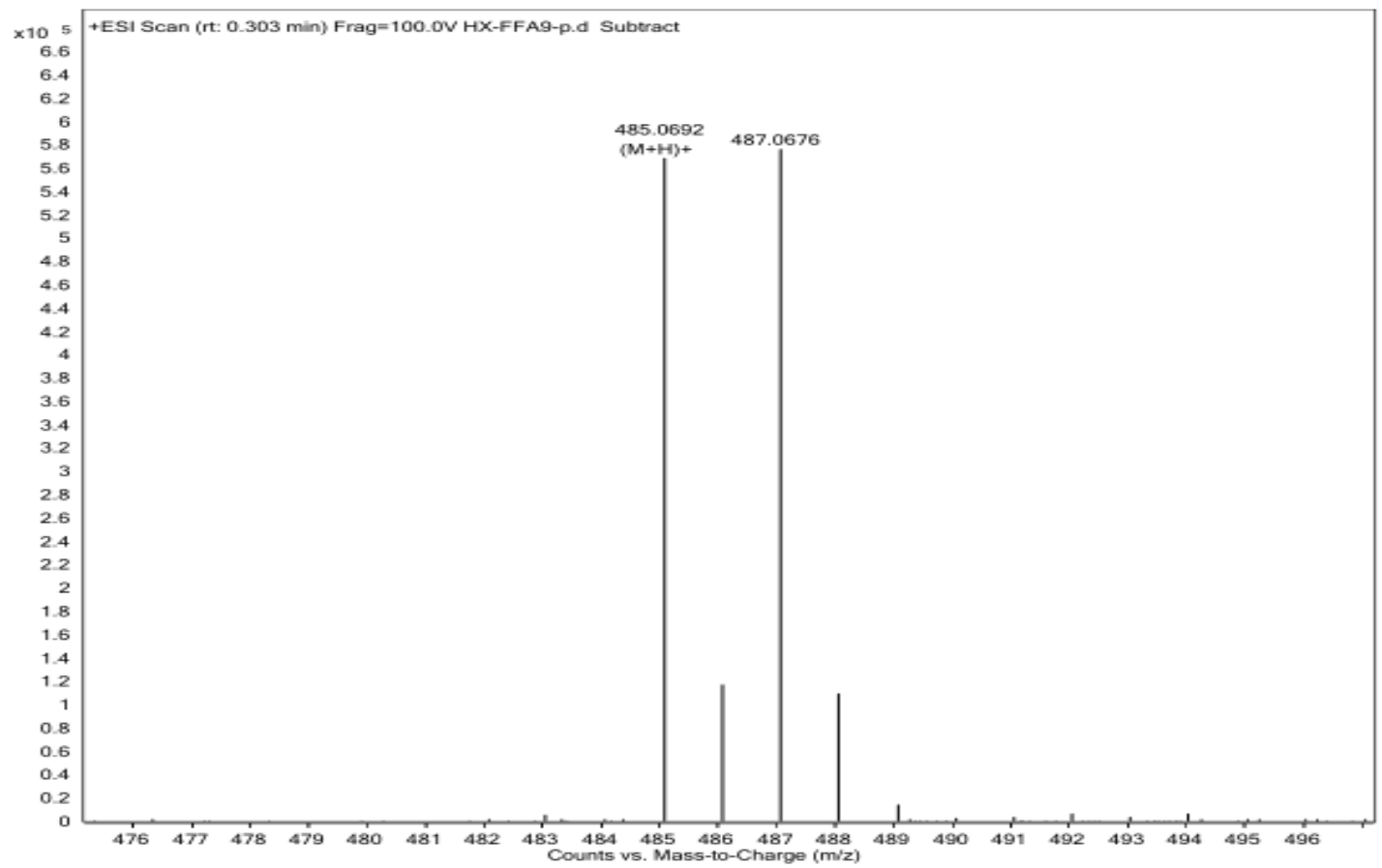


HRMS of $4 \mathbf{u}$

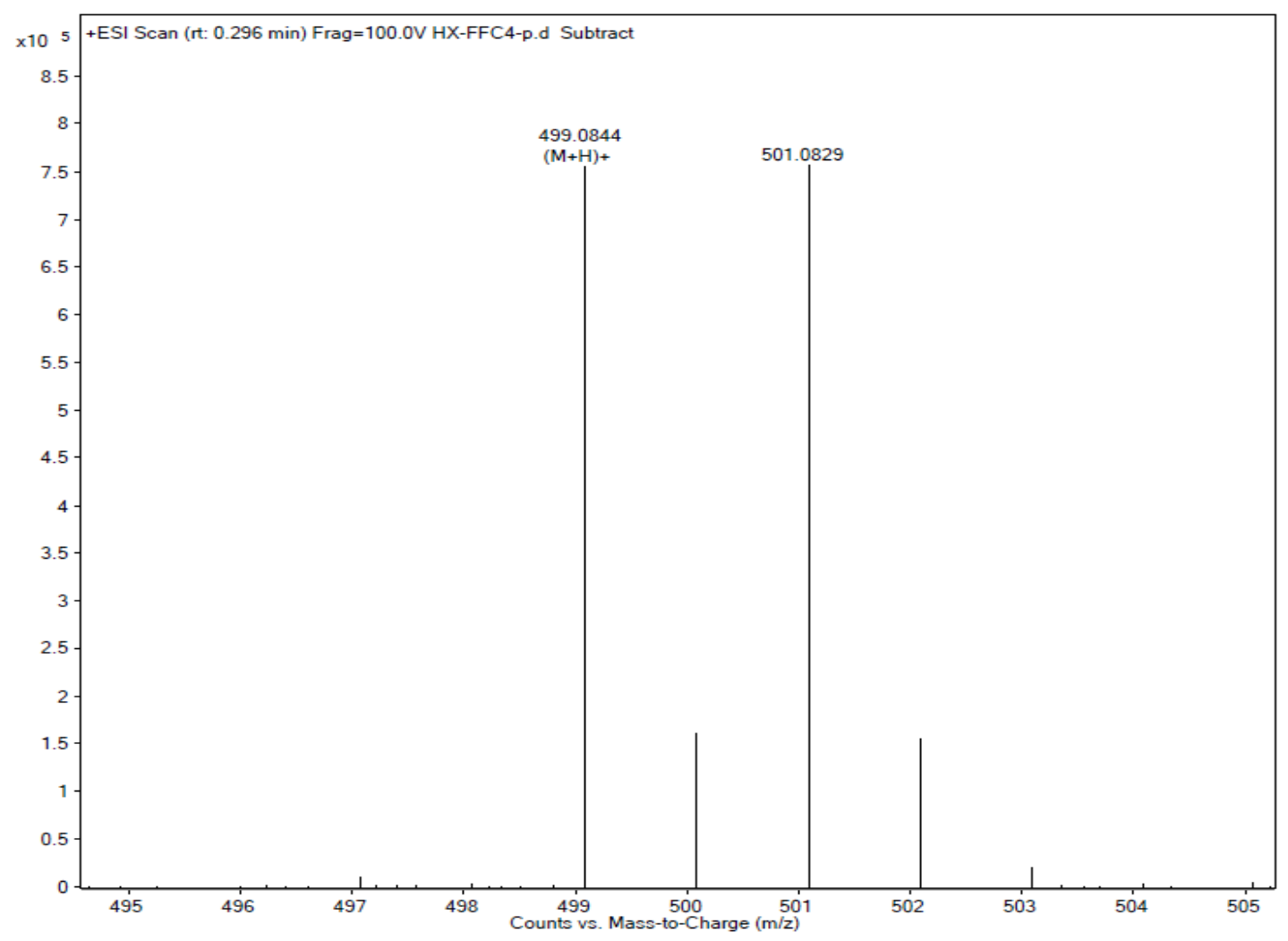

HRMS of $4 v$

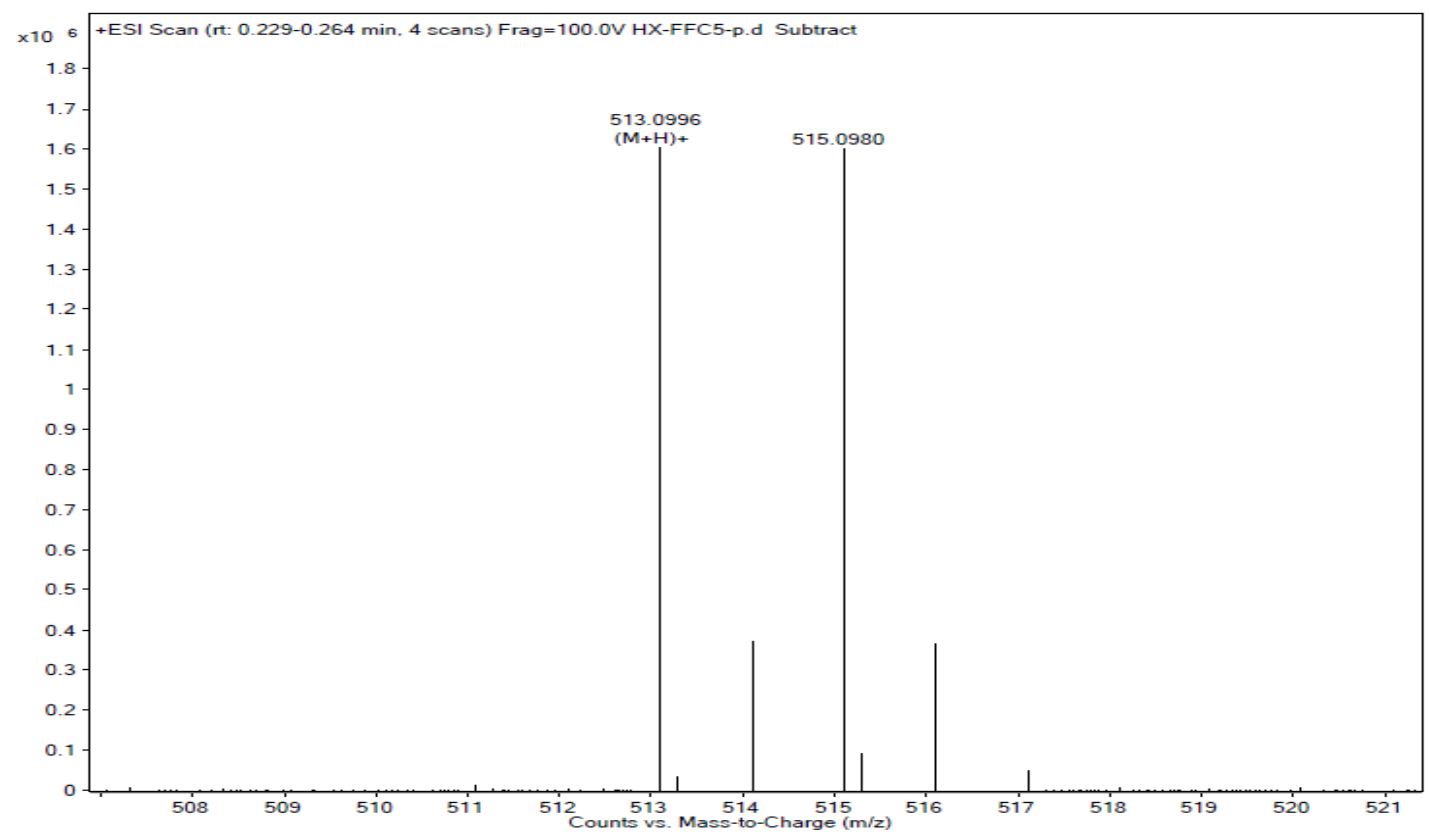




\section{HPLC Spectra of All Target Compounds.}

HPLC conditions:

Column: Wondasil C18 $(4.6 \times 250 \mathrm{~mm}, 5 \mu \mathrm{m})$

Wave length: $254 \mathrm{~nm}$

Temperature: $35^{\circ} \mathrm{C}$

Flow rate: $1 \mathrm{~mL} / \mathrm{min}$

Injection: $10 \mu \mathrm{L}$

Mobile phase: A phase: buffer ( $0.1 \%$ triethylamine, phosphoric acid to adjust $\mathrm{pH}$ to 7.0$)$

$$
\text { B phase: } \mathrm{MeOH}
$$

Gradient elution: 0-10 $\min (\mathrm{MeOH}$ 60-80\%), 10-20 $\min (\mathrm{MeOH} 80 \%), 20-20.01 \mathrm{~min}(\mathrm{MeOH} 80-$ 60\%), 20.01-30 $\min (\mathrm{MeOH} 60 \%)$.

HPLC spectrogram for compound $\mathbf{4 b}$

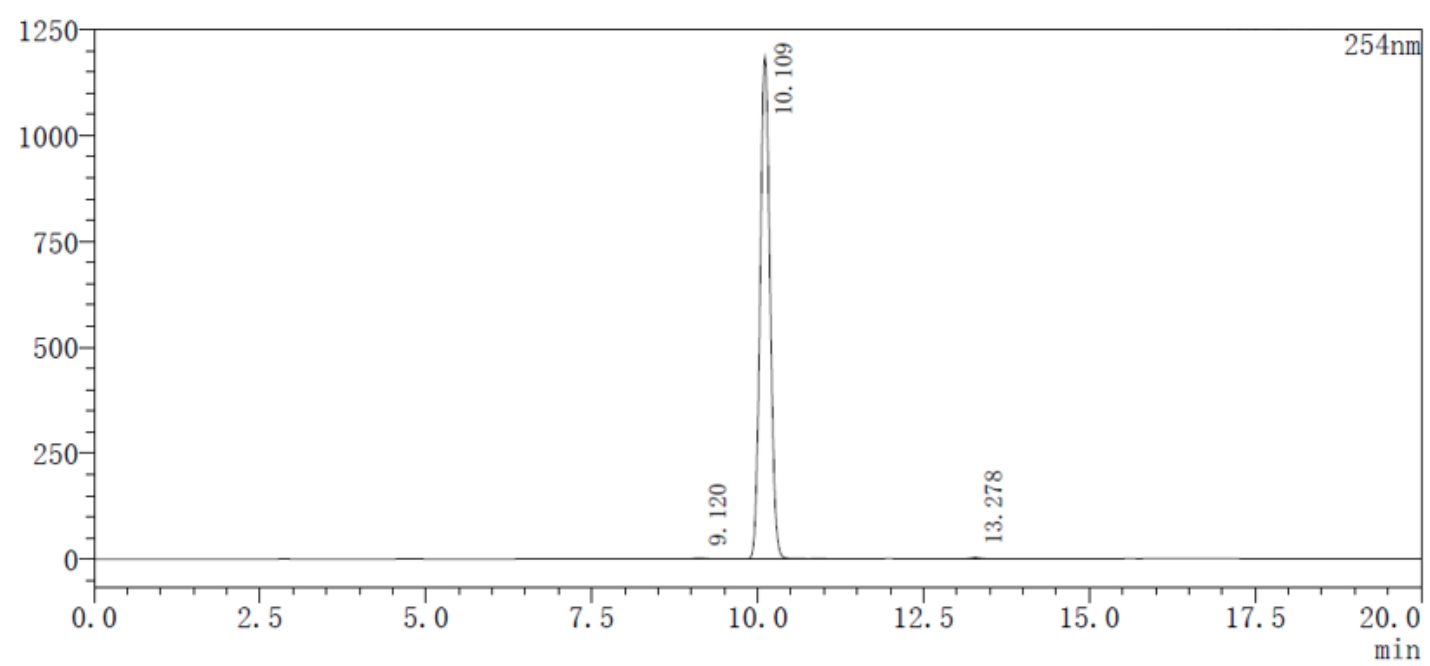

HPLC spectrogram for compound $\mathbf{4 a}$

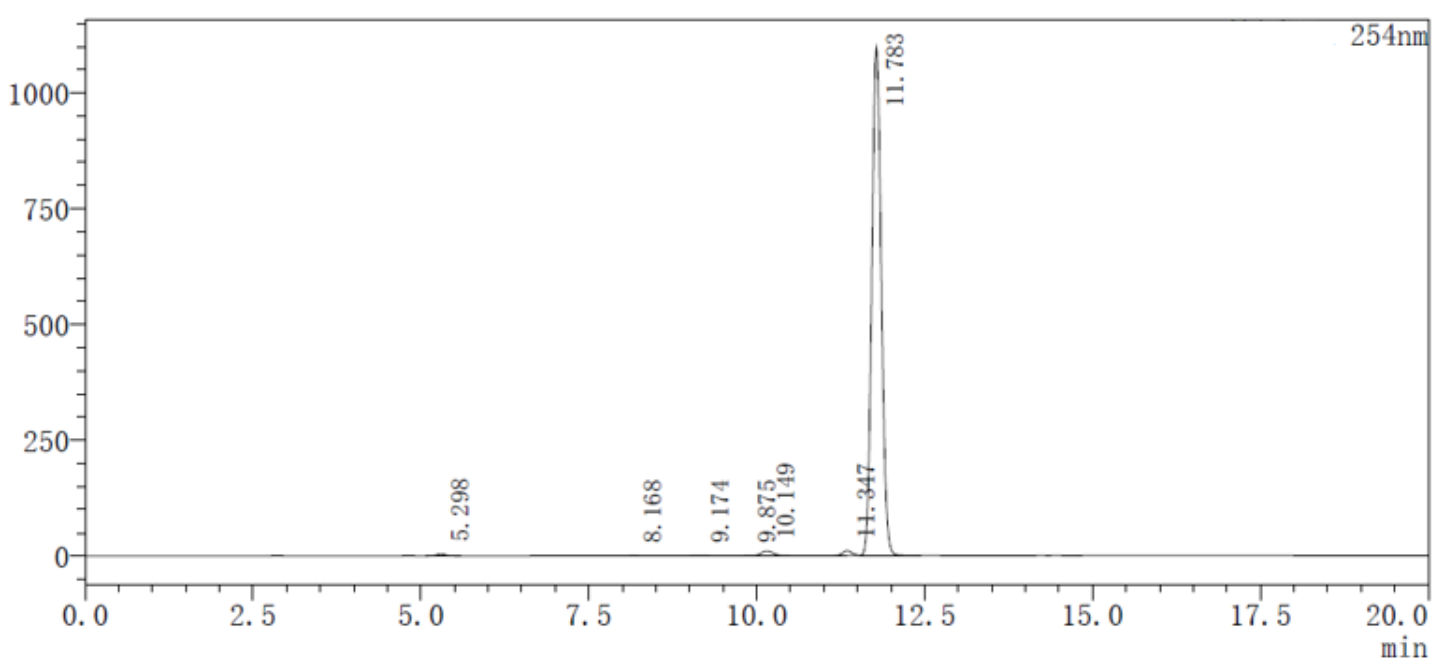


HPLC spectrogram for compound $\mathbf{4 c}$

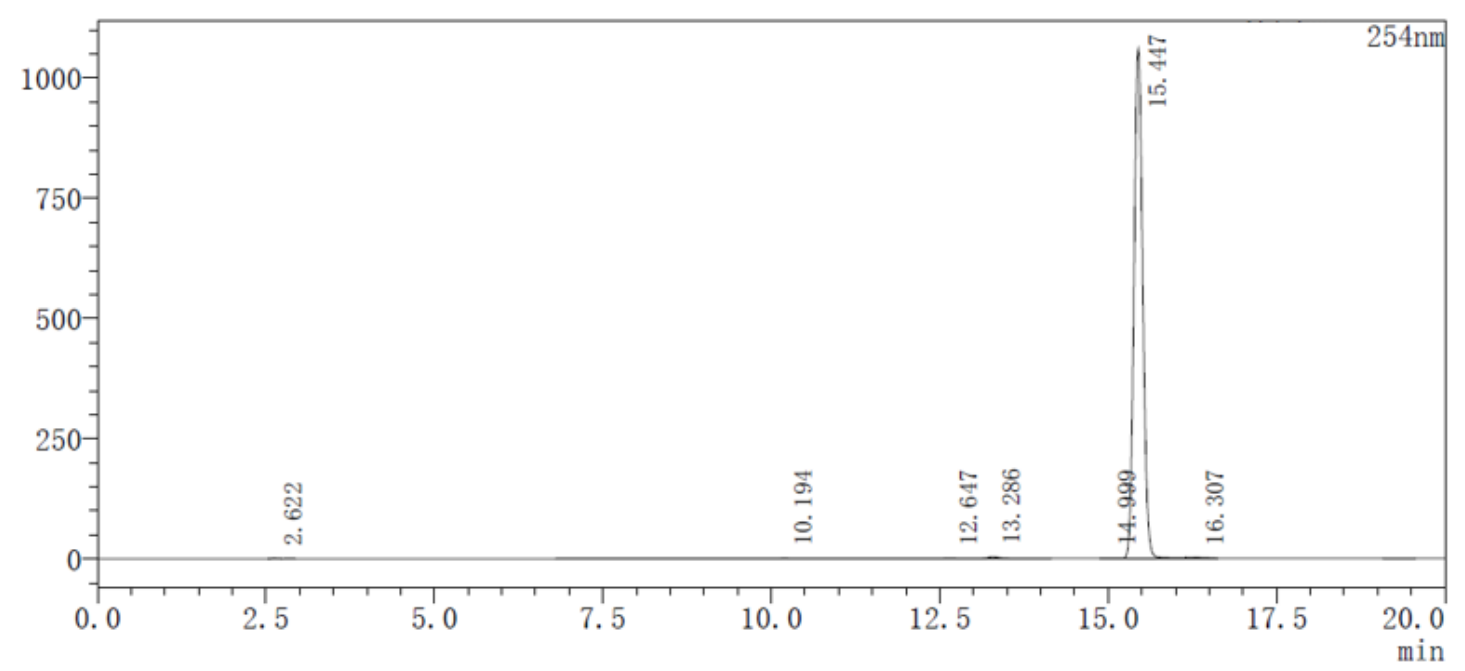

HPLC spectrogram for compound $\mathbf{4 d}$

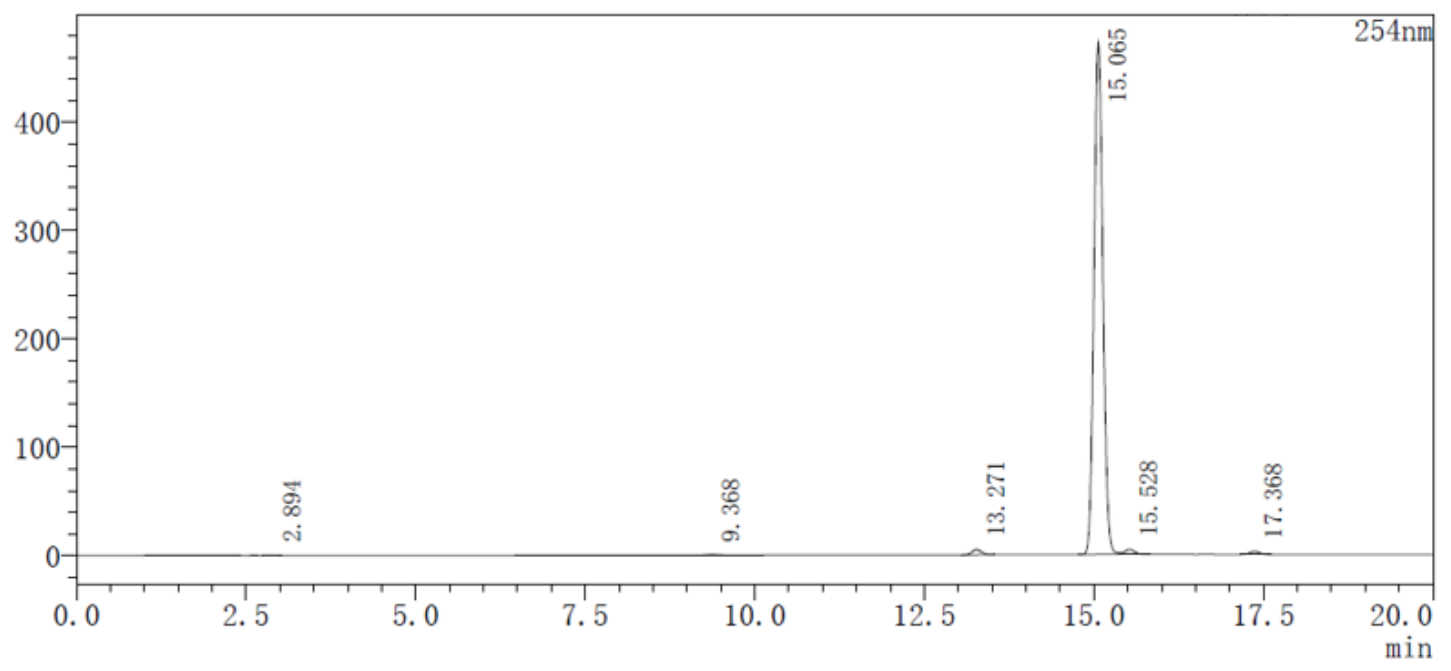

HPLC spectrogram for compound $\mathbf{4 e}$

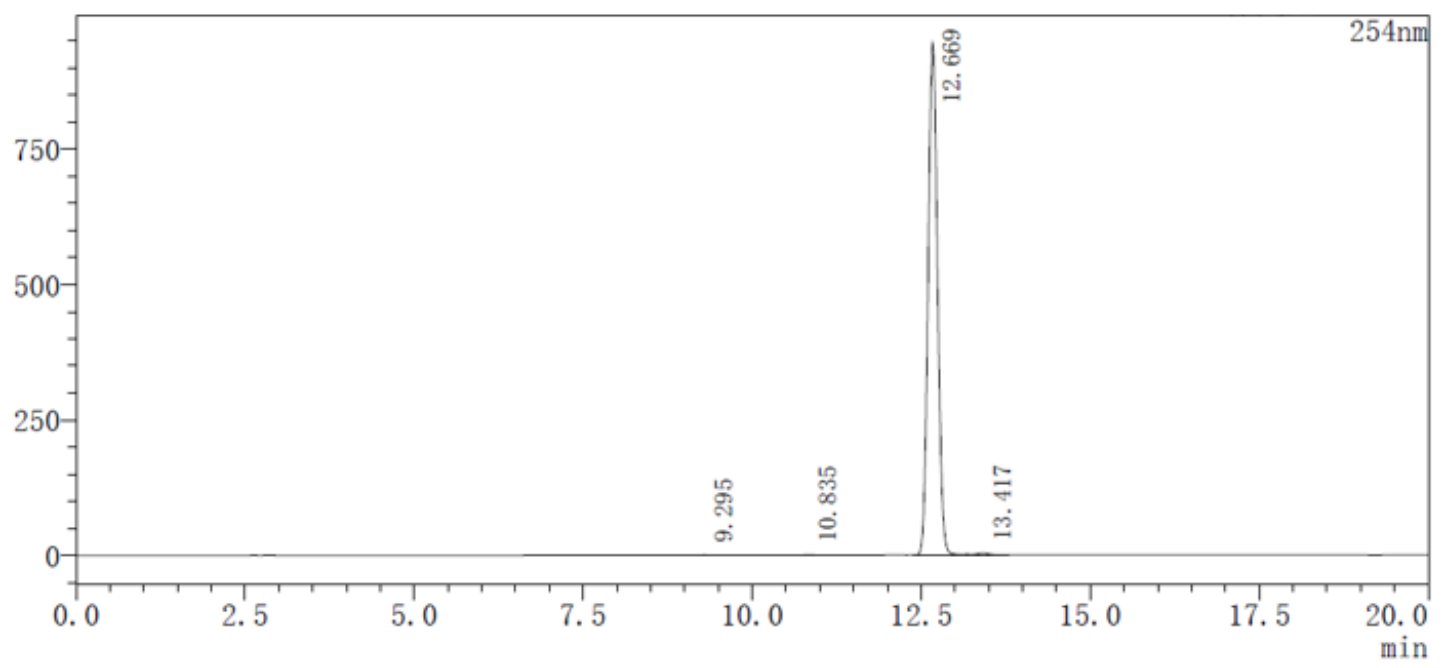


HPLC spectrogram for compound $\mathbf{4 f}$

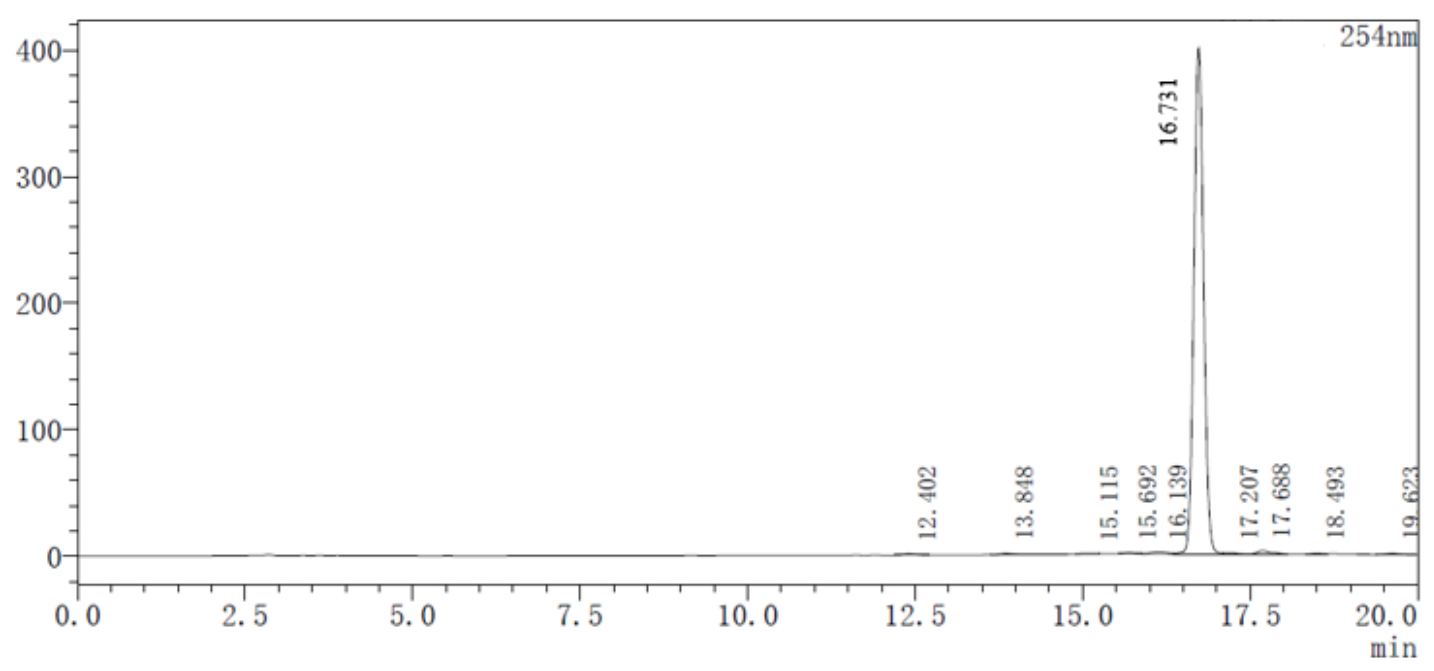

HPLC spectrogram for compound $\mathbf{4 g}$

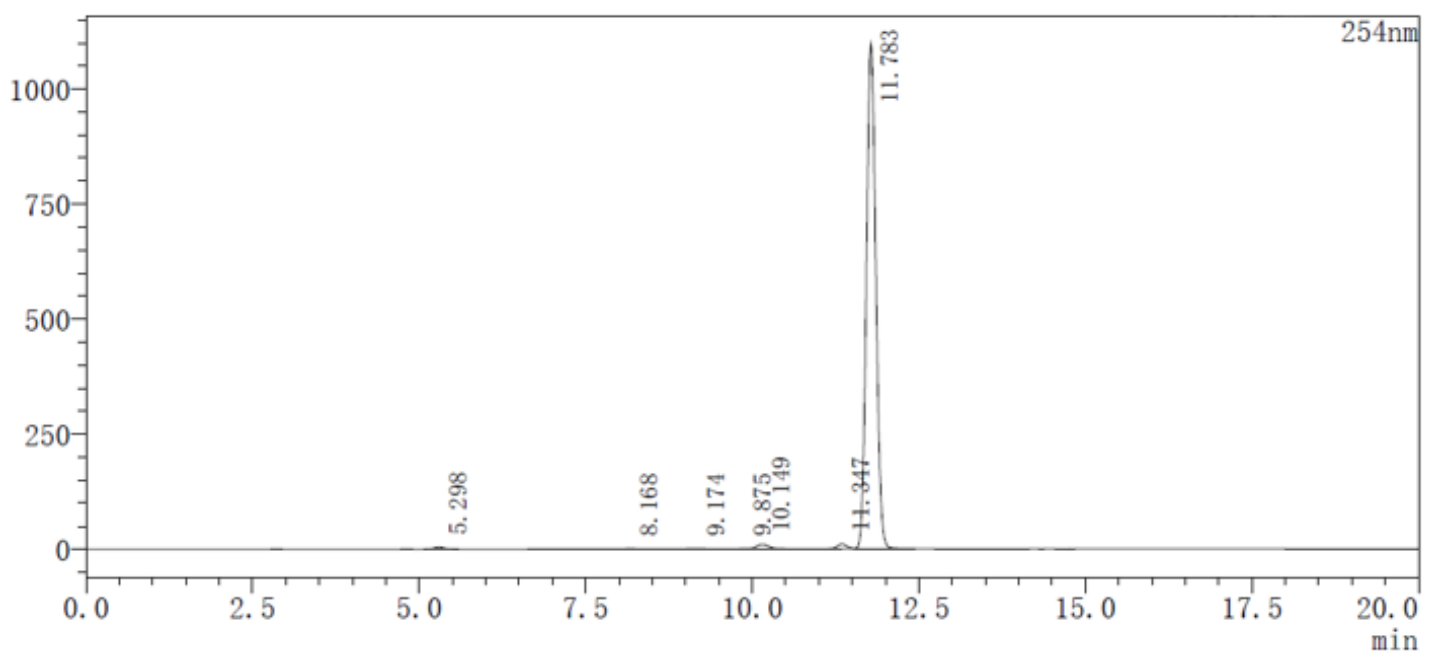

HPLC spectrogram for compound $\mathbf{4 h}$

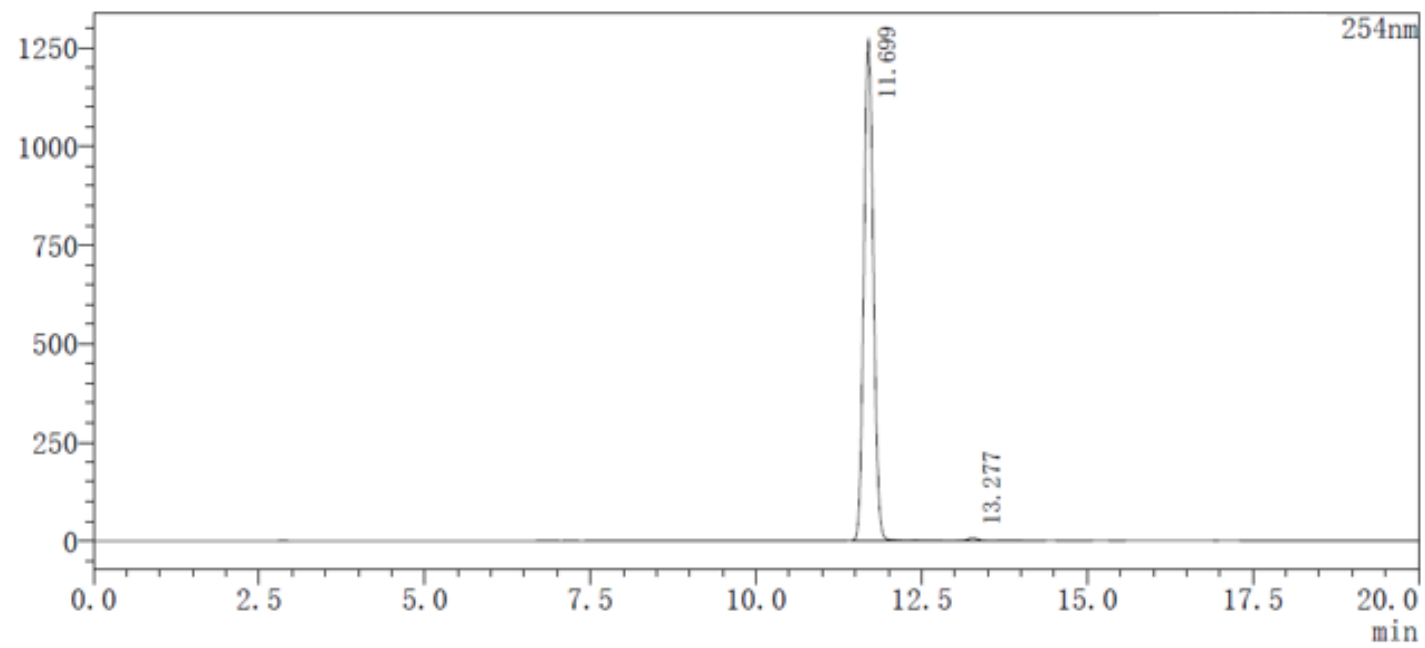


HPLC spectrogram for compound $\mathbf{4 i}$

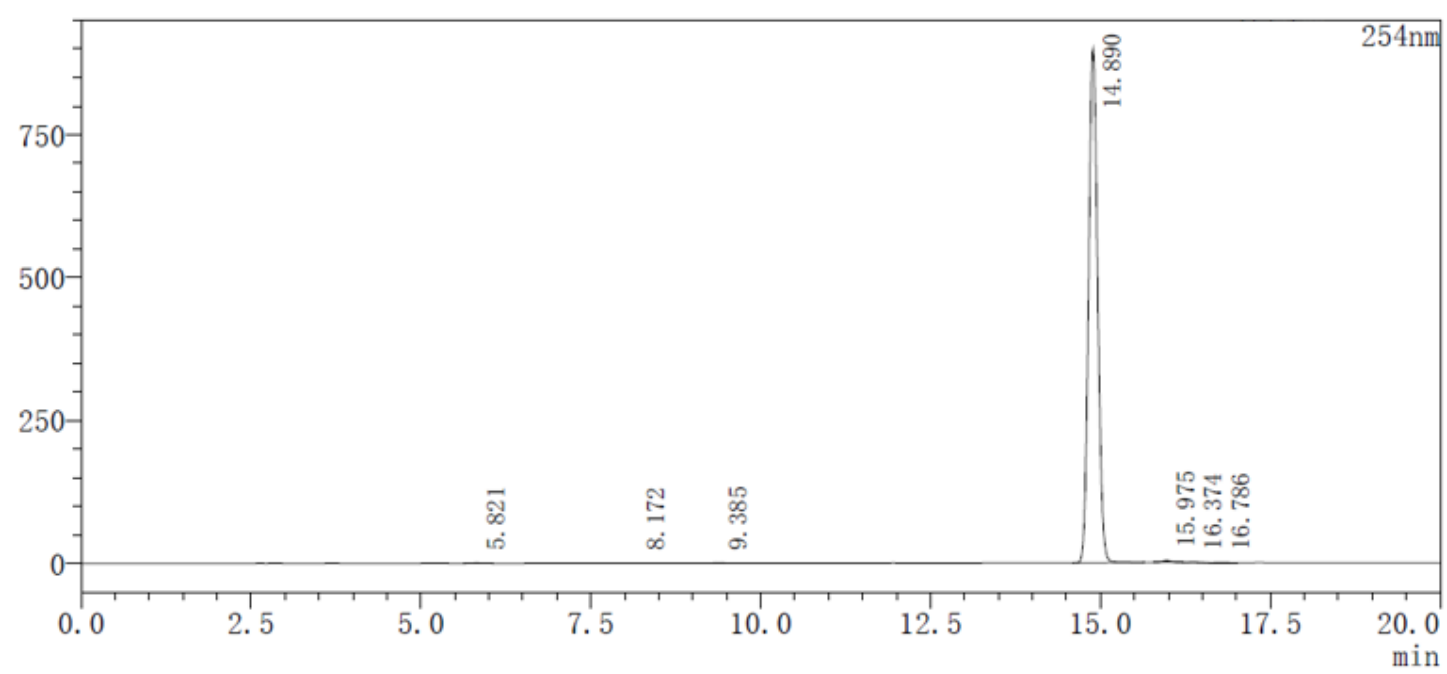

HPLC spectrogram for compound $\mathbf{4} \mathbf{j}$

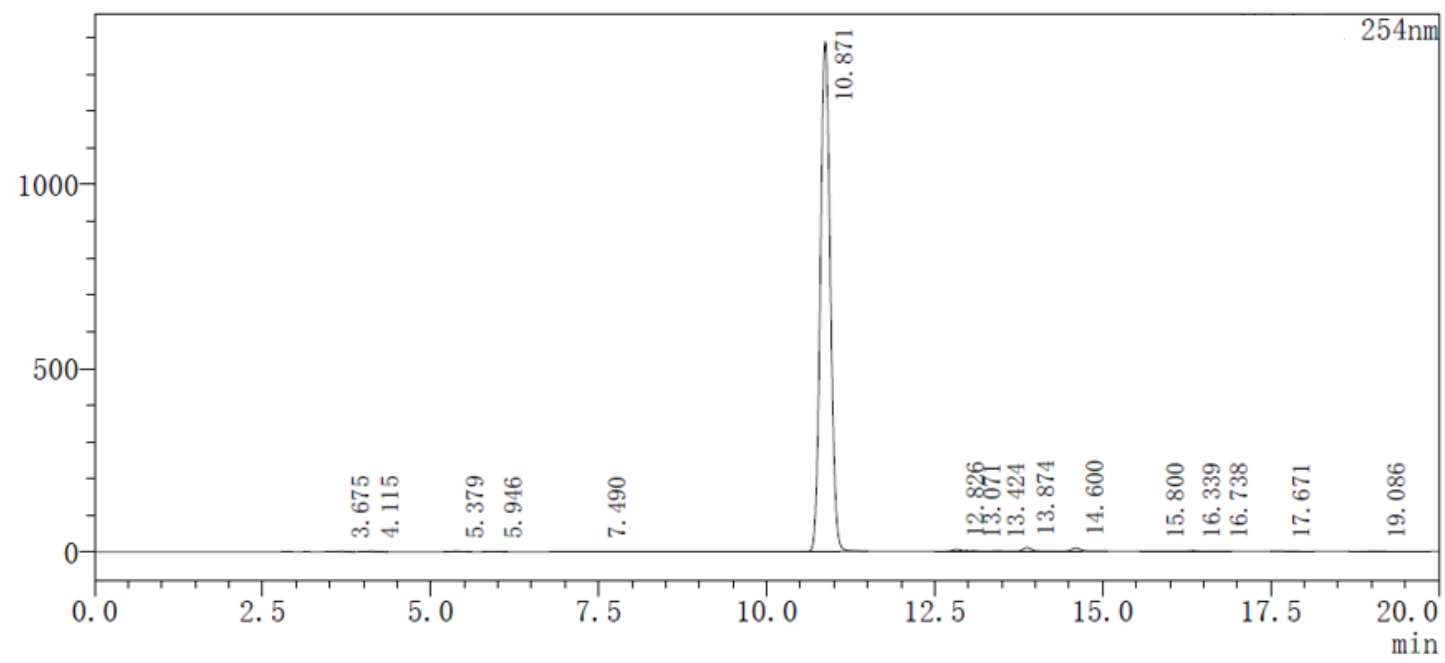

HPLC spectrogram for compound $\mathbf{4 k}$

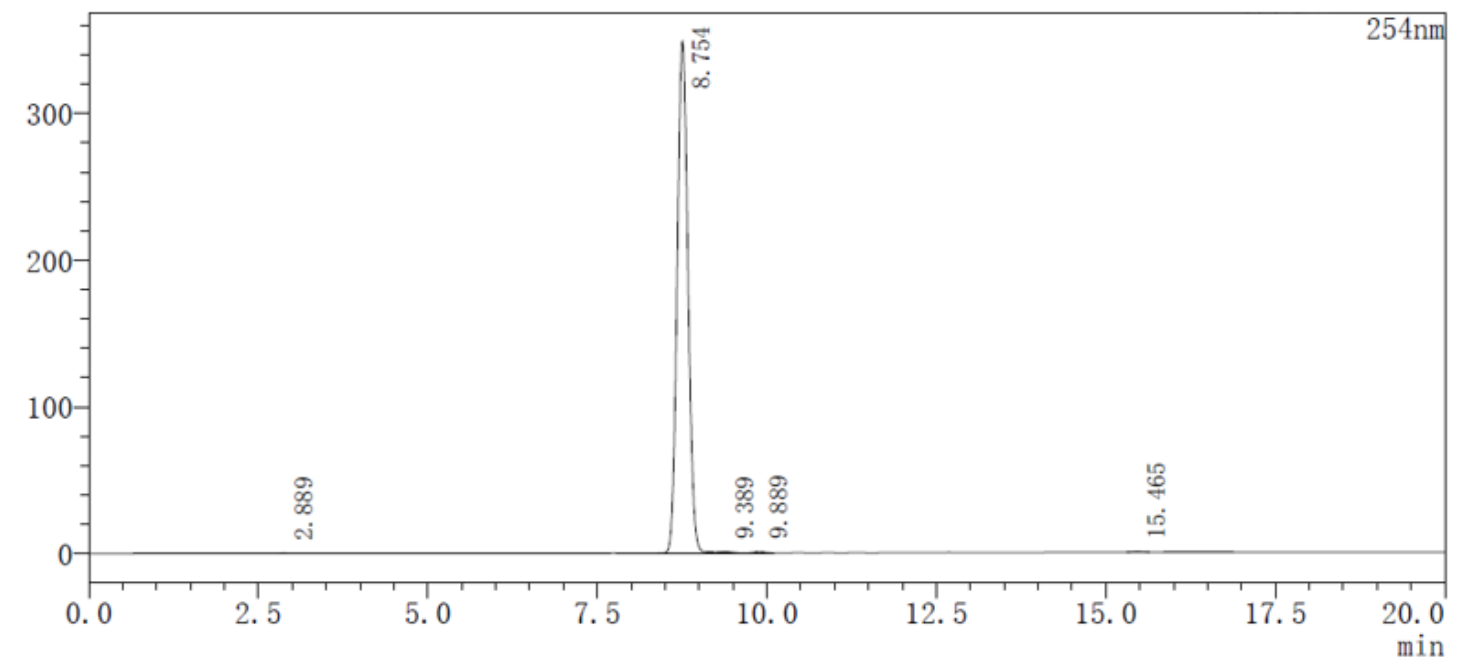


HPLC spectrogram for compound 41

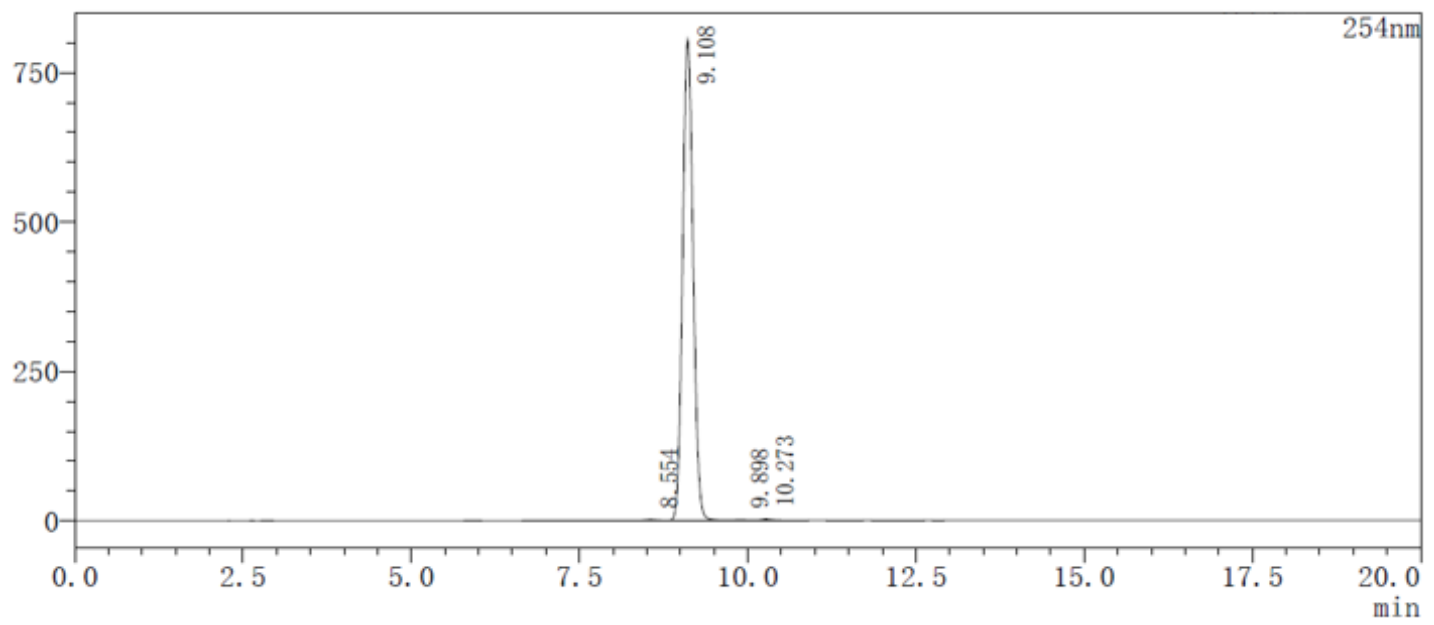

HPLC spectrogram for compound $\mathbf{4 m}$

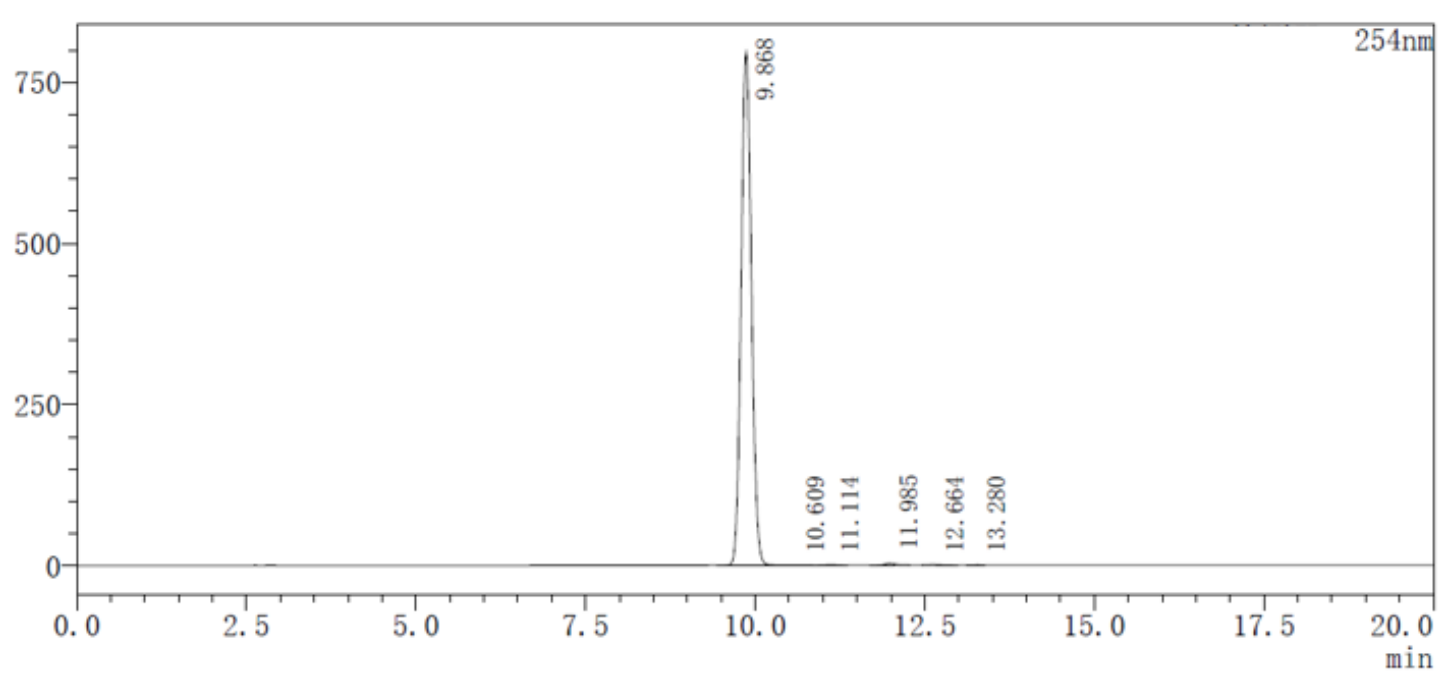

HPLC spectrogram for compound $\mathbf{4 n}$

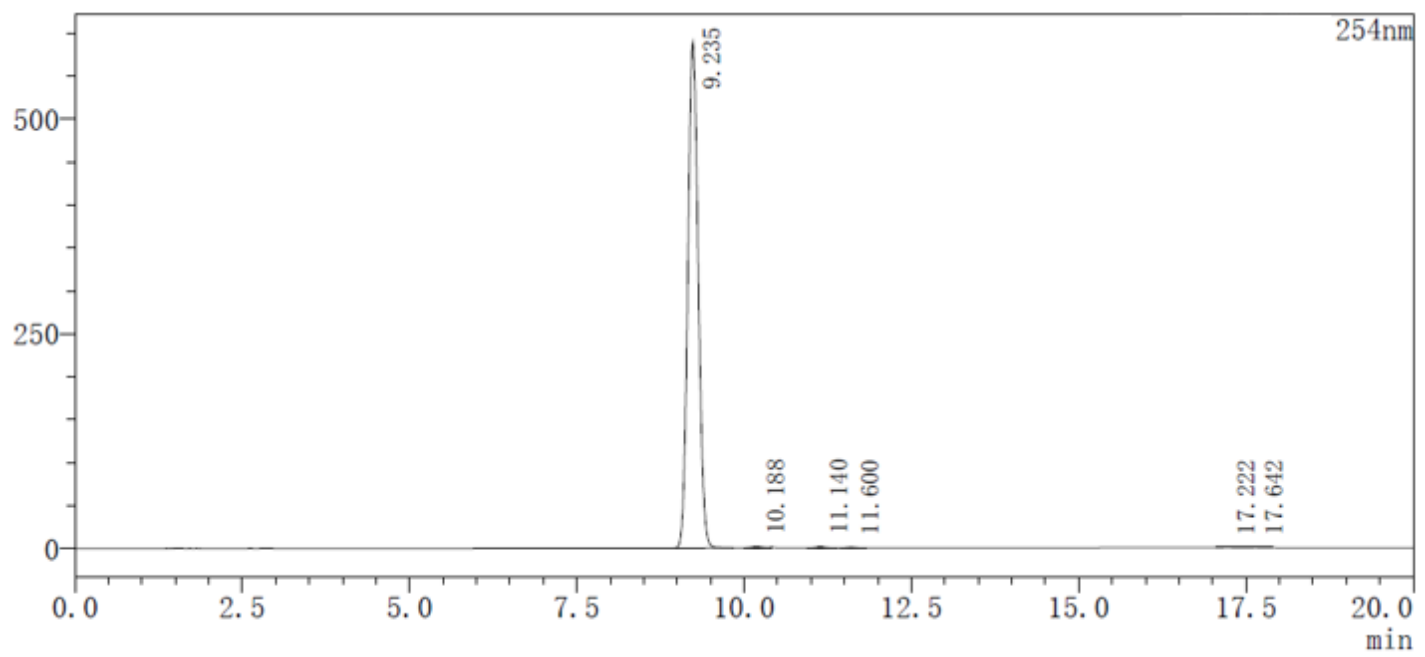


HPLC spectrogram for compound 40

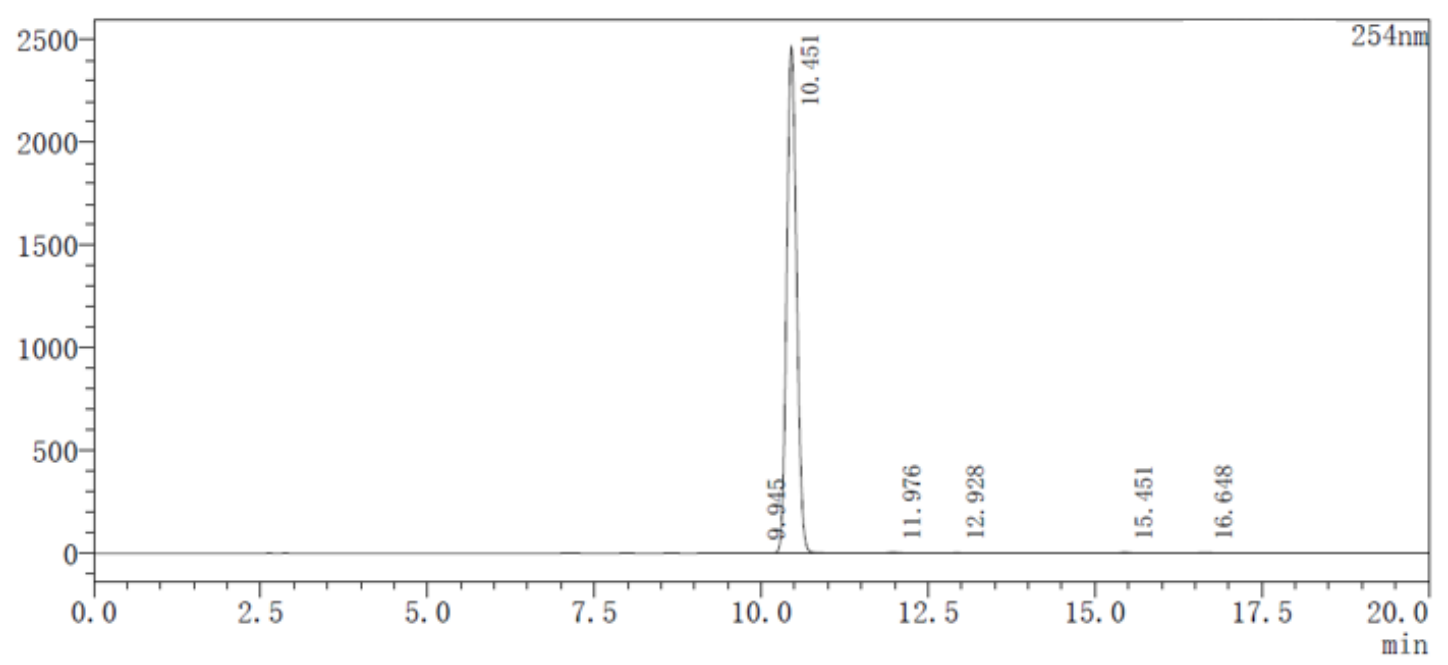

HPLC spectrogram for compound $\mathbf{4 p}$

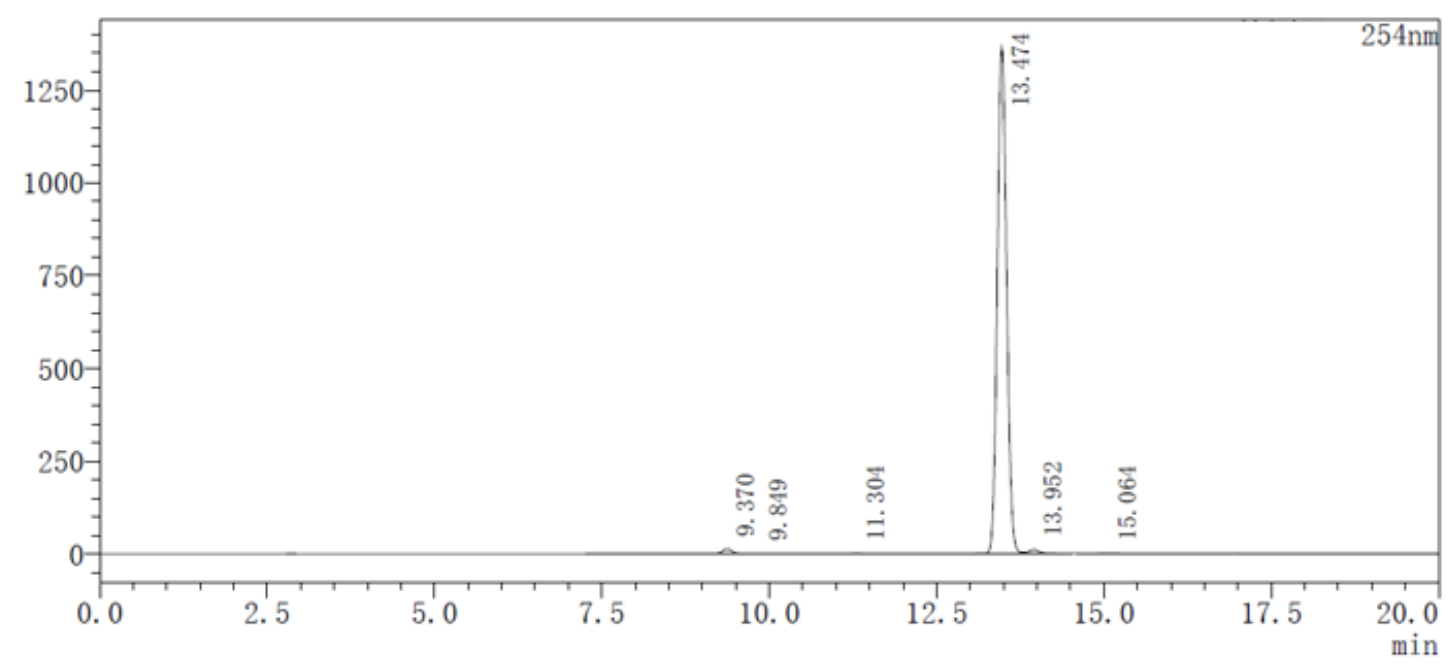

HPLC spectrogram for compound $\mathbf{4 q}$

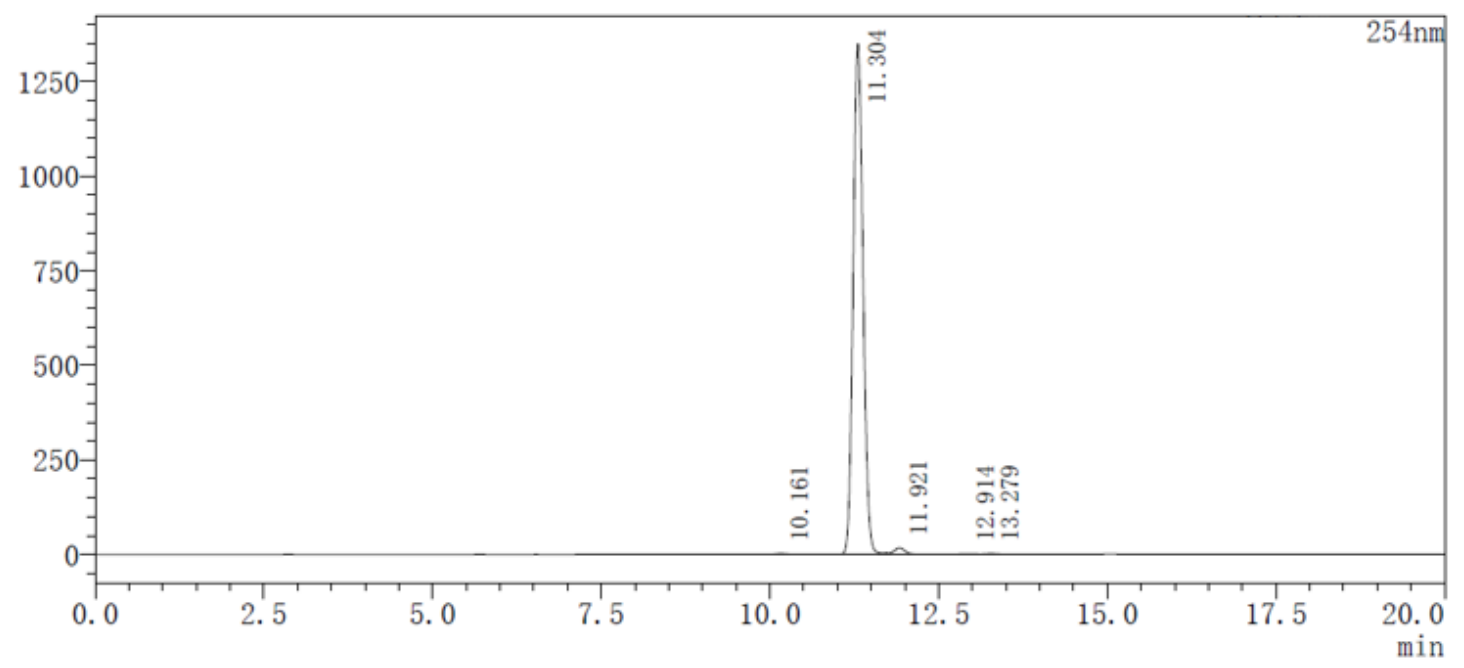


HPLC spectrogram for compound $\mathbf{4 r}$

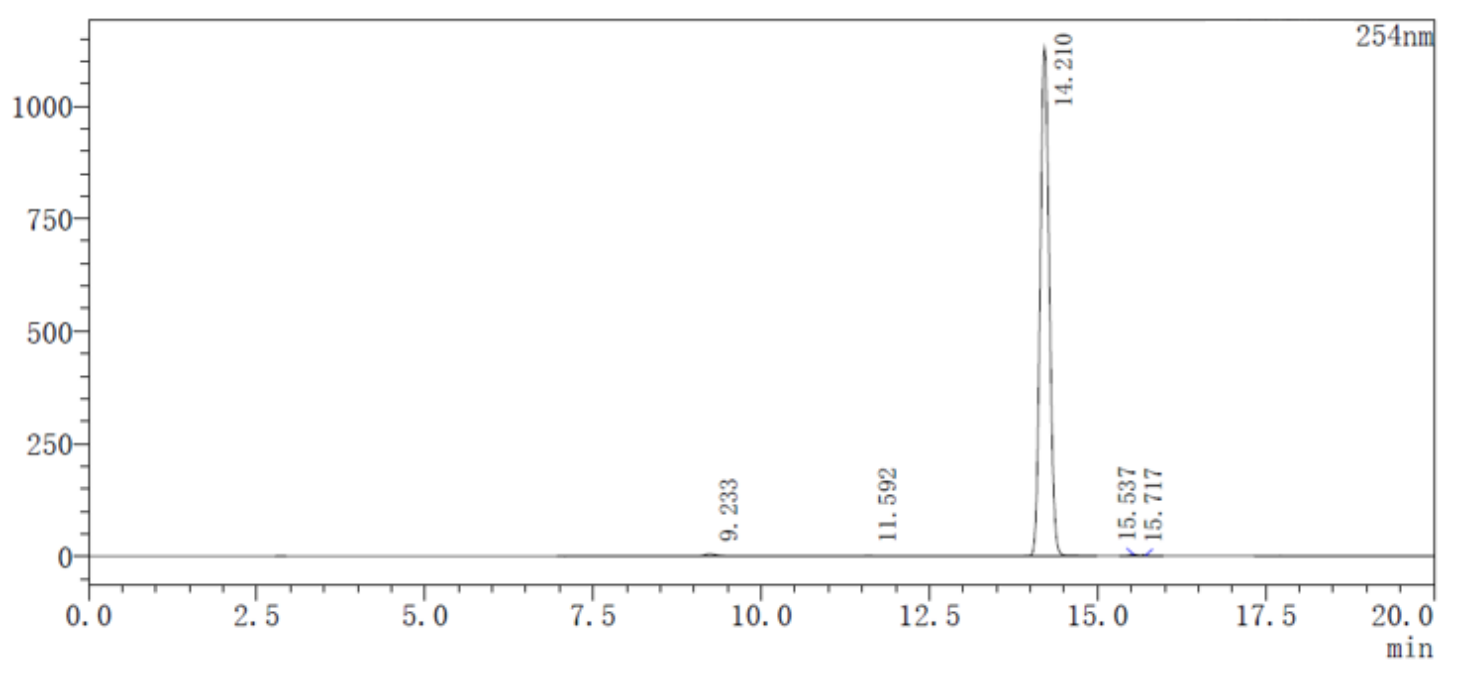

HPLC spectrogram for compound $\mathbf{4 s}$

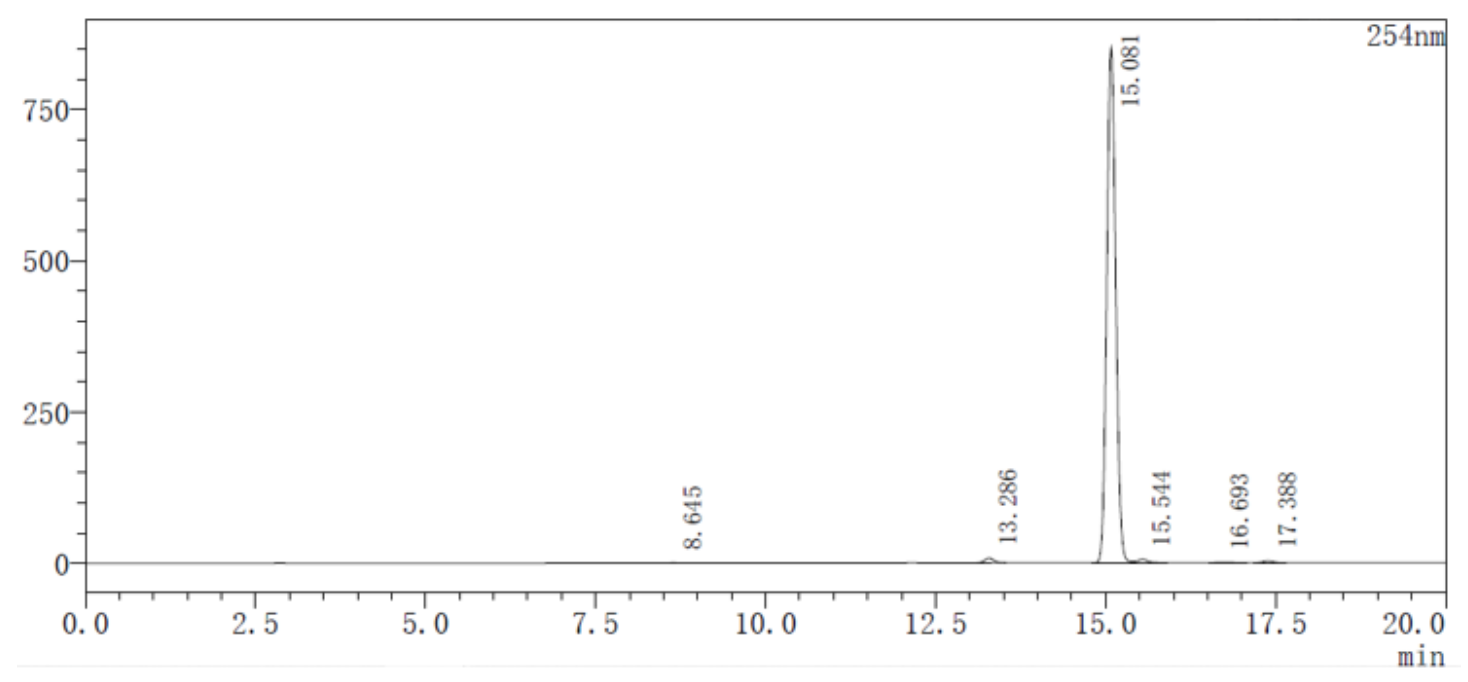

HPLC spectrogram for compound $\mathbf{4 t}$

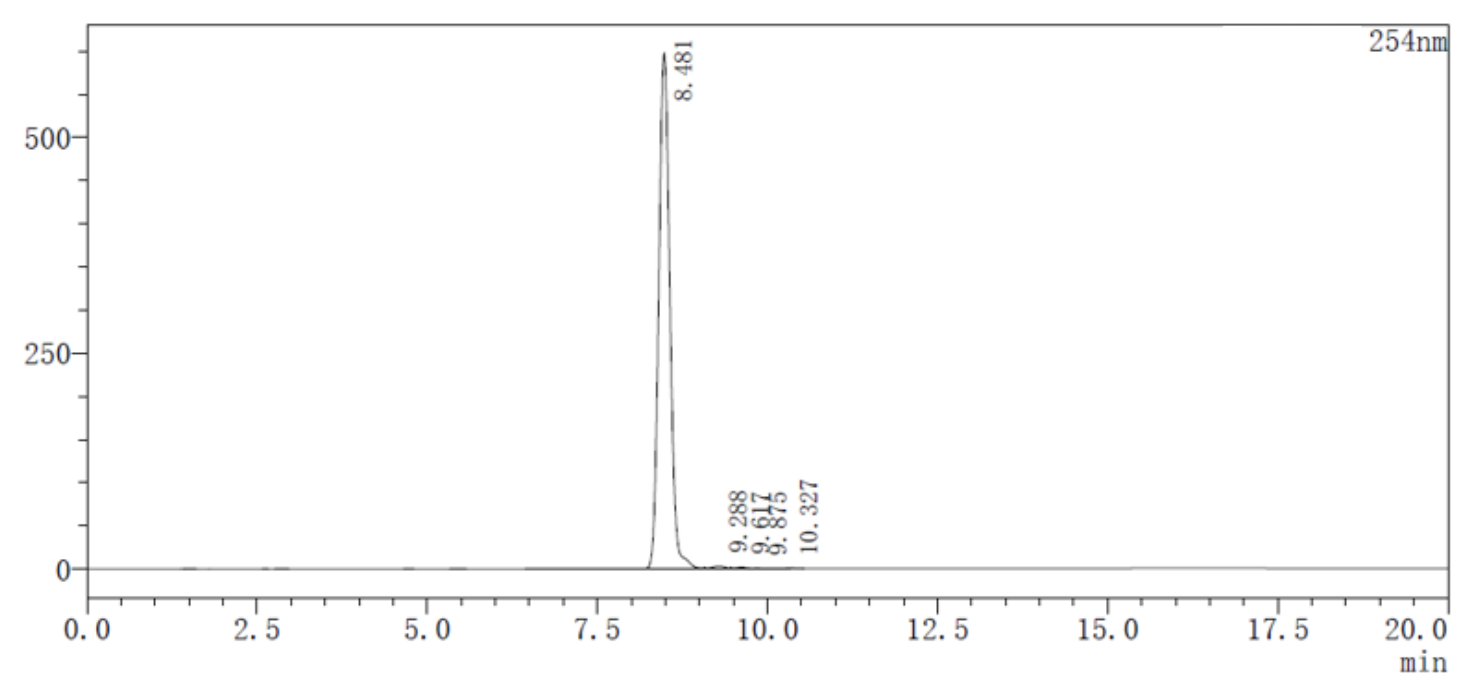


HPLC spectrogram for compound $\mathbf{4 u}$

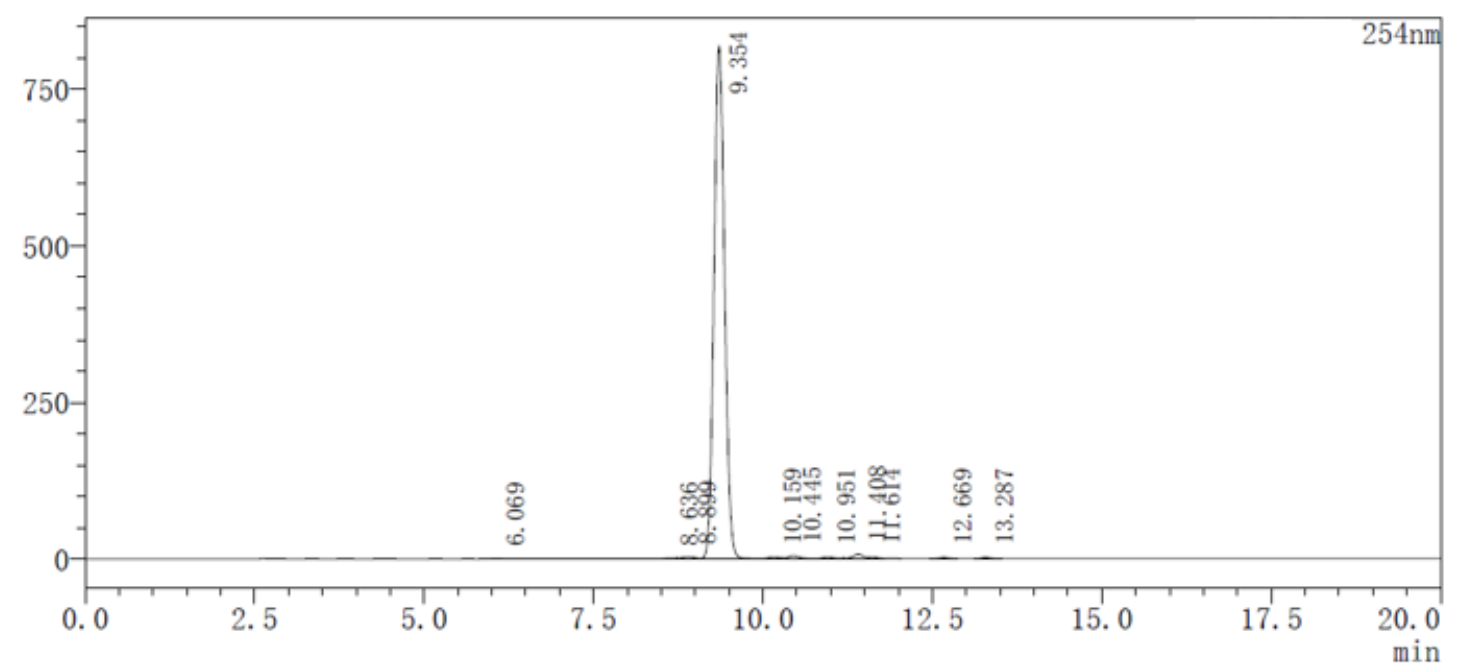

HPLC spectrogram for compound $\mathbf{4 v}$

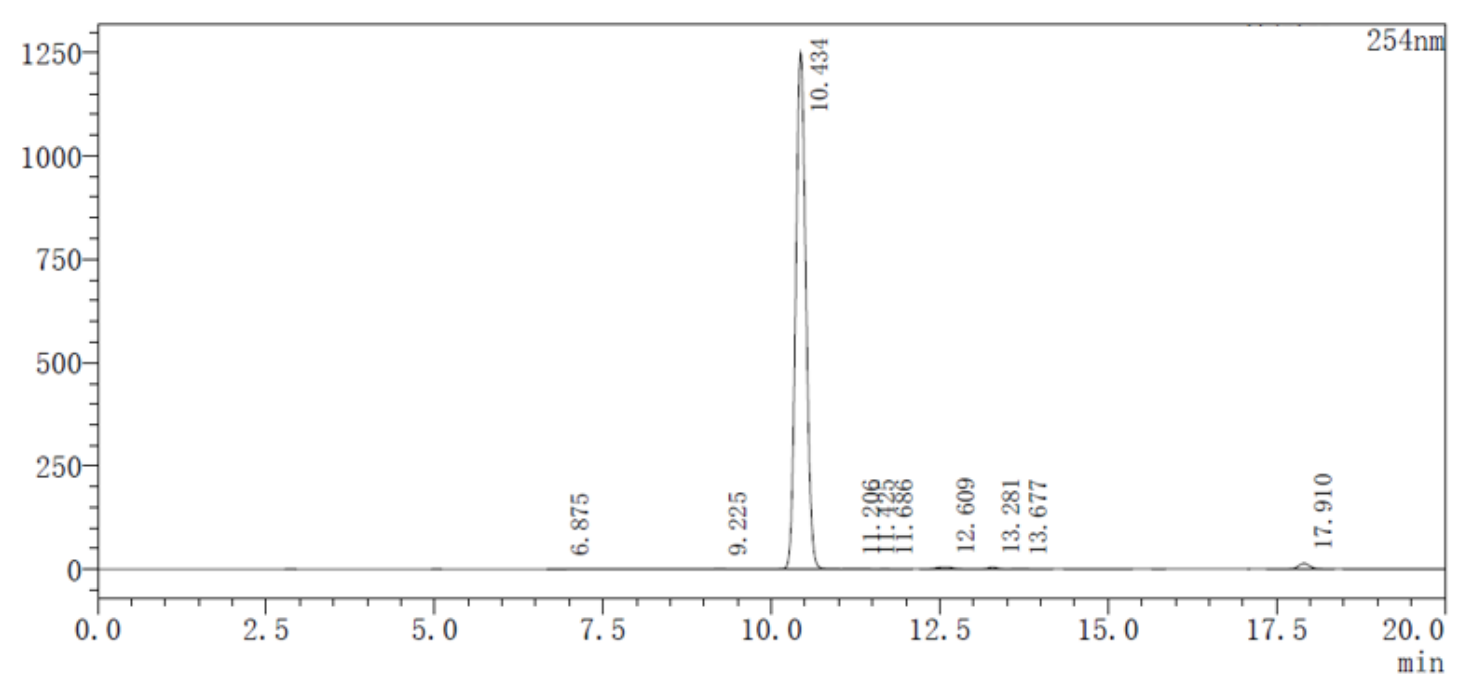

